ESAIM: M2AN 50 (2016) 1659-1697

DOI: $10.1051 / \mathrm{m} 2 \mathrm{an} / 2016003$
ESAIM: Mathematical Modelling and Numerical Analysis

www.esaim-m2an.org

\title{
FINITE ELEMENT HETEROGENEOUS MULTISCALE METHOD FOR NONLINEAR MONOTONE PARABOLIC HOMOGENIZATION PROBLEMS
}

\author{
Assyr Abdulle ${ }^{1}$ And Martin E. Huber ${ }^{1}$
}

\begin{abstract}
We propose a multiscale method based on a finite element heterogeneous multiscale method (in space) and the implicit Euler integrator (in time) to solve nonlinear monotone parabolic problems with multiple scales due to spatial heterogeneities varying rapidly at a microscopic scale. The multiscale method approximates the homogenized solution at computational cost independent of the small scale by performing numerical upscaling (coupling of macro and micro finite element methods). Taking into account the error due to time discretization as well as macro and micro spatial discretizations, the convergence of the method is proved in the general $L^{p}\left(W^{1, p}\right)$ setting. For $p=2$, optimal convergence rates in the $L^{2}\left(H^{1}\right)$ and $\mathcal{C}^{0}\left(L^{2}\right)$ norm are derived. Numerical experiments illustrate the theoretical error estimates and the applicability of the multiscale method to practical problems.
\end{abstract}

Mathematics Subject Classification. 65N30, 65M60, 74Q10, 74D10.

Received February 9, 2015. Revised November 20, 2015. Accepted January 6, 2016.

\section{INTRODUCTION}

In this article, we propose a numerical method to solve nonlinear monotone parabolic multiscale problems

$$
\begin{gathered}
\partial_{t} u^{\varepsilon}(x, t)-\operatorname{div}\left(\mathcal{A}^{\varepsilon}\left(x, \nabla u^{\varepsilon}(x, t)\right)\right)=f(x) \text { in } \Omega \times(0, T), \\
u^{\varepsilon}(x, t)=0 \text { on } \partial \Omega \times(0, T), \quad u^{\varepsilon}(x, 0)=g(x) \text { in } \Omega,
\end{gathered}
$$

with given source $f$, initial condition $g$ and maps $\mathcal{A}^{\varepsilon}: \Omega \times \mathbb{R}^{d} \rightarrow \mathbb{R}^{d}$ (indexed by $\varepsilon$ ) on a convex polygonal domain $\Omega \subset \mathbb{R}^{d}, d \leq 3$, and a finite time interval $(0, T)$. The variable $\varepsilon>0$ represents a small scale in the problem, at which the maps $\mathcal{A}^{\varepsilon}(\cdot, \xi)$ highly oscillate. Note that our results can be straightforwardly extended to problems (1.1) with time dependent sources $f(x, t)$ and different boundary conditions.

The model problem (1.1) is studied in the $L^{p}\left(W^{1, p}\right)$ setting for $p \in \mathbb{R}$ with $1<p<\infty$ and $p>2 d /(d+2)$. We assume that the maps $\mathcal{A}^{\varepsilon}$ satisfy the following conditions uniformly in $\varepsilon>0$

$\left(\mathcal{A}_{0}\right)$ there is some $C_{0} \geq 0$ such that $\left|\mathcal{A}^{\varepsilon}(x, 0)\right| \leq C_{0}$ for almost every (a.e.) $x \in \Omega$;

\footnotetext{
Keywords and phrases. Nonlinear monotone parabolic problem, multiple scales, heterogeneous multiscale method, finite elements, implicit Euler, fully discrete error, resonance error.

1 ANMC, Mathematics Section, École Polytechnique Fédérale de Lausanne, 1015 Lausanne, Switzerland.

Assyr.Abdulle@epfl.ch; Martin.Huber@epfl.ch
} 
$\left(\mathcal{A}_{1}\right)$ there exist $\kappa_{1} \geq 0, L>0$ and $0<\alpha \leq \min \{p-1,1\}$ such that

$$
\left|\mathcal{A}^{\varepsilon}\left(x, \xi_{1}\right)-\mathcal{A}^{\varepsilon}\left(x, \xi_{2}\right)\right| \leq L\left(\kappa_{1}+\left|\xi_{1}\right|+\left|\xi_{2}\right|\right)^{p-1-\alpha}\left|\xi_{1}-\xi_{2}\right|^{\alpha}, \quad \forall \xi_{1}, \xi_{2} \in \mathbb{R}^{d} \text {, a.e. } x \in \Omega ;
$$

$\left(\mathcal{A}_{2}\right)$ there exist $\kappa_{2} \geq 0, \lambda>0$ and $\max \{2, p\} \leq \beta<\infty$ such that

$$
\left(\mathcal{A}^{\varepsilon}\left(x, \xi_{1}\right)-\mathcal{A}^{\varepsilon}\left(x, \xi_{2}\right)\right) \cdot\left(\xi_{1}-\xi_{2}\right) \geq \lambda\left(\kappa_{2}+\left|\xi_{1}\right|+\left|\xi_{2}\right|\right)^{p-\beta}\left|\xi_{1}-\xi_{2}\right|^{\beta}, \quad \forall \xi_{1}, \xi_{2} \in \mathbb{R}^{d}, \text { a.e. } x \in \Omega,
$$

see Examples 1-3 in Section 2 for illustration. Those are the most general hypotheses for the maps $\mathcal{A}^{\varepsilon}$ under which homogenization for (1.1) can be established, see [15,18,42]. Many physical processes can be modeled by parabolic partial differential equations (PDEs) of the form (1.1), e.g., non-Newtonian fluids, ferromagnetic materials or composites with nonlinear materials, see [12,41].

Using standard numerical methods, like the finite element method (FEM), to discretize the problem (1.1) in space leads to high computational cost as the small scale $\varepsilon$ of the spatial heterogeneities of $\mathcal{A}^{\varepsilon}$ has to be resolved. Thus, to efficiently approximate the solution of (1.1) at the scale of interest, effective models for (1.1) are needed. Homogenization theory, see [13,37], is the usual framework used to study the solutions $u^{\varepsilon}$ to (1.1) in the limit $\varepsilon \rightarrow 0$ and aims at characterizing a limiting function $u^{0}$ as the solution of a homogenized (or effective) equation. The upscaling of (1.1) has been studied by Pankov and Svanstedt in [42] and [44], respectively, using the notion of parabolic $G$-convergence (extending the work by Tartar, see [46] and ([47], Chap. 11), and Chiado'Piat et al., see [15], to parabolic problems). In particular, the homogenized equation (with solution $u^{0}$ ) is again of the same type as (1.1) with $\mathcal{A}^{\varepsilon}$ replaced by the homogenized map $\mathcal{A}^{0}$ for which the small scales are averaged out.

For linear homogenization PDEs, a broad literature about multiscale methods exists nowadays, see $[2,4]$ (elliptic problems), [8,39] (parabolic problems) and the references therein. Numerical homogenization methods for nonlinear problems are however less numerous, e.g., see [9] for multiscale methods for PDEs with a nonmonotone nonlinearity (with respect to the solution $u^{\varepsilon}$ ). For parabolic multiscale PDEs (1.1) with monotone nonlinearities (with respect to the gradient $\nabla u^{\varepsilon}$ ), Svanstedt et al. proposed in [45] a numerical method for periodically oscillating (in space and time) maps $\mathcal{A}^{\varepsilon}$ based on an augmented Lagrangian method (for $p \geq 2$ and $0<\alpha \leq 1$, $\beta=p$ in $\left.\left(\mathcal{A}_{1-2}\right)\right)$. In [26], Efendiev et al. applied a generalized multiscale finite element method (MsFEM) developed in $[25,27]$ for elliptic monotone problems - to problem (1.1) with stochastic heterogeneities (for $p \geq 2$ and $0<\alpha \leq 1, \beta=p$ in $\left.\left(\mathcal{A}_{1-2}\right)\right)$.

In the numerical methods proposed in $[25,26]$ it is assumed that local problems (formulated on the elements of a coarse mesh) are solved exactly. In turn, the convergence analysis in the spatial $W^{1, p}$ norm does not take into account the variational crimes due to numerical quadrature required to assemble the stiffness matrix nor the numerical approximation of the local problems which are both necessary for a practical implementation. Additionally, the convergence results for the parabolic problems studied in [26] neglect the effect of the time discretization error. We note that in both $[26,45]$ convergence of the numerical solution to the homogenized solution is shown without deriving explicit convergence rates.

For completeness we briefly review numerical methods for elliptic monotone multiscale PDEs. In [33], a sparse tensor FEM based on ideas developed in [34] has been analyzed (for $p \geq 2$ and $\alpha=1, \beta=p$ in $\left(\mathcal{A}_{1-2}\right)$ ). Related to the heterogeneous multiscale method (HMM), the framework used in this paper, we mention [32] and [29]. In [32], an a posteriori error estimate has been obtained for elliptic monotone problems (for $p=2$ and $\alpha=1, \beta=2$ in $\left(\mathcal{A}_{1-2}\right)$ ), but no a priori convergence rates have been derived. In [29] numerical homogenization methods (FE-HMM and MsFEM) for a class of elliptic monotone PDEs (associated to minimization problems) have been studied and convergence of their modeling error as well as a priori error estimates in the $W^{1, p}$ norm for FE-HMM applied to periodic problems with $p$-structure for $p=2$ have been derived. In contrast, our results are valid for general monotone maps $\mathcal{A}^{\varepsilon}$ satisfying $\left(\mathcal{A}_{0-2}\right)$ without assuming that $\mathcal{A}^{\varepsilon}$ has an associated scalar potential $^{2}$.

\footnotetext{
${ }^{2}$ For example, maps $\mathcal{A}^{\varepsilon}(x, \xi)=a^{\varepsilon}(x) \xi$ with a non-symmetric tensor $a^{\varepsilon}$ positive definite and bounded (linear problem) or $\mathcal{A}^{\varepsilon}(x, \xi)=a^{\varepsilon}(x)\left(1+\left(1+\sum_{i=1}^{d} \xi_{i}^{4}\right)^{-1 / 4}\right) \xi$ (nonlinear problems) with $a^{\varepsilon}$ positive definite and bounded are allowed in our analysis but not in [29].
} 
In this article, we introduce a multiscale method to solve the nonlinear monotone parabolic multiscale problem (1.1) following the design principles of the finite element heterogeneous multiscale method (FE-HMM), see $[4,23]$. Based on a homogenization result ensuring the existence of an effective model associated to (1.1), we solve the effective problem using a macro finite element method and the implicit Euler scheme for time integration. While the effective problem is (in general) not available in closed form, we approximate the effective properties of the map $\mathcal{A}^{\varepsilon}$ by upscaling the available micro information. This is achieved by solving nonlinear monotone elliptic PDEs (constrained by the macro state) using a microscopic finite element method within micro domains which are of the size of the finest scale $\varepsilon$. The computational complexity of the multiscale method is thus independent of the smallest scale $\varepsilon$.

We briefly summarize the main contributions of this paper.

First, for general $p$, we prove that the numerical solution converges in the $L^{p}\left(W^{1, p}\right)$ and $\mathcal{C}^{0}\left(L^{2}\right)$ norms towards the weak homogenized solution $u^{0}$ under a modeling assumption that can be proved in specific situations (e.g., for maps $\mathcal{A}^{\varepsilon}(x, \xi)$ locally periodic in $x$ ). To show such a convergence result, spatial errors coming from macro and micro meshes in the FE-HMM, the time discretization error and the error coming from the variational crimes (as the FE-HMM relies on numerical quadrature) need to be controlled.

Second, for strongly monotone and globally Lipschitz maps $\mathcal{A}^{\varepsilon}$ (i.e., $p=2$ and $\alpha=1, \beta=2$ in $\left.\left(\mathcal{A}_{1-2}\right)\right)^{3}$, we derive sharp error estimates in both $L^{2}\left(H^{1}\right)$ and $\mathcal{C}^{0}\left(L^{2}\right)$ norms by splitting the overall error into a modeling error, for which explicit bounds can be derived under structural assumptions on the spatial heterogeneities of $\mathcal{A}^{\varepsilon}$ (e.g., such as periodicity or random stationarity), and numerical discretization errors which we bound with respect to the time step size and the mesh size of macro as well as micro space discretizations (balancing micro and macro meshes is crucial to get a given precision at minimal computational cost). Our analysis is considerably more involved than the error analysis for single scale parabolic monotone problems [19,21]. Indeed as the homogenized data can be computed only at quadrature points of the macro mesh, we have a FEM with numerical quadrature at the macro scale and thus commit variational crimes. Further, the homogenized data are recovered from micro scale computations, hence we only obtain at quadrature points an approximation of the true homogenized map. Finally, the errors due to the sampling domains' sizes and artificial boundary conditions need also to be quantified and in contrast to $[1,3,9,24]$ our numerical strategy involves nonlinear micro problems.

The convergence results derived in this article, further serve as important ingredient for the a priori error analysis in [10], where we study a linearized variant of the proposed multiscale method for problems (1.1) with maps $\mathcal{A}^{\varepsilon}$ decomposed as $\mathcal{A}^{\varepsilon}(x, \xi)=a^{\varepsilon}(x, \xi) \xi$, where $a^{\varepsilon}(x, \xi) \in \mathbb{R}^{d \times d}$. In particular, at each timestep, we obtain linearized macro and micro equations by using the computed solution at the preceding timestep as argument $\xi$ in the tensor $a^{\varepsilon}(x, \xi)$.

Let us close the introduction by putting our results in contrast to existing FE approximation results of single scale parabolic monotone problems. In the $L^{p}\left(W^{1, p}\right)$ setting, optimal explicit convergence rates in terms of the discretization parameters have been derived for maps with a $p$-structure ${ }^{4}, e . g$. , the parabolic $p$-Laplacian, using quasi-norms in space, see $[11,20]$. Note however that under the assumptions $\left(\mathcal{A}_{1-2}\right)$ the maps $\mathcal{A}^{\varepsilon}$ have $p$-structure if and only if $\alpha=1$ and $\beta=2$. As we assume $0<\alpha \leq \min \{p-1,1\}$ and $\max \{2, p\} \leq \beta<\infty$ (the most general assumptions on the oscillatory maps allowing for homogenization, see $[15,18,42]$ ), we have in addition that $p=2$ if we want both a $p$-structure and a valid homogenization setting. For this set of parameters, the quasi-norm (in space) from $[11,20]$ collapses to the standard $H^{1}(\Omega)$ norm. For all other values of $p$, homogenization theory seems not to exist for maps $\mathcal{A}^{\varepsilon}$ with $p$-structure and thus studying numerical homogenization methods makes no sense. Therefore, convergence rates with a $p$-structure are derived for $p=2$ and $\alpha=1, \beta=2$ in $\left(\mathcal{A}_{1-2}\right)$ for our numerical homogenization method. In the case $p=2$ the error analysis for single scale parabolic monotone problems in the energy norm $\left(L^{2}\left(H^{1}\right)\right)$ is straightforward and follows the arguments of the linear case [48]. Optimal convergence rates in the $L^{2}$ norm are however more involved. So far the only results existing in the

\footnotetext{
${ }^{3}$ The case of strongly monotone and Lipschitz continuous operators in the Hilbert space setting is commonly identified as a particular case of general monotone operators in the Banach space setting (see [53], Chap. 25).

${ }^{4}|\mathcal{A}(\xi)-\mathcal{A}(\eta)| \leq L\left(\kappa_{1}+|\xi|+|\eta|\right)^{p-2}|\xi-\eta|, \quad(\mathcal{A}(\xi)-\mathcal{A}(\eta)) \cdot(\xi-\eta) \geq \lambda\left(\kappa_{2}+|\xi|+|\eta|\right)^{p-2}|\xi-\eta|^{2}, \quad \forall \xi, \eta \in \mathbb{R}^{d}$.
} 
literature for the $L^{2}$ estimates are either not optimal (see [19], where a nonlinear elliptic projection is used), restricted to the dimension $d=2$, see [50], or are a corollary of maximum norm error estimates using weighted norm techniques following $[21,28]$. In this paper we use a linear elliptic projection at the macroscopic scale to derive optimal convergence results in the $L^{2}$ norm of our FEM with numerical quadrature. For single scale parabolic problems, i.e., when no micro sampling is required, we get as a byproduct of our analysis optimal convergence rates in the $L^{2}$ norm for classical FEM (with or without numerical integration) under similar regularity assumptions as used in [19,21,50], but avoiding weighted norm techniques and valid in dimension $d \leq 3$. Further, based on this linear elliptic projection, new higher-order FE error estimates for elliptic monotone (multiscale or single scale) PDEs can be proved in [6].

The outline of this article is as follows. In Section 2, we introduce the effective model associated to the model problem (1.1). Then, we define in Section 3 a multiscale method based on a numerical upscaling procedure. In Section 4 we present our main results: the convergence of the FE-HMM in the $L^{p}\left(W^{1, p}\right)$ setting and the explicit convergence rates for strongly monotone and globally Lipschitz maps. The proofs of our main results are given in Section 5. In Section 6 we discuss an implementation of the proposed method and provide several numerical tests that illustrate our theoretical findings. In Section 7, we conclude the article with some remarks about possible generalizations and future research.

Notation 1.1. In what follows, $C$ denotes a generic positive constant, whose value can change at any occurrence. For $D \subset \mathbb{R}^{m}$, we use $\mathcal{C}^{k}\left(D, \mathbb{R}^{n}\right)$ for the set of $k$-times continuously differentiable functions $g: D \rightarrow \mathbb{R}^{n}$. For $1 \leq p \leq \infty$ (with dual exponent $p^{\prime}=p /(p-1)$ ), we consider the usual Sobolev spaces $W^{k, p}(\Omega)$. For $k=1$, $W_{0}^{1, p}(\Omega)$ is the subspace of functions with vanishing trace on the boundary $\partial \Omega$ (whose dual space is denoted by $\left(W_{0}^{1, p}(\Omega)\right)^{\prime}$ with dual pairing on $\left(W_{0}^{1, p}\right)^{\prime} \times W_{0}^{1, p}$ written as $\left.\langle\cdot, \cdot\rangle\right)$, and $\mathcal{W}_{\text {per }}^{1, p}(Y)=\left\{v \in W_{\text {per }}^{1, p}(Y) \mid \int_{Y} v(y) \mathrm{d} y=0\right\}$ where $W_{\text {per }}^{1, p}(Y)$ is defined as the closure of $\mathcal{C}_{\text {per }}^{\infty}(Y)$ (the subset of $\mathcal{C}^{\infty}\left(\mathbb{R}^{d}\right)$ of periodic functions in $Y=(0,1)^{d}$ ) for the $W^{1, p}$ norm. For $p=2$, we use the notation $H^{k}(\Omega)$ and $H_{0}^{1}(\Omega)$ (with dual space written as $H^{-1}(\Omega)$ ), respectively. For $g:[0, T] \rightarrow X$ with Banach space $\left(X,\|\cdot\|_{X}\right)$ the time derivative of $g$ is denoted by $\partial_{t} g(t)$. The space of $L^{p}$ functions $g$ and continuous functions $g$ with values in $X$ is denoted by $L^{p}(0, T ; X)$ and $\mathcal{C}^{0}([0, T], X)$, respectively. Both spaces form a Banach space when endowed with the norm $\|g\|_{L^{p}(0, T ; X)}=\left(\int_{0}^{T}\|g(t)\|_{X}^{p} \mathrm{~d} t\right)^{1 / p}$ and $\|g\|_{\mathcal{C}^{0}([0, T], X)}=\sup _{t \in[0, T]}\|g(t)\|_{X}$, respectively. The Euclidean norm for $b \in \mathbb{R}^{d}$ and the Frobenius norm for $a \in \mathbb{R}^{d \times d}$ are denoted by $|b|$ and $\|a\|_{\mathcal{F}}$, respectively, and the canonical basis of $\mathbb{R}^{d}$ is given by $e_{1}, \ldots, e_{d}$.

\section{Homogenization OF THE MODEL PROBLEM}

In this section we describe the homogenization results for the considered class of problems (1.1). Recall that we assume $p \in \mathbb{R}$ with $1<p<\infty$ such that $p>2 d /(d+2)$, i.e., $W^{1, p}(\Omega) \hookrightarrow L^{2}(\Omega)$ is a compact embedding. The problem (1.1) then has a weak formulation (in time and space) in the $W^{1, p}$ setting if $f \in L^{p^{\prime}}(\Omega), g \in L^{2}(\Omega)$ and $\mathcal{A}^{\varepsilon}$ have the property that $\mathcal{A}^{\varepsilon}(\cdot, \xi): \Omega \rightarrow \mathbb{R}^{d}$ is Lebesgue measurable for every $\xi \in \mathbb{R}^{d}$. Existence and uniqueness of a solution to problem (1.1) is studied in the Banach space

$$
E=\left\{v \in L^{p}\left(0, T ; W_{0}^{1, p}(\Omega)\right) \mid \partial_{t} v \in L^{p^{\prime}}\left(0, T ;\left(W_{0}^{1, p}(\Omega)\right)^{\prime}\right)\right\},
$$

endowed with the norm $\|v\|_{E}=\|v\|_{L^{p}\left(0, T ; W_{0}^{1, p}(\Omega)\right)}+\left\|\partial_{t} v\right\|_{L^{p^{\prime}\left(0, T ;\left(W_{0}^{1, p}(\Omega)\right)^{\prime}\right)}}$ and which satisfies the continuous embedding $E \hookrightarrow \mathcal{C}^{0}\left([0, T], L^{2}(\Omega)\right)$. Under the assumptions $\left(\mathcal{A}_{0-2}\right)$ the problem (1.1) has a unique solution $u^{\varepsilon} \in E$ for $\varepsilon>0$ (e.g., see [53], Thm. 30.A), which are uniformly bounded (with respect to $\varepsilon$ )

$$
\left\|u^{\varepsilon}\right\|_{L^{p}\left(0, T ; W_{0}^{1, p}(\Omega)\right)}^{p}+\left\|\partial_{t} u^{\varepsilon}\right\|_{L^{p^{\prime}\left(0, T ;\left(W_{0}^{1, p}(\Omega)\right)^{\prime}\right)}}^{p^{\prime}} \leq C\left(\left(L_{0}+\kappa_{1}+\kappa_{2}\right)^{p}+\|f\|_{L^{p^{\prime}}(\Omega)}^{p^{\prime}}+\|g\|_{L^{2}(\Omega)}^{2}\right),
$$

with $L_{0}$ defined in (3.15). Thus, $\left\{u^{\varepsilon}\right\}$ is a bounded sequence in $E$ and by compactness there exists a subsequence, still denoted by $\left\{u^{\varepsilon}\right\}$, and some $u^{0} \in E$, such that

$$
u^{\varepsilon} \rightarrow u^{0} \text { in } L^{p}\left(0, T ; W_{0}^{1, p}(\Omega)\right) \quad \text { and } \quad \partial_{t} u^{\varepsilon} \rightarrow \partial_{t} u^{0} \text { in } L^{p^{\prime}}\left(0, T ;\left(W_{0}^{1, p}(\Omega)\right)^{\prime}\right), \quad \text { for } \varepsilon \rightarrow 0 .
$$


The idea of homogenization is to find a limiting equation for $u^{0}$. For the problem (1.1) with $\left(\mathcal{A}_{0-2}\right)$, this question is studied in terms of $G$-convergence of parabolic operators, sometimes referred to as $P G$-convergence or strong $G$-convergence, see $[42,44]$. It can be shown that there exists a subsequence of $\left\{u^{\varepsilon}\right\}$, still denoted by $\left\{u^{\varepsilon}\right\}$, and a map $\mathcal{A}^{0}: \Omega \times \mathbb{R}^{d} \rightarrow \mathbb{R}^{d}$ (independent of $f$ ), such that $u^{\varepsilon}$ weakly converges to $u^{0}$ in the sense of $(2.2)$ and $\mathcal{A}^{\varepsilon}\left(x, \nabla u^{\varepsilon}\right) \rightarrow \mathcal{A}^{0}\left(x, \nabla u^{0}\right)$ weakly in $L^{p^{\prime}}\left(0, T ;\left(L^{p^{\prime}}(\Omega)\right)^{d}\right)$, where $u^{0} \in E$ is the solution of the homogenized or effective problem

$$
\begin{gathered}
\partial_{t} u^{0}(x, t)-\operatorname{div}\left(\mathcal{A}^{0}\left(x, \nabla u^{0}(x, t)\right)\right)=f(x) \text { in } \Omega \times(0, T), \\
u^{0}(x, t)=0 \text { on } \partial \Omega \times(0, T), \quad u^{0}(x, 0)=g(x) \text { in } \Omega,
\end{gathered}
$$

where $\mathcal{A}^{0}$ satisfies $\left(\mathcal{A}_{0-2}\right)$ with Hölder exponent $\gamma=\alpha /(\beta-\alpha)$ in $\left(\mathcal{A}_{1}\right)$ and with possibly different constants $C_{0}, \kappa_{1}, \kappa_{2}, \lambda$ and $L$. Note that the class of maps $\mathcal{A}^{\varepsilon}$ given by assumptions $\left(\mathcal{A}_{0-2}\right)$ is closed under $P G$-convergence, i.e., $\gamma=\alpha$, if and only if $p=2, \alpha=1, \beta=2$. For maps $\mathcal{A}^{\varepsilon}$ with additional structure, e.g., $\mathcal{A}^{\varepsilon}(x, \xi)=\mathcal{A}(x / \varepsilon, \xi)$ with $\mathcal{A}(y, \xi)$ a $Y$-periodic function in $y$, an explicit representation of $\mathcal{A}^{0}$ can be derived, see (5.27), and thus the whole sequence $\left\{u^{\varepsilon}\right\}$ converges to $u^{0}$ in the sense of (2.2).

We close this section by giving some examples of maps $\mathcal{A}^{\varepsilon}$ satisfying $\left(\mathcal{A}_{0-2}\right)$.

Example 1. One might consider maps $\mathcal{A}^{\varepsilon}(x, \xi)=a^{\varepsilon}(x, \xi) \xi$ where $a^{\varepsilon}(\cdot, \xi) \in\left(L^{\infty}(\Omega)\right)^{d \times d}$ (for $\left.\xi \in \mathbb{R}^{d}\right)$ is depending on $\xi$. Note that adequate conditions have to be imposed on $a^{\varepsilon}$ such that $\mathcal{A}^{\varepsilon}$ satisfies $\left(\mathcal{A}_{1-2}\right)$.

The multiscale $p$-Laplacian is a particular example. Let $b^{\varepsilon}(x) \in L^{\infty}(\Omega)$ with $0<\lambda \leq b^{\varepsilon}(x) \leq \Lambda$ a.e. $x \in \Omega$ and every $\varepsilon>0$ for some $\lambda, \Lambda \in \mathbb{R}$. Then,

$$
\mathcal{A}^{\varepsilon}(x, \xi)=b^{\varepsilon}(x)|\xi|^{p-2} \xi, \quad x \in \Omega, \xi \in \mathbb{R}^{d},
$$

satisfies $\left(\mathcal{A}_{0-2}\right)$ with $C_{0}=0, \alpha=\min \{p-1,1\}$ and $\beta=\max \{2, p\}$ (see [15], Sect. 7, [42], Sect. 2.1.3).

If $p=2$ and $\alpha=1, \beta=2$ in $\left(\mathcal{A}_{1-2}\right)$, we obtain the class of strongly monotone and globally Lipschitz continuous maps $\mathcal{A}^{\varepsilon}$, which already contains relevant problems for applications, see Examples 2 and 3.

Example 2. For linear maps $\mathcal{A}^{\varepsilon}(x, \xi)$ given by

$$
\mathcal{A}^{\varepsilon}(x, \xi)=a^{\varepsilon}(x) \xi, \quad \text { with } a^{\varepsilon}(x) \in\left(L^{\infty}(\Omega)\right)^{d \times d}, \quad \varepsilon>0,
$$

with a uniformly elliptic and bounded family of tensors $a^{\varepsilon}$ the maps $\mathcal{A}^{\varepsilon}$ satisfy $\left(\mathcal{A}_{0-2}\right)$ for $p=2, \alpha=1, \beta=2$ and with constants $C_{0}=0$ and we recover the linear parabolic multiscale problems studied in [8].

Example 3. Let $\mu^{\varepsilon}: \Omega \times \mathbb{R} \rightarrow \mathbb{R}_{\geq 0}$ be a continuous function and the maps $\mathcal{A}^{\varepsilon}$ be given by

$$
\mathcal{A}^{\varepsilon}(x, \xi)=\mu^{\varepsilon}(x,|\xi|) \xi, \quad x \in \Omega, \xi \in \mathbb{R}^{d},
$$

which is an extension of the problems studied in [35] to a multiscale context. If $\mu^{\varepsilon}(x, \cdot)$ is uniformly (in $\varepsilon$ and $x$ ) Lipschitz continuous and strongly monotone then the assumptions $\left(\mathcal{A}_{0-2}\right)$ for $p=2, \alpha=1$ and $\beta=2$ are valid for $\mathcal{A}^{\varepsilon}$, see [35]. We mention for instance Carreau laws, used to model non-Newtonian fluids, which behave for fixed $\varepsilon>0$ and $x \in \Omega$ as $\mu^{\varepsilon}(x,|\xi|) \sim 1+\left(1+|\xi|^{2}\right)^{\theta-1}$ where $1 / 2<\theta \leq 1$.

\section{Multiscale method}

In this section, we propose a multiscale method to solve nonlinear monotone parabolic multiscale problems with general spatial heterogeneities. We introduce then a reformulation of that method which is convenient for the analysis and show the existence, uniqueness and boundedness of the numerical solution. 


\subsection{FE-HMM for nonlinear monotone parabolic problems}

The multiscale method studied in this article requires a macroscopic spatial discretization of $\Omega$.

Macro discretization. Let $\mathcal{T}_{H}$ be a family of macro partitions of $\Omega$ consisting of conforming, shape-regular meshes with simplicial elements. We assume that the elements $K \in \mathcal{T}_{H}$ are open and satisfy $\cup_{K \in \mathcal{T}_{H}} \bar{K}=\bar{\Omega}$ (recall that $\Omega$ is polygonal). The macro mesh size $H$ is defined by $H=\max _{K \in \mathcal{T}_{H}} \operatorname{diam} K$, where $\operatorname{diam} K \operatorname{denotes}$ the diameter of $K \in \mathcal{T}_{H}$. Then, we consider the macro finite element space

$$
S_{0}^{1}\left(\Omega, \mathcal{T}_{H}\right)=\left\{v^{H} \in W_{0}^{1, p}(\Omega)\left|v^{H}\right|_{K} \in \mathcal{P}^{1}(K), \forall K \in \mathcal{T}_{H}\right\},
$$

where $\mathcal{P}^{1}(K)$ is the space of affine polynomials on $K \in \mathcal{T}_{H}$. Further, the multiscale method is based on barycentric quadrature

$$
\int_{\Omega} \varphi(x) \mathrm{d} x \approx \sum_{K \in \mathcal{T}_{H}}|K| \varphi\left(x_{K}\right), \quad \varphi \in \mathcal{C}^{0}(\Omega),
$$

where $x_{K}$ and $|K|$ denote the barycenter and the measure of $K \in \mathcal{T}_{H}$, respectively. We note that the quadrature formula (3.2) is exact for piecewisely affine functions $\varphi$. Further, for any macro element $K \in \mathcal{T}_{H}$ we define the sampling domain $K_{\delta}$ located at the quadrature point $x_{K}$

$$
K_{\delta}=x_{K}+\delta I, \quad \text { where } I=(-1 / 2,1 / 2)^{d} \text { and } \delta \geq \varepsilon .
$$

Within the sampling domains, micro simulations are performed to recover the upscaled data.

Multiscale method. Let the time interval $(0, T)$ be uniformly divided into $N$ subintervals of length $\Delta t=T / N$ and define $t_{n}=n \Delta t$ for $0 \leq n \leq N$ and $N \in \mathbb{N} \backslash\{0\}$. For given $u_{0}^{H} \in S_{0}^{1}\left(\Omega, \mathcal{T}_{H}\right)$, we propose the following multiscale method to capture the effective solution of (1.1): for $0 \leq n \leq N-1$, find $u_{n+1}^{H} \in S_{0}^{1}\left(\Omega, \mathcal{T}_{H}\right)$ such that

$$
\int_{\Omega} \frac{u_{n+1}^{H}-u_{n}^{H}}{\Delta t} w^{H} \mathrm{~d} x+B^{H}\left(u_{n+1}^{H} ; w^{H}\right)=\int_{\Omega} f w^{H} \mathrm{~d} x, \quad \forall w^{H} \in S_{0}^{1}\left(\Omega, \mathcal{T}_{H}\right),
$$

with the nonlinear macro map $B^{H}$ given by

$$
B^{H}\left(v^{H} ; w^{H}\right)=\sum_{K \in \mathcal{T}_{H}} \frac{|K|}{\left|K_{\delta}\right|} \int_{K_{\delta}} \mathcal{A}^{\varepsilon}\left(x, \nabla v_{K}^{h}\right) \mathrm{d} x \cdot \nabla w^{H}\left(x_{K}\right), \quad v^{H}, w^{H} \in S_{0}^{1}\left(\Omega, \mathcal{T}_{H}\right),
$$

where $v_{K}^{h}$ solve the constrained micro problems (3.6) on the sampling domains $K_{\delta}$.

Micro solver. Each sampling domain $K_{\delta}$, associated to a macro element $K \in \mathcal{T}_{H}$, is discretized by a micro mesh $\mathcal{T}_{h}$ consisting of simplicial elements $T \in \mathcal{T}_{h}$. The micro mesh size $h$ is defined by $h=\max _{T \in \mathcal{T}_{h}} \operatorname{diam} T$ and we consider the micro finite element space

$$
S^{1}\left(K_{\delta}, \mathcal{T}_{h}\right)=\left\{v^{h} \in W\left(K_{\delta}\right)\left|v^{h}\right|_{T} \in \mathcal{P}^{1}(T), \forall T \in \mathcal{T}_{h}\right\},
$$

where $\mathcal{P}^{1}(T)$ is the space of affine polynomials on $T \in \mathcal{T}_{h}$ and $W\left(K_{\delta}\right) \subset W^{1, p}\left(K_{\delta}\right)$ is some Sobolev space. The choice of the space $W\left(K_{\delta}\right)$ determines the coupling between the macro and micro solver. We consider

- periodic coupling: $W\left(K_{\delta}\right)=\mathcal{W}_{\text {per }}^{1, p}\left(K_{\delta}\right)=\left\{v \in W_{\text {per }}^{1, p}\left(K_{\delta}\right) \mid \int_{K_{\delta}} v \mathrm{~d} x=0\right\}$;

- Dirichlet coupling: $W\left(K_{\delta}\right)=W_{0}^{1, p}\left(K_{\delta}\right)$.

For $v^{H} \in S_{0}^{1}\left(\Omega, \mathcal{T}_{H}\right)$ and sampling domain $K_{\delta}$, we consider the micro problem: find $v_{K}^{h}-v^{H} \in S^{1}\left(K_{\delta}, \mathcal{T}_{h}\right)$ such that

$$
\int_{K_{\delta}} \mathcal{A}^{\varepsilon}\left(x, \nabla v_{K}^{h}\right) \cdot \nabla z^{h} \mathrm{~d} x=0, \quad \forall z^{h} \in S^{1}\left(K_{\delta}, \mathcal{T}_{h}\right),
$$

i.e., $v_{K}^{h}$ is the finite element solution to an elliptic nonlinear monotone PDE. Note that the generalization of the map $B^{H}$ and the micro problems (3.6) to higher order macro and micro FEM is given in [6]. 


\subsection{A useful reformulation of the FE-HMM}

First, in what follows, we write the difference quotient with respect to time, like in (3.3), as $\bar{\partial}_{t} v_{n}=$ $\Delta t^{-1}\left(v_{n+1}-v_{n}\right)$, for a sequence $\left\{v_{n}\right\}_{n \geq 0} \subset L^{2}(\Omega)$ and $n \geq 0$.

For the analysis of the FE-HMM it is convenient to reformulate the nonlinear map $B^{H}$ as a standard finite element method applied to a modified macro problem. Let $\xi \in \mathbb{R}^{d}$ and $K \in \mathcal{T}_{H}$, we introduce the function $\chi_{K}^{\xi, h}$ as the solution to the variational problem: find $\chi_{K}^{\xi, h} \in S^{1}\left(K_{\delta}, \mathcal{T}_{h}\right)$ such that

$$
\int_{K_{\delta}} \mathcal{A}^{\varepsilon}\left(x, \xi+\nabla \chi_{K}^{\xi, h}\right) \cdot \nabla z^{h} \mathrm{~d} x=0, \quad \forall z^{h} \in S^{1}\left(K_{\delta}, \mathcal{T}_{h}\right) .
$$

Similarly, we define $\bar{\chi}_{K}^{\xi}$ by the variational problem: find $\bar{\chi}_{K}^{\xi} \in W\left(K_{\delta}\right)$ such that

$$
\int_{K_{\delta}} \mathcal{A}^{\varepsilon}\left(x, \xi+\nabla \bar{\chi}_{K}^{\xi}\right) \cdot \nabla z \mathrm{~d} x=0, \quad \forall z \in W\left(K_{\delta}\right) .
$$

Based on the functions $\chi_{K}^{\xi, h}$ and $\bar{\chi}_{K}^{\xi}$ we define the maps

$$
\mathcal{A}_{K}^{0, h}(\xi)=\frac{1}{\left|K_{\delta}\right|} \int_{K_{\delta}} \mathcal{A}^{\varepsilon}\left(x, \xi+\nabla \chi_{K}^{\xi, h}\right) \mathrm{d} x, \quad \overline{\mathcal{A}}_{K}^{0}(\xi)=\frac{1}{\left|K_{\delta}\right|} \int_{K_{\delta}} \mathcal{A}^{\varepsilon}\left(x, \xi+\nabla \bar{\chi}_{K}^{\xi}\right) \mathrm{d} x,
$$

and the nonlinear map $B^{H}$ given in (3.4) can then be reformulated using $\mathcal{A}_{K}^{0, h}$

$$
B^{H}\left(v^{H} ; w^{H}\right)=\sum_{K \in \mathcal{T}_{H}}|K| \mathcal{A}_{K}^{0, h}\left(\nabla v^{H}\left(x_{K}\right)\right) \cdot \nabla w^{H}\left(x_{K}\right), \quad v^{H}, w^{H} \in S_{0}^{1}\left(\Omega, \mathcal{T}_{H}\right) .
$$

Thus, the modified macro form $B^{H}$ is obtained by replacing elementwisely the exact effective map $\mathcal{A}^{0}(x, \xi)$ from the homogenized equation $(2.3)$ by the approximation $\mathcal{A}_{K}^{0, h}(\xi)$.

Further, using the effective map $\mathcal{A}^{0}$ we introduce the map $B^{0}: W_{0}^{1, p}(\Omega) \times W_{0}^{1, p}(\Omega) \rightarrow \mathbb{R}$ by

$$
B^{0}(v ; w)=\int_{\Omega} \mathcal{A}^{0}(x, \nabla v(x)) \cdot \nabla w(x) \mathrm{d} x, \quad v, w \in W_{0}^{1, p}(\Omega),
$$

and, if $\mathcal{A}^{0}(\cdot, \xi)$ has a continuous representative for every $\xi \in \mathbb{R}^{d}$ (later on ensured by (4.3)), we define the nonlinear map $\hat{B}^{0}$ as its discrete counterpart

$$
\hat{B}^{0}\left(v^{H} ; w^{H}\right)=\sum_{K \in \mathcal{T}_{H}}|K| \mathcal{A}^{0}\left(x_{K}, \nabla v^{H}\left(x_{K}\right)\right) \cdot \nabla w^{H}\left(x_{K}\right), \quad v^{H}, w^{H} \in S_{0}^{1}\left(\Omega, \mathcal{T}_{H}\right) .
$$

\subsection{Existence and uniqueness of the numerical solution}

The macro scheme (3.3) and the micro problems (3.6) are variational problems of the type $G(u ; w)=F(w)$ on Banach spaces (with $G(u ; \cdot)$ and $F$ bounded linear functionals). In this section, we show local Hölder continuity of $G(\cdot ; w)$, strict monotonicity $G(v ; v-w)-G(w ; v-w)>0$ and coercivity $\lim _{v \rightarrow \infty} G(v ; v) /\|v\|=\infty$ using the hypotheses $\left(\mathcal{A}_{0-2}\right)$. The Browder-Minty theorem ([53], Thm. 26.A) then ensures existence and uniqueness of a solution to (3.3) and (3.6).

First, we introduce in Remark 3.1 and Lemma 3.2 two important inequalities.

Remark 3.1. Let $\omega \subset \mathbb{R}^{d}$ be an open and bounded domain. For $K, M \in \mathbb{N} \backslash\{0\}$, let $1<r_{k}<\infty$ satisfying $\sum_{k=1}^{K} 1 / r_{k}=1$ and $f_{k} \in L^{r_{k}}(\omega)$ as well as $x_{k}^{(m)} \in \mathbb{R}$ for $1 \leq k \leq K, 1 \leq m \leq M$. Whenever using the Hölder inequalities (either in the continuous or the discrete setting)

$$
\int_{\omega}\left|\prod_{k=1}^{K} f_{k}(x)\right| \mathrm{d} x \leq \prod_{k=1}^{K}\left\|f_{k}\right\|_{L^{r_{k}(\omega)}}, \quad \sum_{m=1}^{M}\left|\prod_{k=1}^{K} x_{k}^{(m)}\right| \leq \prod_{k=1}^{K}\left(\sum_{m=1}^{M}\left|x_{k}^{(m)}\right|^{r_{k}}\right)^{\frac{1}{r_{k}}}
$$


we simply refer to (3.12) without giving the explicit values of $r_{i}$ as the values of $r_{k}$ are always either (i) $r_{1}=p$, $r_{2}=p^{\prime}=p /(p-1)$, (ii) $r_{1}=\beta / p, r_{2}=\beta /(\beta-p)$, (iii) $r_{1}=(p-1) /(p-1-\sigma), r_{2}=(p-1) / \sigma$ if $K=2$ or (iv) $r_{1}=p /(p-1-\sigma), r_{2}=p / \sigma, r_{3}=p$ if $K=3$, with $\beta \geq p$ and $0<\sigma \leq \min \{p-1,1\}$.

The Hölder inequality yields the following fundamental technical estimate (see [18], Lem. 3.1).

Lemma 3.2. Let $\omega \subset \mathbb{R}^{d}$ be an open and bounded domain, $1<p<\infty, \beta \geq p$ and $\kappa \geq 0$. Then, for $v, w \in W^{1, p}(\omega)$ it holds

$$
\|\nabla v-\nabla w\|_{L^{p}(\omega)} \leq\left[\kappa|\omega|^{\frac{1}{p}}+\|\nabla v\|_{L^{p}(\omega)}+\|\nabla w\|_{L^{p}(\omega)}\right]^{\frac{\beta-p}{\beta}}\left(\int_{\omega}(\kappa+|\nabla v|+|\nabla w|)^{p-\beta}|\nabla v-\nabla w|^{\beta} \mathrm{d} x\right)^{\frac{1}{\beta}} .
$$

We next analyze the existence and uniqueness of a solution to the micro problem (3.6).

Lemma 3.3. Assume that $\mathcal{A}^{\varepsilon}$ satisfies $\left(\mathcal{A}_{0-2}\right)$. Let $K \in \mathcal{T}_{H}, v^{H} \in S_{0}^{1}\left(\Omega, \mathcal{T}_{H}\right)$ and $\xi \in \mathbb{R}^{d}$. For both coupling conditions, i.e., either $W\left(K_{\delta}\right)=W_{0}^{1, p}\left(K_{\delta}\right)$ or $W\left(K_{\delta}\right)=\mathcal{W}_{\text {per }}^{1, p}\left(K_{\delta}\right)$, there exists a unique solution $v_{K}^{h}-v^{H}, \chi_{K}^{\xi, h} \in$ $S^{1}\left(K_{\delta}, \mathcal{T}_{h}\right)$ and $\bar{\chi}_{K}^{\xi} \in W\left(K_{\delta}\right)$ to the micro problems (3.6), (3.7) and (3.8), respectively.

Proof. We prove the result for the micro problem (3.6). Consider the map $a_{K}^{\xi}$ given by

$$
a_{K}^{\xi}(z ; w)=\int_{K_{\delta}} \mathcal{A}^{\varepsilon}(x, \xi+\nabla z) \cdot \nabla w \mathrm{~d} x, \quad z, w \in W\left(K_{\delta}\right),
$$

which is nonlinear in $z$ and linear $w$. Then, taking $\xi=\nabla v^{H}\left(x_{K}\right)$, the micro problem (3.6) reads as

$$
\text { find } v_{K}^{h}-v^{H} \in S^{1}\left(K_{\delta}, \mathcal{T}_{h}\right) \text { such that } \quad a_{K}^{\xi}\left(v_{K}^{h}-v^{H} ; w^{h}\right)=0, \quad \forall w^{h} \in S^{1}\left(K_{\delta}, \mathcal{T}_{h}\right) .
$$

We show that the Browder-Minty theorem ([53], Thm. 26.A) can be applied to prove the existence and uniqueness of $v_{K}^{h}-v^{H} \in S^{1}\left(K_{\delta}, \mathcal{T}_{h}\right)$. The results for the problems (3.7) and (3.8) are proved analogously.

Let $v, w, z \in W\left(K_{\delta}\right)$. First, note that $\left(\mathcal{A}_{0-1}\right)$ imply that $\mathcal{A}^{\varepsilon}$ grows at rate $p-1$ with respect to $\xi$

$$
\left|\mathcal{A}^{\varepsilon}(x, \xi)\right| \leq L\left(L_{0}^{p-1}+\left(\kappa_{1}+|\xi|\right)^{p-1}\right), \quad \text { where } L_{0}=\left(C_{0} / L\right)^{\frac{1}{p-1}}, \quad \forall \xi \in \mathbb{R}^{d}, \varepsilon>0 \text {, a.e. } x \in \Omega .
$$

The growth estimate (3.15) and (3.12) yield that the linear map $a_{K}^{\xi}(v ; \cdot)$ is bounded

$$
\left|a_{K}^{\xi}(v ; w)\right| \leq 2 L\left[\left(L_{0}+\kappa_{1}+|\xi|\right)\left|K_{\delta}\right|^{\frac{1}{p}}+\|\nabla v\|_{L^{p}\left(K_{\delta}\right)}\right]^{p-1}\|\nabla w\|_{L^{p}\left(K_{\delta}\right)} .
$$

Next, using $\left(\mathcal{A}_{1}\right)$ and $(3.12)$ we get that $a_{K}^{\xi}$ is locally Hölder continuous in its first argument as

$$
\begin{aligned}
\left|a_{K}^{\xi}(v ; w)-a_{K}^{\xi}(z ; w)\right| \leq & L\left[\left(\kappa_{1}+2|\xi|\right)\left|K_{\delta}\right|^{\frac{1}{p}}+\|\nabla v\|_{L^{p}\left(K_{\delta}\right)}+\|\nabla z\|_{L^{p}\left(K_{\delta}\right)}\right]^{p-1-\alpha} \\
& \times\|\nabla v-\nabla z\|_{L^{p}\left(K_{\delta}\right)}^{\alpha}\|\nabla w\|_{L^{p}\left(K_{\delta}\right)} .
\end{aligned}
$$

Combining Lemma 3.2 with $\left(\mathcal{A}_{2}\right)$ we obtain that $a_{K}^{\xi}$ is strictly monotone as

$$
\begin{aligned}
\|\nabla v-\nabla w\|_{L^{p}\left(K_{\delta}\right)} \leq & \lambda^{-\frac{1}{\beta}}\left[\left(\kappa_{2}+2|\xi|\right)\left|K_{\delta}\right|^{\frac{1}{p}}+\|\nabla v\|_{L^{p}\left(K_{\delta}\right)}+\|\nabla w\|_{L^{p}\left(K_{\delta}\right)}\right]^{\frac{\beta-p}{\beta}} \\
& \times\left(a_{K}^{\xi}(v ; v-w)-a_{K}^{\xi}(w ; v-w)\right)^{\frac{1}{\beta}} .
\end{aligned}
$$

Further, for $v \in W\left(K_{\delta}\right)$, we first assume $\|\nabla v\|_{L^{p}\left(K_{\delta}\right)} \geq\left(\kappa_{2}+2|\xi|\right)\left|K_{\delta}\right|^{1 / p}$ and derive from (3.17) and (3.16)

$$
\begin{aligned}
a_{K}^{\xi}(v ; v) & \geq a_{K}^{\xi}(v ; v)-a_{K}^{\xi}(0 ; v)-\left|a_{K}^{\xi}(0 ; v)\right| \\
& \geq 2^{p-\beta} \lambda\|\nabla v\|_{L^{p}\left(K_{\delta}\right)}^{p}-2 L\left[\left(L_{0}+\kappa_{1}+|\xi|\right)\left|K_{\delta}\right|^{\frac{1}{p}}\right]^{p-1}\|\nabla v\|_{L^{p}\left(K_{\delta}\right)} \\
& \geq 2^{p-\beta-1} \lambda\|\nabla v\|_{L^{p}\left(K_{\delta}\right)}^{p}-C\left(L_{0}+\kappa_{1}+\kappa_{2}+|\xi|\right)^{p}\left|K_{\delta}\right|,
\end{aligned}
$$


where Young's inequality is used in the last step and $C$ only depends on $p, \beta, \lambda$ and $L$. It remains to consider $v \in W\left(K_{\delta}\right)$ with $\|\nabla v\|_{L^{p}\left(K_{\delta}\right)}<\left(\kappa_{2}+2|\xi|\right)\left|K_{\delta}\right|^{1 / p}$. In this case, the bound (3.18) (with possibly different constant $C$ ) can be directly derived from (3.16). Hence, the lower bound (3.18) for $a_{K}^{\xi}(v ; v)$ holds for any $v \in W\left(K_{\delta}\right)$ and, in particular, $a_{K}^{\xi}$ is coercive on $W\left(K_{\delta}\right)$ for both periodic and Dirichlet coupling.

For the analysis of the macro-micro coupling, the following energy equivalence is essential.

Lemma 3.4. Assume that $\mathcal{A}^{\varepsilon}$ satisfies $\left(\mathcal{A}_{0-2}\right)$. Let $K_{\delta}$ be the sampling domain associated to a macro element $K \in \mathcal{T}_{H}$ and $v_{K}^{h}$ be the solution of the micro problem (3.6) constrained by $v^{H} \in S_{0}^{1}\left(\Omega, \mathcal{T}_{H}\right)$. Then,

$$
\left\|\nabla v^{H}\right\|_{L^{p}\left(K_{\delta}\right)} \leq\left\|\nabla v_{K}^{h}\right\|_{L^{p}\left(K_{\delta}\right)} \leq C_{e}\left(\left(L_{0}+\kappa_{1}+\kappa_{2}\right)\left|K_{\delta}\right|^{\frac{1}{p}}+\left\|\nabla v^{H}\right\|_{L^{p}\left(K_{\delta}\right)}\right),
$$

where $L_{0}$ is defined in (3.15) and $C_{e} \geq 1$ only depends on $p, \beta, \lambda$ and $L$.

Proof. Due to the convexity of $|\cdot|^{p}$ on $\mathbb{R}^{d}$ it holds that $|\eta|^{p} \geq|\xi|^{p}+p|\xi|^{p-2} \xi \cdot(\eta-\xi)$ for all $\xi, \eta \in \mathbb{R}^{d}$. Applying this inequality pointwise for $\eta=\nabla v_{K}^{h}(x)$ and $\xi=\nabla v^{H}\left(x_{K}\right)$ yields

$$
\left\|\nabla v_{K}^{h}\right\|_{L^{p}\left(K_{\delta}\right)}^{p} \geq \int_{K_{\delta}}\left|\nabla v^{H}\left(x_{K}\right)\right|^{p} \mathrm{~d} x+p\left|\nabla v^{H}\left(x_{K}\right)\right|^{p-2} \nabla v^{H}\left(x_{K}\right) \cdot \int_{K_{\delta}} \nabla v_{K}^{h}-\nabla v^{H} \mathrm{~d} x=\left\|\nabla v^{H}\right\|_{L^{p}\left(K_{\delta}\right)}^{p},
$$

where in the last step we use that $\int_{K_{\delta}} \nabla v_{K}^{h}-\nabla v^{H} \mathrm{~d} x=0$ due to the Dirichlet or periodic boundary conditions of $v_{K}^{h}-v^{H} \in W\left(K_{\delta}\right)$.

The upper bound is obtained by using $\left\|\nabla v_{K}^{h}\right\|_{L^{p}\left(K_{\delta}\right)} \leq\left\|\nabla v_{K}^{h}-\nabla v^{H}\right\|_{L^{p}\left(K_{\delta}\right)}+\left\|\nabla v^{H}\right\|_{L^{p}\left(K_{\delta}\right)}$ and setting $\xi=\nabla v^{H}\left(x_{K}\right)$ in $(3.18)$

$$
2^{p-\beta-1} \lambda\left\|\nabla v_{K}^{h}-\nabla v^{H}\right\|_{L^{p}\left(K_{\delta}\right)}^{p} \leq a_{K}^{\xi}\left(v_{K}^{h}-v^{H} ; v_{K}^{h}-v^{H}\right)+C\left(L_{0}+\kappa_{1}+\kappa_{2}+\left|\nabla v^{H}\left(x_{K}\right)\right|\right)^{p}\left|K_{\delta}\right|,
$$

where $a_{K}^{\xi}\left(v_{K}^{h}-v^{H} ; v_{K}^{h}-v^{H}\right)=0$ as $v_{K}^{h}-v^{H} \in S^{1}\left(K_{\delta}, \mathcal{T}_{h}\right)$ solves the micro problem (3.14).

Using Lemma 3.4 we prove several properties of the map $B^{H}$ from (3.4).

Lemma 3.5. Assume that $\mathcal{A}^{\varepsilon}$ satisfies $\left(\mathcal{A}_{0-2}\right)$. Let $v^{H}, w^{H}, z^{H} \in S_{0}^{1}\left(\Omega, \mathcal{T}_{H}\right)$ and the nonlinear map $B^{H}$ be given by (3.4). Then $B^{H}$ satisfies the bound

$$
\left|B^{H}\left(v^{H} ; w^{H}\right)\right| \leq C_{b}\left[L_{0}+\kappa_{1}+\kappa_{2}+\left\|\nabla v^{H}\right\|_{L^{p}(\Omega)}\right]^{p-1}\left\|\nabla w^{H}\right\|_{L^{p}(\Omega)},
$$

where $C_{b}$ depends on $p, \beta, \lambda, L$ and the measure of $\Omega$. Further, $B^{H}$ is locally Hölder continuous in its first argument with exponent $\gamma=\alpha /(\beta-\alpha)$, strictly monotone and coercive, as we have

$$
\begin{aligned}
\left|B^{H}\left(v^{H} ; z^{H}\right)-B^{H}\left(w^{H} ; z^{H}\right)\right| \leq & C\left[L_{0}+\kappa_{1}+\kappa_{2}+\left\|\nabla v^{H}\right\|_{L^{p}(\Omega)}+\left\|\nabla w^{H}\right\|_{L^{p}(\Omega)}\right]^{p-1-\gamma} \\
& \times\left\|\nabla v^{H}-\nabla w^{H}\right\|_{L^{p}(\Omega)}^{\gamma}\left\|\nabla z^{H}\right\|_{L^{p}(\Omega)}, \\
\lambda_{c}^{\frac{1}{\beta}}\left\|\nabla v^{H}-\nabla w^{H}\right\|_{L^{p}(\Omega)} \leq & {\left[\left(L_{0}+\kappa_{1}+\kappa_{2}\right)|\Omega|^{\frac{1}{p}}+\left\|\nabla v^{H}\right\|_{L^{p}(\Omega)}+\left\|\nabla w^{H}\right\|_{L^{p}(\Omega)}\right]^{\frac{\beta-p}{\beta}} } \\
& \times\left(B^{H}\left(v^{H} ; v^{H}-w^{H}\right)-B^{H}\left(w^{H} ; v^{H}-w^{H}\right)\right)^{\frac{1}{\beta}}, \\
B^{H}\left(v^{H} ; v^{H}\right) \geq & \lambda_{c}\left\|\nabla v^{H}\right\|_{L^{p}(\Omega)}^{p}-C_{c}\left(L_{0}+\kappa_{1}+\kappa_{2}\right)^{p},
\end{aligned}
$$

with $\lambda_{c}>0$ depending only on $p, \beta, \lambda$ and $C_{e}$ from Lemma 3.4, where $C$ only depends on $p, \beta, \alpha, \lambda, L$ as well as the measure of $\Omega$ and $C_{c}$ depends on the same quantities like $C$ except $\alpha$. 
Remark 3.6. If $\gamma=p-1$ the constant $C$ in (3.20) has the value $L^{p} / \lambda^{p-1}$ and if $p=\beta$ the inequalities (3.21) and (3.22) hold with constant $\lambda_{c}=\lambda$ and $\lambda_{c}=\lambda / 2$, respectively.

Proof. The bound (3.19) is derived from the growth estimate (3.15), (3.12) and the upper bound of Lemma 3.4. To prove the Hölder continuity (3.20), let $v_{K}^{h}, w_{K}^{h}$ solve the micro problem (3.6) constrained by $v^{H}$ and $w^{H}$, respectively. We first observe that Lemma $3.2,\left(\mathcal{A}_{1-2}\right)$, the micro problems (3.6) and (3.12) yield

$$
\begin{aligned}
\lambda\left\|\nabla v_{K}^{h}-\nabla w_{K}^{h}\right\|_{L^{p}\left(K_{\delta}\right)}^{\beta} & \leq \mathcal{R}\left(v_{K}^{h}, w_{K}^{h}\right)^{\beta-p} \int_{K_{\delta}}\left[\mathcal{A}^{\varepsilon}\left(x, \nabla v_{K}^{h}\right)-\mathcal{A}^{\varepsilon}\left(x, \nabla w_{K}^{h}\right)\right] \cdot\left(\nabla v_{K}^{h}-\nabla w_{K}^{h}\right) \mathrm{d} x \\
& =\mathcal{R}\left(v_{K}^{h}, w_{K}^{h}\right)^{\beta-p} \int_{K_{\delta}}\left[\mathcal{A}^{\varepsilon}\left(x, \nabla v_{K}^{h}\right)-\mathcal{A}^{\varepsilon}\left(x, \nabla w_{K}^{h}\right)\right] \cdot\left(\nabla v^{H}-\nabla w^{H}\right) \mathrm{d} x \\
& \leq L \mathcal{R}\left(v_{K}^{h}, w_{K}^{h}\right)^{\beta-\alpha-1}\left\|\nabla v_{K}^{h}-\nabla w_{K}^{h}\right\|_{L^{p}\left(K_{\delta}\right)}^{\alpha}\left\|\nabla v^{H}-\nabla w^{H}\right\|_{L^{p}\left(K_{\delta}\right)},
\end{aligned}
$$

where $\mathcal{R}\left(v_{K}^{h}, w_{K}^{h}\right)=\left(\kappa_{1}+\kappa_{2}\right)\left|K_{\delta}\right|^{1 / p}+\left\|\nabla v_{K}^{h}\right\|_{L^{p}\left(K_{\delta}\right)}+\left\|\nabla w_{K}^{h}\right\|_{L^{p}\left(K_{\delta}\right)}$ can be bounded applying Lemma 3.4

$$
\mathcal{R}\left(v_{K}^{h}, w_{K}^{h}\right) \leq 3 C_{e}\left(L_{0}+\kappa_{1}+\kappa_{2}+\left|\nabla v^{H}\left(x_{K}\right)\right|+\left|\nabla w^{H}\left(x_{K}\right)\right|\right)\left|K_{\delta}\right|^{\frac{1}{p}},
$$

where $C_{e}$ is from Lemma 3.4. Hence, using inequalities (3.24) and (3.25) we get

$$
\begin{aligned}
\left\|\nabla v_{K}^{h}-\nabla w_{K}^{h}\right\|_{L^{p}\left(K_{\delta}\right)} \leq & C\left[L_{0}+\kappa_{1}+\kappa_{2}+\left|\nabla v^{H}\left(x_{K}\right)\right|+\left|\nabla w^{H}\left(x_{K}\right)\right|\right]^{\frac{\beta-\alpha-1}{\beta-\alpha}} \\
& \times\left|\nabla v^{H}\left(x_{K}\right)-\nabla w^{H}\left(x_{K}\right)\right|^{\frac{1}{\beta-\alpha}}\left|K_{\delta}\right|^{\frac{1}{p}}
\end{aligned}
$$

where $C$ only depends on $p, \beta, \alpha, \lambda, L$. Combining estimate (3.26) with $\left(\mathcal{A}_{1}\right)$ and $(3.12)$ then proves $(3.20)$.

Next, using the lower bound of Lemma 3.4, inequality (3.23), Lemma 3.2, $\left(\mathcal{A}_{2}\right)$ and $(3.12)$ leads to

$$
\begin{aligned}
\left\|\nabla v^{H}-\nabla w^{H}\right\|_{L^{p}(\Omega)}^{p} \leq & \sum_{K \in \mathcal{T}_{H}} \frac{|K|}{\left|K_{\delta}\right|}\left\|\nabla v_{K}^{h}-\nabla w_{K}^{h}\right\|_{L^{p}\left(K_{\delta}\right)}^{p} \leq \lambda^{-\frac{p}{\beta}} \sum_{K \in \mathcal{T}_{H}} \frac{|K|}{\left|K_{\delta}\right|} \mathcal{R}\left(v_{K}^{h}, w_{K}^{h}\right)^{\frac{(\beta-p) p}{\beta}} \\
& \times\left(\int_{K_{\delta}}\left[\mathcal{A}^{\varepsilon}\left(x, \nabla v_{K}^{h}\right)-\mathcal{A}^{\varepsilon}\left(x, \nabla w_{K}^{h}\right)\right] \cdot\left(\nabla v^{H}\left(x_{K}\right)-\nabla w^{H}\left(x_{K}\right)\right) \mathrm{d} x\right)^{\frac{p}{\beta}} \\
\leq & \lambda^{-\frac{p}{\beta}}\left(\sum_{K \in \mathcal{T}_{H}} \frac{|K|}{\left|K_{\delta}\right|} \mathcal{R}\left(v_{K}^{h}, w_{K}^{h}\right)^{p}\right)^{\frac{\beta-p}{\beta}}\left(B^{H}\left(v^{H} ; v^{H}-w^{H}\right)-B^{H}\left(w^{H} ; v^{H}-w^{H}\right)\right)^{\frac{p}{\beta}},
\end{aligned}
$$

which combined with (3.25) proves (3.21). The estimate (3.21) at hand, the coercivity bound (3.22) is proved analogously to the coercivity bound (3.18) shown in Lemma 3.3.

The existence and uniqueness of the numerical solution obtained by the multiscale method (3.3) follows from the Browder-Minty theorem.

Lemma 3.7. Assume that $\mathcal{A}^{\varepsilon}$ satisfies $\left(\mathcal{A}_{0-2}\right)$. Let $z^{H} \in S_{0}^{1}\left(\Omega, \mathcal{T}_{H}\right), \Delta t>0$ as well as $f \in L^{p^{\prime}}(\Omega)$ be given and let $B^{H}$ be defined in (3.4). Then, there exists a unique $u^{H} \in S_{0}^{1}\left(\Omega, \mathcal{T}_{H}\right)$ such that

$$
\int_{\Omega} \frac{u^{H}-z^{H}}{\Delta t} w^{H} \mathrm{~d} x+B^{H}\left(u^{H} ; w^{H}\right)=\int_{\Omega} f w^{H} \mathrm{~d} x, \quad \forall w^{H} \in S_{0}^{1}\left(\Omega, \mathcal{T}_{H}\right) .
$$

Proof. For fixed $z^{H} \in S_{0}^{1}\left(\Omega, \mathcal{T}_{H}\right)$, consider the bilinear form $B^{\Delta t}$ and the linear map $l_{z^{H}}^{\Delta t}$ given by

$$
B^{\Delta t}\left(v^{H}, w^{H}\right)=\frac{1}{\Delta t} \int_{\Omega} v^{H} w^{H} \mathrm{~d} x, \quad l_{z^{H}}^{\Delta t}\left(w^{H}\right)=\int_{\Omega}\left(f+\frac{1}{\Delta t} z^{H}\right) w^{H} \mathrm{~d} x,
$$


for $v^{H}, w^{H} \in S_{0}^{1}\left(\Omega, \mathcal{T}_{H}\right)$. Then, the problem (3.27) can be written as

$$
\text { find } u^{H} \in S_{0}^{1}\left(\Omega, \mathcal{T}_{H}\right) \text { such that } \quad B^{\Delta t}\left(u^{H}, w^{H}\right)+B^{H}\left(u^{H} ; w^{H}\right)=l_{z^{H}}^{\Delta t}\left(w^{H}\right), \quad \forall w^{H} \in S_{0}^{1}\left(\Omega, \mathcal{T}_{H}\right) .
$$

Observe that $B^{\Delta t}\left(v^{H}, v^{H}\right) \geq 0$ for any $v^{H} \in S_{0}^{1}\left(\Omega, \mathcal{T}_{H}\right)$ and that the Poincaré inequality yields

$$
\left|B^{\Delta t}\left(v^{H}, w^{H}\right)\right| \leq C \Delta t^{-1}\left\|\nabla v^{H}\right\|_{L^{p}(\Omega)}\left\|\nabla w^{H}\right\|_{L^{p}(\Omega)}, \quad \forall v^{H}, w^{H} \in S_{0}^{1}\left(\Omega, \mathcal{T}_{H}\right) .
$$

Combining that with Lemma 3.5 shows that $B^{\Delta t}+B^{H}$ is linear and bounded in its second argument, hemicontinuous (see [52], Def. 26.1) in its first argument, strictly monotone and coercive. As the linear map $l_{z^{H}}^{\Delta t}(\cdot)$ is continuous, the Browder-Minty theorem ([53], Thm. 26.A) thus concludes the proof.

Finally, the boundedness of the numerical approximations (3.3) is proved.

Theorem 3.8. Assume that $\left(\mathcal{A}_{0-2}\right)$ hold and that $f \in L^{p^{\prime}}(\Omega), u_{0}^{H} \in S_{0}^{1}\left(\Omega, \mathcal{T}_{H}\right)$ are given. Then, for periodic or Dirichlet coupling and any parameter $\Delta t, H, h, \delta>0$, there exists a unique numerical solution defined by the multiscale method (3.3). Further, the numerical solution $\left\{u_{n}^{H}\right\}_{n=1}^{N}$ satisfies the bound

$$
\max _{1 \leq n \leq N}\left\|u_{n}^{H}\right\|_{L^{2}(\Omega)}^{2}+\sum_{n=1}^{N} \Delta t\left\|\nabla u_{n}^{H}\right\|_{L^{p}(\Omega)}^{p} \leq C\left(\left(L_{0}+\kappa_{1}+\kappa_{2}\right)^{p}+\|f\|_{L^{p^{\prime}(\Omega)}}^{p^{\prime}}+\left\|u_{0}^{H}\right\|_{L^{2}(\Omega)}^{2}\right),
$$

where $C$ only depends on $p, \beta, \lambda, L, T$, the measure of $\Omega$ and the Poincaré constant $C_{P}$ on $\Omega$.

Proof. The existence and uniqueness of the numerical solution defined by (3.3) follows from Lemma 3.7. To derive the a priori bound we set $w^{H}=u_{n+1}^{H}$ in (3.3), use the bound (3.22) and (3.12) to obtain

$$
\begin{aligned}
\int_{\Omega} \bar{\partial}_{t} u_{n}^{H} u_{n+1}^{H} \mathrm{~d} x+\lambda_{c}\left\|\nabla u_{n+1}^{H}\right\|_{L^{p}(\Omega)}^{p} & \leq \int_{\Omega} f u_{n+1}^{H} \mathrm{~d} x+C_{c}\left(L_{0}+\kappa_{1}+\kappa_{2}\right)^{p} \\
& \leq C_{P}\|f\|_{L^{p^{\prime}}(\Omega)}\left\|\nabla u_{n+1}^{H}\right\|_{L^{p}(\Omega)}+C_{c}\left(L_{0}+\kappa_{1}+\kappa_{2}\right)^{p},
\end{aligned}
$$

where $C_{P}$ is the Poincaré constant on $\Omega$ and $\lambda_{c}$ as well as $C_{c}$ are the constants from Lemma 3.5. As

$$
\frac{1}{2} \bar{\partial}_{t}\left\|u_{n}^{H}\right\|_{L^{2}(\Omega)}^{2} \leq \int_{\Omega} \bar{\partial}_{t} u_{n}^{H} u_{n+1}^{H} \mathrm{~d} x, \quad \text { for } 0 \leq n \leq N-1,
$$

multiplying (3.28) by $2 \Delta t$ and using Young's inequality, we get that for any $0 \leq n \leq N-1$ it holds

$$
\left\|u_{n+1}^{H}\right\|_{L^{2}(\Omega)}^{2}-\left\|u_{n}^{H}\right\|_{L^{2}(\Omega)}^{2}+\lambda_{c} \Delta t\left\|\nabla u_{n+1}^{H}\right\|_{L^{p}(\Omega)}^{p} \leq C \Delta t\left(\|f\|_{L^{p^{\prime}(\Omega)}}^{p^{\prime}}+\left(L_{0}+\kappa_{1}+\kappa_{2}\right)^{p}\right),
$$

where $C$ only depends on $p$, the Poincaré constant $C_{P}$ as well as $\lambda_{c}$ and $C_{c}$ from Lemma 3.5. Summing the last inequality from $n=0$ to $n=N-1$ concludes the proof.

\section{MAin RESUlts}

In this section we present the main results about the convergence of the numerical solution $u_{n}^{H}$ defined by the multiscale strategy (3.3) towards the exact homogenized solution $u^{0}(x, t)$. In Theorem 4.2, the convergence of the numerical solution towards the homogenized solution is proved for general $p$ and in Section 4.2 fully discrete a priori error estimates are derived for strongly monotone and Lipschitz continuous maps $\mathcal{A}^{\varepsilon}$, i.e., $p=2$ with $\alpha=1, \beta=2$ in $\left(\mathcal{A}_{1-2}\right)$.

Remark 4.1. In the model problem (1.1) we consider homogeneous Dirichlet boundary conditions and source terms $f$ independent of time. We emphasize that our results can be generalized straightforwardly for a right-hand side of the form $f(x, t)$. They also remain valid for other type of boundary conditions. 
Notation for HMM error. For the analysis, we denote by $r_{H M M}$ the overall upscaling error

$$
r_{H M M}\left(\nabla v^{H}\right)=\left(\sum_{K \in \mathcal{T}_{H}}|K|\left|\mathcal{A}^{0}\left(x_{K}, \nabla v^{H}\left(x_{K}\right)\right)-\mathcal{A}_{K}^{0, h}\left(\nabla v^{H}\left(x_{K}\right)\right)\right|^{p^{\prime}}\right)^{\frac{1}{p^{\prime}}},
$$

which, if necessary, is split into the contributions of micro and modeling error $r_{\text {mic }}$ and $r_{\text {mod }}$ defined as

$$
\begin{aligned}
r_{\text {mic }}\left(\nabla v^{H}\right) & =\left(\sum_{K \in \mathcal{T}_{H}}|K|\left|\overline{\mathcal{A}}_{K}^{0}\left(\nabla v^{H}\left(x_{K}\right)\right)-\mathcal{A}_{K}^{0, h}\left(\nabla v^{H}\left(x_{K}\right)\right)\right|^{p^{\prime}}\right)^{\frac{1}{p}}, \\
r_{\text {mod }}\left(\nabla v^{H}\right) & =\left(\sum_{K \in \mathcal{T}_{H}}|K|\left|\mathcal{A}^{0}\left(x_{K}, \nabla v^{H}\left(x_{K}\right)\right)-\overline{\mathcal{A}}_{K}^{0}\left(\nabla v^{H}\left(x_{K}\right)\right)\right|^{p^{\prime}}\right)^{\frac{1}{p^{\prime}}},
\end{aligned}
$$

where $v^{H} \in S_{0}^{1}\left(\Omega, \mathcal{T}_{H}\right), \mathcal{A}^{0}$ is the exact homogenized map from (2.3) and $\overline{\mathcal{A}}_{K}^{0}$ and $\mathcal{A}_{K}^{0, h}$ are given in (3.9). Note that the Minkowski inequality yields $r_{H M M}\left(\nabla v^{H}\right) \leq r_{\text {mic }}\left(\nabla v^{H}\right)+r_{\bmod }\left(\nabla v^{H}\right)$ for $v^{H} \in S_{0}^{1}\left(\Omega, \mathcal{T}_{H}\right)$.

\subsection{Convergence of the multiscale method}

For general $1<p<\infty$ (with the usual restriction $p>2 d /(d+2)$ ), we show that the numerical solution obtained by the HMM scheme (3.3) converges to the solution of the homogenized problem (2.3) if the numerical discretization parameters (micro and macro mesh size $h$ and $H$, respectively, and time step size $\Delta t$ ) tend to zero and upscaling parameters (coupling conditions and sampling domain size $\delta$ ) exist such that the modeling error $r_{\text {mod }}$ from (4.2b) is arbitrarily small. We emphasize that the order of the limits as stated in Theorem 4.2 cannot be interchanged in general.

Upscaling parameters such that $r_{\text {mod }}$ is small exist if for the given maps $\mathcal{A}^{\varepsilon}$ an explicit formula for the homogenized map $\mathcal{A}^{0}$ is available (like for periodic or randomly stationary spatial heterogeneities of $\mathcal{A}^{\varepsilon}$ ). Note for instance, that for locally periodic maps $\mathcal{A}^{\varepsilon}$, i.e., $\mathcal{A}^{\varepsilon}(x, \xi)=\mathcal{A}(x, x / \varepsilon, \xi)$ where $\mathcal{A}(x, y, \xi)$ is $Y$-periodic in $y$, we get $r_{\text {mod }}=0$ on $S_{0}^{1}\left(\Omega, \mathcal{T}_{H}\right)$ when replacing $\mathcal{A}^{\varepsilon}(x, \xi)$ by $\mathcal{A}\left(x_{K}, x / \varepsilon, \xi\right)$ in (3.4) as well as (3.6), taking periodic boundary conditions for (3.6) and setting $\delta=\varepsilon$. The multiscale method (3.3), being defined for general maps $\mathcal{A}^{\varepsilon}$, is however reasonable if $\mathcal{A}^{\varepsilon}$ exhibits scale-separation and its good performance is known for randomly stationary data. Further, even for maps $\mathcal{A}^{\varepsilon}$ without any structure in their spatial heterogeneities, the corresponding homogenized map $\mathcal{A}^{0}$ (in the sense of $P G$-convergence) can be approximated via explicit formulas when locally periodizing the maps $\mathcal{A}^{\varepsilon}$ (see [37], p. 155) (the "principle of periodic localization").

Theorem 4.2. Assume that $\mathcal{A}^{\varepsilon}$ satisfies $\left(\mathcal{A}_{0-2}\right)$. Let $u^{0} \in E$ be the solution to the homogenized problem (2.3) and $u_{n}^{H}$ the HMM solution obtained by (3.3) with initial conditions $u_{0}^{H}$ satisfying $\left\|g-u_{0}^{H}\right\|_{L^{2}(\Omega)} \rightarrow 0$ for $H \rightarrow 0$. Let $\mathcal{A}^{0}$ be Hölder continuous in space, i.e., there exists $0<\tilde{\gamma} \leq 1$ such that (with $L_{0} \geq 0$ from (3.15))

$$
\left|\mathcal{A}^{0}\left(x_{1}, \xi\right)-\mathcal{A}^{0}\left(x_{2}, \xi\right)\right| \leq C\left|x_{1}-x_{2}\right|^{\tilde{\gamma}}\left(L_{0}^{p-1}+\left(\kappa_{1}+|\xi|\right)^{p-1}\right), \quad \forall x_{1}, x_{2} \in \Omega, \forall \xi \in \mathbb{R}^{d} .
$$

If for any given $H>0$ and $R>0$, the coupling condition for the micro problems (3.6) and the sampling domain size $\delta$ can be chosen, such that $r_{\bmod }\left(\nabla v^{H}\right)$ from $(4.2 \mathrm{~b})$ is arbitrarily small (written as $r_{\bmod } \rightarrow 0$ ) for all $v^{H} \in S_{0}^{1}\left(\Omega, \mathcal{T}_{H}\right)$ with $\left\|\nabla v^{H}\right\|_{L^{\infty}(\Omega)} \leq R$, then we have the convergence

$$
\lim _{(\Delta t, H) \rightarrow 0} \lim _{r_{\bmod } \rightarrow 0} \lim _{h \rightarrow 0}\left[\max _{1 \leq n \leq N}\left\|u^{0}\left(\cdot, t_{n}\right)-u_{n}^{H}\right\|_{L^{2}(\Omega)}+\left(\sum_{n=0}^{N-1} \int_{t_{n}}^{t_{n+1}}\left\|\nabla u^{0}(\cdot, s)-\nabla u_{n+1}^{H}\right\|_{L^{p}(\Omega)}^{p} \mathrm{~d} s\right)^{\frac{1}{p}}\right]=0 .
$$

Following the lines of the proof of Theorem 4.2, explicit convergence rates with respect to the time step size $\Delta t$, the macro and micro mesh size $H$ and $h$, respectively, can be derived if sufficient regularity of the homogenized solution $u^{0}$ is assumed, see Remark 4.3, as then the approximation of the exact solutions (at macro and micro scale) can be obtained by nodal interpolation instead of density arguments. Note however that for $p \neq 2$, higher regularity of $u^{0}$ is not realistic even for smooth data, e.g., see $[11,20]$. 
Remark 4.3. For simplicity, assume that $p>d / 2$ and that the modeling error $r_{\text {mod }}$ vanishes $\left(e . g\right.$., if $\mathcal{A}^{\varepsilon}(x, \xi)$ is locally periodic in $x$ ). Assume that $\mathcal{A}^{\varepsilon}$ satisfies $\left(\mathcal{A}_{0-2}\right)$ and that the Hölder continuity (4.3) holds for the homogenized map $\mathcal{A}^{0}$. If the exact homogenized solution $u^{0}$ to $(2.3)$ has the regularity

$$
u^{0} \in \mathcal{C}^{0}\left([0, T], W^{2, p}(\Omega)\right), \quad \partial_{t} u^{0} \in \mathcal{C}^{0}\left([0, T], W^{2, \tilde{p}}(\Omega)\right), \quad \partial_{t}^{2} u^{0} \in \mathcal{C}^{0}\left([0, T], L^{2}(\Omega)\right),
$$

with $\tilde{p}=\min \{p, 2\}$ and the solutions $\bar{\chi}_{K}^{\xi}$ to the micro problems (3.6) for $\xi \in \mathbb{R}^{d}, K \in \mathcal{T}_{H}$ satisfy

$$
\left|\bar{\chi}_{K}^{\xi}\right|_{W^{2, p}\left(K_{\delta}\right)} \leq C \varepsilon^{-1}\left(L_{0}+\kappa_{1}+\kappa_{2}+|\xi|\right)\left|K_{\delta}\right|^{\frac{1}{p}}
$$

then the HMM approximation $u_{n}^{H}$ given by (3.3) converges towards the homogenized solution $u^{0}$ at rates

$$
\left(\sum_{n=0}^{N-1} \int_{t_{n}}^{t_{n+1}}\left\|\nabla u^{0}(\cdot, s)-\nabla u_{n+1}^{H}\right\|_{L^{p}(\Omega)}^{p} \mathrm{~d} s\right)^{\frac{1}{p}} \leq C\left(\Delta t+H^{\min \{\gamma, \tilde{\gamma}\}}+\left(\frac{h}{\varepsilon}\right)^{\gamma}\right)^{\frac{1}{\beta-1}}+C\left\|g-u_{0}^{H}\right\|_{L^{2}(\Omega)}^{\frac{2}{\beta}},
$$

where $C$ is independent of $\Delta t, H, \delta, \varepsilon$ and $h$.

\subsection{Explicit convergence rates for strongly monotone and Lipschitz maps}

For $p=2$ and $\alpha=1, \beta=2$ in $\left(\mathcal{A}_{1-2}\right)$, we derive optimal convergence rates in the $L^{2}\left(0, T ; H_{0}^{1}(\Omega)\right)$ and $\mathcal{C}^{0}\left([0, T], L^{2}(\Omega)\right)$ norm for the temporal and spatial macro error in Theorem 4.4 and for the upscaling error in Theorems 4.5 and 4.6. The error estimates always contain a modeling error, which we only explicitly bound for locally periodic data $\mathcal{A}^{\varepsilon}$, and numerical discretization errors due to temporal and spatial (macro and micro) errors, whose bounds are valid without any structural assumptions about the heterogeneities of $\mathcal{A}^{\varepsilon}$.

\subsubsection{Optimal estimates for temporal and spatial macro error}

We first explicitly quantify the time discretization and the macro finite element error committed in the multiscale method (3.3).

Theorem 4.4. Let $p=2$ and assume that $\mathcal{A}^{\varepsilon}$ satisfies $\left(\mathcal{A}_{0-2}\right)$ with $\alpha=1, \beta=2$. Let $u^{0}$ be the solution to the homogenized problem (2.3) and $u_{n}^{H}$ the HMM solution obtained from (3.3). Provided that for $\mu=1$

$$
\begin{gathered}
u^{0}, \partial_{t} u^{0} \in \mathcal{C}^{0}\left([0, T], H^{2}(\Omega)\right), \quad \partial_{t}^{2} u^{0} \in \mathcal{C}^{0}\left([0, T], L^{2}(\Omega)\right), \\
\mathcal{A}^{0}(\cdot, \xi) \in W^{\mu, \infty}\left(\Omega ; \mathbb{R}^{d}\right) \quad \text { with } \quad\left\|\mathcal{A}^{0}(\cdot, \xi)\right\|_{W^{\mu, \infty}\left(\Omega ; \mathbb{R}^{d}\right)} \leq C\left(L_{0}+|\xi|\right), \quad \forall \xi \in \mathbb{R}^{d},
\end{gathered}
$$

the following discrete $\mathcal{C}^{0}\left(L^{2}\right)$ and $L^{2}\left(H^{1}\right)$ error estimates hold

$$
\begin{aligned}
& \max _{1 \leq n \leq N} \| u^{0}\left(\cdot, t_{n}\right)-u_{n}^{H} \|_{L^{2}(\Omega)}+\left(\sum_{n=1}^{N} \Delta t\left\|\nabla u^{0}\left(\cdot, t_{n}\right)-\nabla u_{n}^{H}\right\|_{L^{2}(\Omega)}^{2}\right)^{1 / 2} \\
& \leq C\left[\Delta t+H+\max _{1 \leq n \leq N} r_{H M M}\left(\nabla \mathcal{I}_{H} u^{0}\left(\cdot, t_{n}\right)\right)+\left\|g-u_{0}^{H}\right\|_{L^{2}(\Omega)}\right],
\end{aligned}
$$

where $\mathcal{I}_{H} u^{0}$ denotes the nodal interpolant of $u^{0}$ and $C$ is independent of $\Delta t, H$ and $r_{H M M}$.

If additionally we assume that (4.4b) is satisfied for $\mu=2$ and

$$
\begin{gathered}
u^{0} \in \mathcal{C}^{0}\left([0, T], W^{2, \infty}(\Omega)\right), \quad \mathcal{A}^{0}(x, \cdot) \in W^{2, \infty}\left(\mathbb{R}^{d} ; \mathbb{R}^{d}\right), \quad \text { a.e. } x \in \Omega, \\
\mathscr{A}_{i j}^{0}, \partial_{t} \mathscr{A}_{i j}^{0} \in \mathcal{C}^{0}\left([0, T], W^{1, \infty}(\Omega)\right), \quad 1 \leq i, j, \leq d, \\
\text { quasi-uniformity of macro meshes } \mathcal{T}_{H} \text { and the elliptic regularity }(5.23),
\end{gathered}
$$


where $\mathscr{A}^{0}(x, t)=D_{\xi} \mathcal{A}^{0}\left(x, \nabla u^{0}(x, t)\right)$, then, there exists an $H_{0}>0$ such that for all $H<H_{0}$, we get the improved error estimate in the discrete $\mathcal{C}^{0}\left(L^{2}\right)$ norm

$$
\max _{1 \leq n \leq N}\left\|u^{0}\left(\cdot, t_{n}\right)-u_{n}^{H}\right\|_{L^{2}(\Omega)} \leq C\left[\Delta t+H^{2}+\max _{1 \leq n \leq N} r_{H M M}\left(\nabla \tilde{u}^{H, 0}\left(\cdot, t_{n}\right)\right)+\left\|g-u_{0}^{H}\right\|_{L^{2}(\Omega)}\right]
$$

where $\tilde{u}^{H, 0}$ is the elliptic projection (5.19) and $C$ is independent of $\Delta t, H$ and $r_{H M M}$.

Let us comment on the hypotheses of Theorem 4.4 in view of the results for linear parabolic single scale and multiscale problems, see $[8,43]$, respectively. Recall that the homogenized map $\mathcal{A}^{0}$ would be given by $\mathcal{A}^{0}(x, \xi)=a^{0}(x) \xi$ with $a^{0}(x) \in \mathbb{R}^{d \times d}$ if problem (1.1) is linear.

The temporal regularity in (4.4a) is required to obtain first order global convergence of the implicit Euler scheme. Assumption (4.4b) allows to estimate the error due to the quadrature formula (3.2) and reduces to $a_{i j}^{0} \in W^{\mu, \infty}(\Omega)$ for linear problems, which is likewise assumed in ([43], Thm. 2). Further, the hypotheses (4.5) are solely used to show the optimal convergence of the spatial macro error in the $L^{2}$ norm. Condition (4.5a) is used in combination with (4.5c) to obtain error estimates in the $W^{1, \infty}$ norm for the elliptic projection (5.19) (an application of the maximum norm error estimates for standard FEM, see [14]) and to estimate the Taylor remainder term for the map $\mathcal{A}^{0}(x, \xi)$ (with respect to $\xi$ ). Finally, assumptions (4.5b) are needed to obtain optimal estimates of $u^{0}-\tilde{u}^{H, 0}$ and $\partial_{t}\left(u^{0}-\tilde{u}^{H, 0}\right)$ in the $L^{2}$ norm, where $\tilde{u}^{H, 0}$ is the elliptic projection (5.19). For linear parabolic problems (with time-dependent data) where $\mathscr{A}^{0}(x, t)=a^{0}(x, t)$, assumptions $(4.5 \mathrm{~b})$ are comparable to the conditions used in $[8,43]$.

\subsubsection{Fully discrete space-time a priori error estimates}

We decompose the HMM upscaling error $r_{H M M}$ introduced in (4.1) into micro and modeling error $r_{\text {mic }}$ and $r_{\text {mod }}$ defined in (4.2), e.g., as in [3]. In particular, $r_{\text {mic }}$ accounts for the finite element error committed during micro simulations and $r_{\bmod }$ quantifies the quality of the micro sampling, i.e., the influence of the size of the sampling domains $K_{\delta}$ or the boundary conditions in micro problems (3.6).

First, let us assume that $\bar{\chi}_{K}^{\xi}$, the exact solutions to the micro problems (3.8), satisfy

$$
\bar{\chi}_{K}^{\xi} \in H^{2}\left(K_{\delta}\right) \text { and }\left|\bar{\chi}_{K}^{\xi}\right|_{H^{2}\left(K_{\delta}\right)} \leq C \varepsilon^{-1}\left(L_{0}+|\xi|\right) \sqrt{\left|K_{\delta}\right|},
$$

for $\xi \in \mathbb{R}^{d}, K \in \mathcal{T}_{H}$. We note that the solutions $\bar{\chi}_{K}^{\xi}$ are $H^{2}$ regular if the maps $\mathcal{A}^{\varepsilon}$ are smooth (see [38], Sect. 4), and that similar assumptions are used for linear multiscale problems (see [3], Rem. 4).

As seen in $[9,22]$ for non-symmetric linear problems, adjoint micro problems are necessary to derive sharp bounds for the micro error. We introduce a similar adjoint micro problem (5.25), denote its corresponding solutions by $\bar{X}_{K}^{\xi, j}$ and assume that

(H1*)

$$
\left\{\begin{array}{l}
(i) \bar{X}_{K}^{\xi, j} \in H^{2}\left(K_{\delta}\right) \quad \text { and }\left|\bar{X}_{K}^{\xi, j}\right|_{H^{2}\left(K_{\delta}\right)} \leq C \varepsilon^{-1} \sqrt{\left|K_{\delta}\right|}, \\
(i i) \bar{X}_{K}^{\xi, j} \in W^{1, \infty}\left(K_{\delta}\right) \text { and }\left|\bar{X}_{K}^{\xi, j}\right|_{W^{1, \infty}\left(K_{\delta}\right)} \leq C,
\end{array}\right.
$$

for $\xi \in \mathbb{R}^{d}, 1 \leq j \leq d$ and $K \in \mathcal{T}_{H}$. We note that the adjoint cell problem (5.25) is a linear elliptic problem. Thus, for smooth data, the first hypothesis in $\left(\mathbf{H} \mathbf{1}^{*}\right)$ is a classical $H^{2}$ regularity result (see [3], Rem. 4) and the $W^{1, \infty}$ regularity can be proved (see [38], Sect. 3).

Theorem 4.5. Let $p=2$ and assume that $\mathcal{A}^{\varepsilon}$ satisfies $\left(\mathcal{A}_{0-2}\right)$ with $\alpha=1, \beta=2$. Let $\mu \in\{1,2\}$, $u^{0}$ be the solution to the homogenized problem (2.3) and $u_{n}^{H}$ the HMM solution obtained from (3.3). Assume (H1), hypotheses (4.4) (either for $\mu=1$ or $\mu=2$ ) and, if $\mu=2$, that additionally (4.5) holds. Further, let the 
multiscale method (3.3) be initialized with $u_{0}^{H}$ such that $\left\|g-u_{0}^{H}\right\|_{L^{2}(\Omega)} \leq C H^{\mu}$. Then we have

$$
\begin{aligned}
\max _{1 \leq n \leq N}\left\|u^{0}\left(\cdot, t_{n}\right)-u_{n}^{H}\right\|_{L^{2}(\Omega)} & \leq C\left[\Delta t+H^{\mu}+\left(\frac{h}{\varepsilon}\right)^{\nu}+\max _{1 \leq n \leq N} r_{\bmod }\left(\nabla \mathcal{U}_{n}^{H}\right)\right], \\
\left(\sum_{n=1}^{N} \Delta t\left\|\nabla u^{0}\left(\cdot, t_{n}\right)-\nabla u_{n}^{H}\right\|_{L^{2}(\Omega)}^{2}\right)^{1 / 2} & \leq C\left[\Delta t+H+\left(\frac{h}{\varepsilon}\right)^{\nu}+\max _{1 \leq n \leq N} r_{\bmod }\left(\nabla \mathcal{U}_{n}^{H}\right)\right],
\end{aligned}
$$

for $\nu=1$, where $\mathcal{U}_{n}^{H}=\mathcal{I}_{H} u^{0}\left(\cdot, t_{n}\right)$ is the nodal interpolant of $u^{0}$ if $\mu=1$ or $\mathcal{U}_{n}^{H}=\tilde{u}^{H, 0}\left(\cdot, t_{n}\right)$ is the elliptic projection (5.19) and $H<H_{0}$ (with $H_{0}$ from Thm. 4.4) if $\mu=2$. The constant $C$ is independent of $\Delta t, H, h, \varepsilon, \delta$ and the modeling error $r_{\text {mod }}$.

If in addition $\left(\mathbf{H} 1^{*}\right)$ holds and $\mathcal{A}^{\varepsilon}(x, \cdot) \in W^{2, \infty}\left(\mathbb{R}^{d} ; \mathbb{R}^{d}\right)$ for a.e. $x \in \Omega$, then (4.6) holds for $\nu=2$.

As for linear homogenization problems, e.g., see $[1,9,22]$, we thus get the optimal quadratic micro convergence rate $(h / \varepsilon)^{2}$.

Finally, we present explicit estimates for the modeling error $r_{\text {mod }}$ supposing that the maps $\mathcal{A}^{\varepsilon}$ are locally periodic and Lipschitz continuous with respect to the macroscopic variable, i.e.,

the maps $\mathcal{A}^{\varepsilon}$ are locally periodic, i.e., $\mathcal{A}^{\varepsilon}(x, \xi)=\mathcal{A}(x, x / \varepsilon, \xi)$ with $\mathcal{A}(x, y, \xi)$ being $Y$-periodic in $y$ and satisfying (for $\xi \in \mathbb{R}^{d}$, a.e. $y \in Y$ )

$$
\left|\mathcal{A}\left(x_{1}, y, \xi\right)-\mathcal{A}\left(x_{2}, y, \xi\right)\right| \leq C\left|x_{1}-x_{2}\right|\left(L_{0}+|\xi|\right), \quad \forall x_{1}, x_{2} \in \Omega .
$$

Further, if the decomposition $\mathcal{A}^{\varepsilon}(x, \xi)=\mathcal{A}(x, x / \varepsilon, \xi)$ is explicitly known, it is advantageous to modify the multiscale method (3.3) by collocating the slow variable $x$ within the sampling domains $K_{\delta}$ at the quadrature node $x_{K}$, see (5.29). In particular, $\left.\mathcal{A}^{\varepsilon}(x, \xi)\right|_{K_{\delta}}$ is replaced by $\mathcal{A}\left(x_{K}, x / \varepsilon, \xi\right)$ in the map $B^{H}$ defined in (3.4) and the micro problems (3.6).

Theorem 4.6. Let $p=2$ and assume that $\mathcal{A}^{\varepsilon}$ satisfies $\left(\mathcal{A}_{0-2}\right)$ with $\alpha=1, \beta=2$. If $(\mathbf{H 2})$ holds, then, for any $v^{H} \in S_{0}^{1}\left(\Omega, \mathcal{T}_{H}\right)$, the modeling error $r_{\bmod }\left(\nabla v^{H}\right)$ defined in $(4.2 \mathrm{~b})$ is bounded by

$$
r_{\text {mod }}\left(\nabla v^{H}\right) \leq \begin{cases}0, & \text { if } W\left(K_{\delta}\right)=\mathcal{W}_{\text {per }}^{1,2}\left(K_{\delta}\right), \delta / \varepsilon \in \mathbb{N} \text { and } \\ C_{\text {mod }}^{1} \delta, & \left.\mathcal{A}^{\varepsilon}\right|_{K_{\delta}}=\mathcal{A}\left(x_{K}, x / \varepsilon, \xi\right) \text { collocated at } x_{K}, \\ C_{\text {mod }}^{2}(\delta+\sqrt{\varepsilon / \delta}), & \text { if } W\left(K_{\delta}\right)=\mathcal{W}_{\text {per }}^{1,2}\left(K_{\delta}\right), \delta / \varepsilon \in \mathbb{N}, \\ \text { if } W\left(K_{\delta}\right)=H_{0}^{1}\left(K_{\delta}\right), \delta>\varepsilon\end{cases}
$$

with $C_{\mathrm{mod}}^{1}$ and $C_{\mathrm{mod}}^{2}$ given by

$$
C_{\text {mod }}^{1}=C\left(L_{0}+\left\|\nabla v^{H}\right\|_{L^{2}(\Omega)}\right), \quad C_{\text {mod }}^{2}=C\left(C_{\text {mod }}^{1}+\max _{K \in \mathcal{T}_{H}}\left\|\chi^{\nabla v^{H}\left(x_{K}\right)}\left(x_{K}, \cdot\right)\right\|_{W^{1, \infty}(Y)}\right),
$$

where $\chi^{\xi}\left(x_{K}, \cdot\right)$, for $\xi \in \mathbb{R}^{d}, K \in \mathcal{T}_{H}$, denote the exact solutions to the homogenization cell problems (5.28) and $C$ is independent of $\Delta t, H, h, \varepsilon, \delta$ and $v^{H}$.

Thus combining periodic coupling and collocation is optimal for locally periodic maps $\mathcal{A}^{\varepsilon}$. Further, note that $\chi^{\xi}\left(x_{K}, \cdot\right) \in W^{1, \infty}(Y)$ is a common assumption to bound the modeling error for Dirichlet coupling (e.g., see [24], Thm. 1.2) for linear problems.

Refinement strategies. Theorem 4.5 reveals that simultaneous refinement of macro and micro meshes is needed for convergence of the spatial errors. For instance, if the modeling error is equal to zero (e.g., optimal coupling is used for locally periodic maps), then we have

$$
\begin{aligned}
\max _{1 \leq n \leq N}\left\|u^{0}\left(\cdot, t_{n}\right)-u_{n}^{H}\right\|_{L^{2}(\Omega)} & \leq C\left[\Delta t+H^{2}+\left(\frac{h}{\varepsilon}\right)^{2}\right], \\
\left(\sum_{n=1}^{N} \Delta t\left\|\nabla u^{0}\left(\cdot, t_{n}\right)-\nabla u_{n}^{H}\right\|_{L^{2}(\Omega)}^{2}\right)^{1 / 2} & \leq C\left[\Delta t+H+\left(\frac{h}{\varepsilon}\right)^{2}\right],
\end{aligned}
$$


To efficiently decrease the spatial errors in the $\mathcal{C}^{0}\left(L^{2}\right)$ and the $L^{2}\left(H^{1}\right)$ norm the spatial grids $\mathcal{T}_{H}$ and $\mathcal{T}_{h}$ have to be refined according to $h / \varepsilon \sim H$ and $h / \varepsilon \sim \sqrt{H}$, respectively. Those refinement strategies allow to obtain convergence at optimal computational cost.

Complexity. The numerical upscaling used in (3.3) leads to computational cost that are independent of the size of the small oscillations $\varepsilon$. For instance, let $N_{\text {mac }}$ and $N_{\text {mic }}$ denote the number of elements in each dimension for the macro and micro spatial discretization, respectively, using quasi-uniform meshes. Then, the macro and micro mesh sizes $H$ and $h$ scale as $H \sim 1 / N_{\text {mac }}$ and $h \sim \delta / N_{\text {mic }}$, respectively. As the size $\delta$ of the sampling domains $K_{\delta}$ is of order $\mathcal{O}(\varepsilon)$, we find that $h / \varepsilon \sim 1 / N_{\text {mic }}$. Thus, the convergence rates summarized in (4.7) can be expressed in terms of $N_{\mathrm{mac}}$ and $N_{\mathrm{mic}}$, i.e., they are robust with respect to $\varepsilon$, and can be obtained with $\mathcal{O}\left(N_{\text {mac }}^{d} N_{\text {mic }}^{d}\right)$ spatial degrees of freedom.

\section{Proof of the MAIN RESUlts}

In this section, we prove Theorems 4.2, 4.4, 4.5 and 4.6. We split the total error according to $\left\|u^{0}-u_{n}^{H}\right\| \leq$ $\left\|u^{0}-\mathcal{U}_{n}^{H}\right\|+\left\|\mathcal{U}_{n}^{H}-u_{n}^{H}\right\|$ where $\mathcal{U}_{n}^{H}$ is an approximation of the exact solution $u^{0}$ in $S_{0}^{1}\left(\Omega, \mathcal{T}_{H}\right)$. For the general convergence result of Theorem 4.2 we choose $\mathcal{U}_{n}^{H}$ to be close to $u^{0}$ using the density of smooth functions in the space $E$ given in (2.1). To derive explicit convergence rates in the case of $p=2$ and $\alpha=1, \beta=2$ in $\left(\mathcal{A}_{1-2}\right)$, choosing $\mathcal{U}_{n}^{H}$ as the nodal interpolant of $u^{0}$ yields optimal $L^{2}\left(H^{1}\right)$ estimates, whereas a new elliptic projection of $u^{0}$ for nonlinear monotone problems is needed for the optimal $\mathcal{C}^{0}\left(L^{2}\right)$ estimates.

\subsection{Error propagation formula}

Let $\mathcal{U} \in E$ with $\mathcal{U} \in \mathcal{C}^{0}\left([0, T], W_{0}^{1, p}(\Omega)\right)$ and $\partial_{t} \mathcal{U} \in \mathcal{C}^{0}\left([0, T], L^{2}(\Omega)\right)$. Further, let $\mathcal{U}^{H}(\cdot, t) \in S_{0}^{1}\left(\Omega, \mathcal{T}_{H}\right)$ be an approximation of $\mathcal{U}(\cdot, t)$ for $t \in[0, T]$ and define $\mathcal{U}_{n}^{H}=\mathcal{U}^{H}\left(\cdot, t_{n}\right)$ for $0 \leq n \leq N$. The fundamental tool to derive a priori error estimates using the energy method is the error propagation formula for the error $\theta_{n}^{H}=u_{n}^{H}-\mathcal{U}_{n}^{H}$, $0 \leq n \leq N$, given by

$$
\begin{aligned}
\Delta t & \int_{\Omega} \bar{\partial}_{t} \theta_{n}^{H} w^{H} \mathrm{~d} x+\Delta t\left[B^{H}\left(u_{n+1}^{H} ; w^{H}\right)-B^{H}\left(\mathcal{U}_{n+1}^{H} ; w^{H}\right)\right] \\
= & \int_{t_{n}}^{t_{n+1}} \int_{\Omega} f w^{H} \mathrm{~d} x \mathrm{~d} s-\Delta t \int_{\Omega} \bar{\partial}_{t} \mathcal{U}_{n}^{H} w^{H} \mathrm{~d} x-\Delta t B^{H}\left(\mathcal{U}_{n+1}^{H} ; w^{H}\right) \\
= & \int_{t_{n}}^{t_{n+1}}\left\langle\partial_{t} u^{0}(\cdot, s), w^{H}\right\rangle \mathrm{d} s-\Delta t \int_{\Omega} \bar{\partial}_{t} \mathcal{U}_{n}^{H} w^{H} \mathrm{~d} x+\int_{t_{n}}^{t_{n+1}} B^{0}\left(u^{0}(\cdot, s) ; w^{H}\right) \mathrm{d} s-\Delta t B^{H}\left(\mathcal{U}_{n+1}^{H} ; w^{H}\right) \\
= & \int_{t_{n}}^{t_{n+1}}\left\langle\partial_{t} u^{0}(\cdot, s)-\partial_{t} \mathcal{U}\left(\cdot, t_{n+1}\right), w^{H}\right\rangle \mathrm{d} s \\
& +\int_{t_{n}}^{t_{n+1}} B^{0}\left(u^{0}(\cdot, s) ; w^{H}\right)-B^{0}\left(\mathcal{U}\left(\cdot, t_{n+1}\right) ; w^{H}\right) \mathrm{d} s \\
& +\Delta t \int_{\Omega}\left[\partial_{t} \mathcal{U}\left(x, t_{n+1}\right)-\bar{\partial}_{t} \mathcal{U}\left(x, t_{n}\right)\right] w^{H} \mathrm{~d} x \\
& +\Delta t \int_{\Omega}\left[\bar{\partial}_{t} \mathcal{U}\left(x, t_{n}\right)-\bar{\partial}_{t} \mathcal{U}_{n}^{H}\right] w^{H} \mathrm{~d} x \\
& +\Delta t\left[B^{0}\left(\mathcal{U}\left(\cdot, t_{n+1}\right) ; w^{H}\right)-B^{0}\left(\mathcal{U}_{n+1}^{H} ; w^{H}\right)\right] \\
& +\Delta t\left[B^{0}\left(\mathcal{U}_{n+1}^{H} ; w^{H}\right)-\hat{B}^{0}\left(\mathcal{U}_{n+1}^{H} ; w^{H}\right)\right] \\
& +\Delta t\left[\hat{B}^{0}\left(\mathcal{U}_{n+1}^{H} ; w^{H}\right)-B^{H}\left(\mathcal{U}_{n+1}^{H} ; w^{H}\right)\right]
\end{aligned}
$$

where $w^{H} \in S_{0}^{1}\left(\Omega, \mathcal{T}_{H}\right)$ is arbitrary, $u^{0}$ is the exact solution to the homogenized problem (2.3) and the forms $B^{0}, \hat{B}^{0}$ and $B^{H}$ are given by (3.10), (3.11) and (3.4), respectively. 
In the error propagation formula (5.1) we already decomposed the overall error into its different components. The terms (5.1a) and (5.1b) arise from the difference between the weak and the strong formulation in time of (2.3). In particular, they vanish if we set $\mathcal{U}=u^{0}$ (with $u^{0}$ sufficiently smooth), see the proof of Theorem 4.4 for details. The error terms (5.1c) $-(5.1 \mathrm{~g})$ are due to the numerical discretization used for the multiscale method (3.3). While the term (5.1c) accounts for the error due to the time discretization scheme, the terms (5.1d) and (5.1e) consist of the finite element error at the discrete time levels $t_{n}$ and (5.1f) captures the influence of quadrature formula (3.2). The components (5.1c)-(5.1f) are independent of the multiscale nature of the method (3.3), whereas the last term $(5.1 \mathrm{~g})$ is solely due to the upscaling strategy consisting of micro simulations and averaging techniques. Thus we call term (5.1g) the HMM upscaling error.

In our subsequent analysis we first estimate the different error terms from (5.1), see Sections 5.1.1, 5.1.2 and 5.1.3 for estimates of the difference between the weak and strong formulation in time of (2.3), the temporal and macro spatial error and the HMM upscaling error, respectively. Those bounds at hand we then prove Theorem 4.2 in Section 5.2. Finally, for $p=2$ and $\alpha=1, \beta=2$ in $\left(\mathcal{A}_{1-2}\right)$, the proofs of Theorems 4.4 and 4.5 given in Section 5.3 follow from the improved bounds for the macro spatial error (using a linear elliptic projection) derived in Section 5.3.1 and the explicit estimates for the HMM error consisting of micro and modeling error shown in Section 5.3.2.

\subsubsection{Estimates for the difference between weak and strong formulation in time}

In this section, we estimate the terms (5.1a) and (5.1b).

Lemma 5.1. Let $u^{0}, \mathcal{U} \in E$ with $\mathcal{U}, \partial_{t} \mathcal{U}, \partial_{t}^{2} \mathcal{U} \in \mathcal{C}^{0}\left([0, T], L^{2}(\Omega)\right)$. Then, for $w^{H} \in S_{0}^{1}\left(\Omega, \mathcal{T}_{H}\right)$

$$
\begin{aligned}
\left|\int_{t_{n}}^{t_{n+1}}\left\langle\partial_{t} u^{0}(\cdot, s)-\partial_{t} \mathcal{U}\left(\cdot, t_{n+1}\right), w^{H}\right\rangle \mathrm{d} s\right| \leq & \int_{t_{n}}^{t_{n+1}}\left\|\partial_{t} u^{0}(\cdot, s)-\partial_{t} \mathcal{U}(\cdot, s)\right\|_{\left(W_{0}^{1, p}(\Omega)\right)^{\prime}}\left\|\nabla w^{H}\right\|_{L^{p}(\Omega)} \mathrm{d} s \\
& +C \Delta t^{2}\left\|\partial_{t}^{2} \mathcal{U}\right\|_{\mathcal{C}^{0}\left([0, T], L^{2}(\Omega)\right)}\left\|w^{H}\right\|_{L^{2}(\Omega)},
\end{aligned}
$$

for $0 \leq n \leq N-1$ and where $C$ is independent of $\Delta t$ and $H$.

Proof. Let $w^{H} \in S_{0}^{1}\left(\Omega, \mathcal{T}_{H}\right)$. We decompose the term into the two parts

$$
\int_{t_{n}}^{t_{n+1}}\left\langle\partial_{t} u^{0}(\cdot, s)-\partial_{t} \mathcal{U}(\cdot, s), w^{H}\right\rangle \mathrm{d} s-\int_{t_{n}}^{t_{n+1}} \int_{\Omega}\left[\partial_{t} \mathcal{U}\left(x, t_{n+1}\right)-\partial_{t} \mathcal{U}(x, s)\right] w^{H} \mathrm{~d} x \mathrm{~d} s,
$$

where the first part is bounded straightforwardly. Next, for the inner integral of the second term we have

$$
\int_{\Omega}\left[\partial_{t} \mathcal{U}\left(x, t_{n+1}\right)-\partial_{t} \mathcal{U}(x, s)\right] w^{H} \mathrm{~d} x=\int_{s}^{t_{n+1}} \int_{\Omega} \partial_{t}^{2} \mathcal{U}(x, \tau) w^{H} \mathrm{~d} x \mathrm{~d} \tau, \quad \forall s \in\left[t_{n}, t_{n+1}\right],
$$

as $\partial_{t} \mathcal{U}, \partial_{t}^{2} \mathcal{U} \in \mathcal{C}^{0}\left([0, T], L^{2}(\Omega)\right)$. The claimed result is then directly obtained.

The second term (5.1b) is bounded using the Hölder continuity of the homogenized map $\mathcal{A}^{0}$.

Lemma 5.2. Let $u^{0}, \mathcal{U} \in E$ with $\mathcal{U}, \partial_{t} \mathcal{U} \in \mathcal{C}^{0}\left([0, T], W^{1, p}(\Omega)\right)$. Assume that $\mathcal{A}^{0}$ satisfies $\left(\mathcal{A}_{1}\right)$ with exponent $\gamma=\alpha /(\beta-\alpha)$ and constants $\tilde{L}>0, \tilde{\kappa}_{1} \geq 0$. Then, for $w^{H} \in S_{0}^{1}\left(\Omega, \mathcal{T}_{H}\right)$ and $0 \leq n \leq N-1$

$$
\begin{aligned}
& \quad\left|\int_{t_{n}}^{t_{n+1}} B^{0}\left(u^{0}(\cdot, s) ; w^{H}\right)-B^{0}\left(\mathcal{U}\left(\cdot, t_{n+1}\right) ; w^{H}\right) \mathrm{d} s\right| \\
& \leq \tilde{L} \int_{t_{n}}^{t_{n+1}}\left(\tilde{\kappa}_{1}+\left\|\nabla u^{0}(\cdot, s)\right\|_{L^{p}(\Omega)}+\|\nabla \mathcal{U}(\cdot, s)\|_{L^{p}(\Omega)}\right)^{p-1-\gamma}\left\|\nabla u^{0}(\cdot, s)-\nabla \mathcal{U}(\cdot, s)\right\|_{L^{p}(\Omega)}^{\gamma}\left\|\nabla w^{H}\right\|_{L^{p}(\Omega)} \mathrm{d} s \\
& \quad+C \Delta t^{1+\gamma}\left(\tilde{\kappa}_{1}+\|\nabla \mathcal{U}\|_{\mathcal{C}^{0}\left([0, T], L^{p}(\Omega)\right)}+\left\|\partial_{t} \nabla \mathcal{U}\right\|_{\mathcal{C}^{0}\left([0, T], L^{p}(\Omega)\right)}\right)^{p-1}\left\|\nabla w^{H}\right\|_{L^{p}(\Omega)},
\end{aligned}
$$

where $C$ is independent $\Delta t$ and $H$. 
Proof. Let $w^{H} \in S_{0}^{1}\left(\Omega, \mathcal{T}_{H}\right)$. We then use the decomposition of the error term into

$$
\int_{t_{n}}^{t_{n+1}} B^{0}\left(u^{0}(\cdot, s) ; w^{H}\right)-B^{0}\left(\mathcal{U}(\cdot, s) ; w^{H}\right) \mathrm{d} s-\int_{t_{n}}^{t_{n+1}} B^{0}\left(\mathcal{U}\left(\cdot, t_{n+1}\right) ; w^{H}\right)-B^{0}\left(\mathcal{U}(\cdot, s) ; w^{H}\right) \mathrm{d} s,
$$

where the first integral is estimated using the Hölder continuity of $\mathcal{A}^{0}$ and (3.12). To bound the second term we first note that since $\mathcal{U}, \partial_{t} \mathcal{U} \in \mathcal{C}^{0}\left([0, T], W^{1, p}(\Omega)\right)$ it holds for $t_{n} \leq s \leq t_{n+1}$, similarly to (5.2),

$$
\left\|\nabla \mathcal{U}\left(\cdot, t_{n+1}\right)-\nabla \mathcal{U}(\cdot, s)\right\|_{L^{p}(\Omega)}=\left\|\int_{s}^{t_{n+1}} \partial_{t} \nabla \mathcal{U}(\cdot, \tau) \mathrm{d} \tau\right\|_{L^{p}(\Omega)} \leq \Delta t\left\|\partial_{t} \nabla \mathcal{U}\right\|_{\mathcal{C}^{0}\left([0, T], L^{p}(\Omega)\right)},
$$

which combined with the Hölder continuity of $\mathcal{A}^{0}$ and (3.12) yields the final bound.

\subsubsection{Temporal and macro spatial error}

In this section, we provide explicit error bounds for the terms (5.1c)-(5.1f).

Time discretization error. We start by estimating the error due to the implicit Euler method, i.e., term (5.1c).

Lemma 5.3. Let $\mathcal{U}, \partial_{t} \mathcal{U}, \partial_{t}^{2} \mathcal{U} \in \mathcal{C}^{0}\left([0, T], L^{2}(\Omega)\right)$. For $w^{H} \in S_{0}^{1}\left(\Omega, \mathcal{T}_{H}\right)$ and $0 \leq n \leq N-1$, we have

$$
\left|\int_{\Omega}\left[\partial_{t} \mathcal{U}\left(x, t_{n+1}\right)-\bar{\partial}_{t} \mathcal{U}\left(x, t_{n}\right)\right] w^{H} \mathrm{~d} x\right| \leq C \Delta t\left\|\partial_{t}^{2} \mathcal{U}\right\|_{\mathcal{C}^{0}\left([0, T], L^{2}(\Omega)\right)}\left\|w^{H}\right\|_{L^{2}(\Omega)},
$$

where $C$ is independent of $\Delta t$ and $H$.

Proof. Let $w^{H} \in S_{0}^{1}\left(\Omega, \mathcal{T}_{H}\right)$. As $\mathcal{U}, \partial_{t} \mathcal{U} \in \mathcal{C}^{0}\left([0, T], L^{2}(\Omega)\right)$ results similar to (5.2) hold if $\mathcal{U}$ and $\partial_{t} \mathcal{U}$ is substitute to $\partial_{t} \mathcal{U}$ and $\partial_{t}^{2} \mathcal{U}$, respectively, in particular, $\int_{\Omega} \bar{\partial}_{t} \mathcal{U}\left(x, t_{n}\right) w^{H} \mathrm{~d} x=\Delta t^{-1} \int_{t_{n}}^{t_{n+1}} \int_{\Omega} \partial_{t} \mathcal{U}(x, s) w^{H} \mathrm{~d} x \mathrm{~d} s$. Combining that with the result from (5.2) yields

$$
\int_{\Omega}\left[\partial_{t} \mathcal{U}\left(x, t_{n+1}\right)-\bar{\partial}_{t} \mathcal{U}\left(x, t_{n}\right)\right] w^{H} \mathrm{~d} x=\frac{1}{\Delta t} \int_{t_{n}}^{t_{n+1}} \int_{s}^{t_{n+1}} \int_{\Omega} \partial_{t}^{2} \mathcal{U}(x, \tau) w^{H} \mathrm{~d} x \mathrm{~d} \tau \mathrm{d} s,
$$

from where the result of Lemma 5.3 follows.

Macro finite element error. Next, we estimate the spatial macro error terms (5.1d) and (5.1e) in Lemmas 5.5 and 5.7, respectively. We therefore first introduce the nodal interpolant.

Nodal interpolant. Let $\mathcal{I}_{H}: \mathcal{C}^{0}(\bar{\Omega}) \rightarrow S^{1}\left(\Omega, \mathcal{T}_{H}\right)$ be the usual nodal interpolant where $S^{1}\left(\Omega, \mathcal{T}_{H}\right)$ is the FEspace defined as $S_{0}^{1}\left(\Omega, \mathcal{T}_{H}\right)$ in (3.1), but without zero boundary conditions. Then, for $k \in\{1,2\}$ and $q, q^{*}$ with $1 \leq q \leq q^{*}$ and $q^{*}>d / 2$, we have the bounds (see [16], Thm. 3.1.6),

$$
\begin{gathered}
\left\|\mathcal{I}_{H} z\right\|_{W^{1, q}(\Omega)} \leq C\|z\|_{W^{2, q^{*}}(\Omega)}, \quad\left\|\mathcal{I}_{H} z-z\right\|_{W^{2-k, q}(\Omega)} \leq C H^{k}\|z\|_{W^{2, q^{*}}(\Omega)}, \quad \forall z \in W^{2, q^{*}}(\Omega), \\
\left\|\mathcal{I}_{H} z\right\|_{W^{1, \infty}(\Omega)} \leq C\|z\|_{W^{1, \infty}(\Omega)}, \quad \forall z \in W^{1, \infty}(\Omega) .
\end{gathered}
$$

We note that for $z \in \mathcal{C}^{0}(\bar{\Omega}) \cap W_{0}^{1, p}(\Omega)$ it holds that $\mathcal{I}_{H} z \in S_{0}^{1}\left(\Omega, \mathcal{T}_{H}\right)$.

Remark 5.4. Let $q, q^{*}$ be as in (5.4a). If $\mathcal{U}, \partial_{t} \mathcal{U} \in \mathcal{C}^{0}\left([0, T], W^{2, q^{*}}(\Omega)\right)$, then the interpolation operator $\mathcal{I}_{H}$ and the differentiation $\partial_{t}$ with respect to the time variable can be interchanged, i.e., $\partial_{t}\left(\mathcal{I}_{H} \mathcal{U}(x, t)\right)=\mathcal{I}_{H}\left(\partial_{t} \mathcal{U}(x, t)\right)$ on $\Omega \times[0, T]$ and thus $\left\|\partial_{t} \mathcal{U}(\cdot, t)-\partial_{t}\left(\mathcal{I}_{H} \mathcal{U}(\cdot, t)\right)\right\|_{W^{1, q}(\Omega)} \leq C H\left\|\partial_{t} \mathcal{U}(\cdot, t)\right\|_{W^{2, q^{*}}(\Omega)}$.

Lemma 5.5. Let either $\tilde{p}=2$ (if $p \geq 2$ ) or $p \leq \tilde{p} \leq 2$ with $\tilde{p}>d / 2($ if $p<2)$. Let $\mathcal{U}, \partial_{t} \mathcal{U} \in \mathcal{C}^{0}\left([0, T], W^{2, \tilde{p}}(\Omega)\right.$ ) and $\mathcal{U}_{n}^{H}=\mathcal{I}_{H} \mathcal{U}\left(\cdot, t_{n}\right)$ be its nodal interpolant for $0 \leq n \leq N-1$. Then, it holds

$$
\left|\int_{\Omega}\left[\bar{\partial}_{t} \mathcal{U}\left(x, t_{n}\right)-\bar{\partial}_{t} \mathcal{U}_{n}^{H}\right] w^{H} \mathrm{~d} x\right| \leq C H\left\|\partial_{t} \mathcal{U}\right\|_{\mathcal{C}^{0}\left([0, T], W^{2, \tilde{p}}(\Omega)\right)}\left\|w^{H}\right\|_{L^{2}(\Omega)}
$$

for any $w^{H} \in S_{0}^{1}\left(\Omega, \mathcal{T}_{H}\right)$ and with a constant $C$ independent of $\Delta t$ and $H$. 
Proof. As $\mathcal{U}, \partial_{t} \mathcal{U} \in \mathcal{C}^{0}\left([0, T], W^{2, \tilde{p}}(\Omega)\right)$ and $\mathcal{I}_{H} \mathcal{U}, \partial_{t} \mathcal{I}_{H} \mathcal{U} \in \mathcal{C}^{0}\left([0, T], S_{0}^{1}\left(\Omega, \mathcal{I}_{H}\right)\right)$, see Remark 5.4, equation (5.2) holds analogously if $\partial_{t} \mathcal{U}$ is substituted by $\mathcal{U}$ or $\mathcal{U}^{H}$. Thus, for $w^{H} \in S_{0}^{1}\left(\Omega, \mathcal{T}_{H}\right)$, we obtain

$$
\begin{aligned}
\left|\int_{\Omega}\left[\bar{\partial}_{t} \mathcal{U}\left(x, t_{n}\right)-\bar{\partial}_{t} \mathcal{U}_{n}^{H}\right] w^{H} \mathrm{~d} x\right| & \leq \frac{1}{\Delta t} \int_{t_{n}}^{t_{n+1}}\left\|\partial_{t} \mathcal{U}(x, s)-\partial_{t} \mathcal{U}^{H}(x, s)\right\|_{L^{2}(\Omega)}\left\|w^{H}\right\|_{L^{2}(\Omega)} \mathrm{d} s \\
& \leq C \frac{1}{\Delta t} \int_{t_{n}}^{t_{n+1}}\left\|\partial_{t} \mathcal{U}(x, s)-\partial_{t} \mathcal{U}^{H}(x, s)\right\|_{W^{1, \tilde{p}}(\Omega)}\left\|w^{H}\right\|_{L^{2}(\Omega)} \mathrm{d} s,
\end{aligned}
$$

and the estimate from Remark 5.4 concludes the proof.

Remark 5.6. If $\tilde{p}=2$ a convergence of order $\mathcal{O}\left(H^{2}\right)$ can be shown in Lemma 5.5 by applying the interpolation estimate (5.4a) directly to (5.5), i.e., without first using the embedding $W^{1, \tilde{p}}(\Omega) \hookrightarrow L^{2}(\Omega)$.

Lemma 5.7. Assume that $\mathcal{A}^{0}$ satisfies $\left(\mathcal{A}_{1}\right)$ with $\gamma=\alpha /(\beta-\alpha)$ and constants $\tilde{L}>0, \tilde{\kappa}_{1} \geq 0$. Let $\mathcal{U} \in$ $\mathcal{C}^{0}\left([0, T], W^{2, p^{*}}(\Omega)\right)$ for some $p^{*}$ with $p \leq p^{*}$ and $p^{*}>d / 2$ and let $\mathcal{U}_{n}^{H}=\mathcal{I}_{H} \mathcal{U}\left(\cdot, t_{n}\right)$ be its nodal interpolant. Then, for $0 \leq n \leq N-1$

$$
\left|B^{0}\left(\mathcal{U}\left(\cdot, t_{n+1}\right) ; w^{H}\right)-B^{0}\left(\mathcal{U}_{n+1}^{H} ; w^{H}\right)\right| \leq C H^{\gamma}\left(\tilde{\kappa}_{1}+\|\mathcal{U}\|_{\mathcal{C}^{0}\left([0, T], W^{2, p^{*}}(\Omega)\right)}\right)^{p-1}\left\|\nabla w^{H}\right\|_{L^{p}(\Omega)},
$$

for any $w^{H} \in S_{0}^{1}\left(\Omega, \mathcal{T}_{H}\right)$ and with $C$ independent of $\Delta t$ and $H$.

Proof. Let $w^{H} \in S_{0}^{1}\left(\Omega, \mathcal{T}_{H}\right)$. The Hölder continuity $\left(\mathcal{A}_{1}\right)$ of $\mathcal{A}^{0}$ and (3.12) yield

$$
\begin{aligned}
\left|B^{0}\left(\mathcal{U}\left(\cdot, t_{n+1}\right) ; w^{H}\right)-B^{0}\left(\mathcal{U}_{n+1}^{H} ; w^{H}\right)\right| \leq & \tilde{L}\left(\tilde{\kappa}_{1}+\left\|\nabla \mathcal{U}\left(\cdot, t_{n+1}\right)\right\|_{L^{p}(\Omega)}+\left\|\nabla \mathcal{U}_{n+1}^{H}\right\|_{L^{p}(\Omega)}\right)^{p-1-\gamma} \\
& \times\left\|\nabla \mathcal{U}\left(\cdot, t_{n+1}\right)-\nabla \mathcal{U}_{n+1}^{H}\right\|_{L^{p}(\Omega)}^{\gamma}\left\|\nabla w^{H}\right\|_{L^{p}(\Omega)},
\end{aligned}
$$

which is estimated using the bounds (5.4a).

Quadrature error for Hölder continuous $\mathcal{A}^{0}$. Estimating the effect of the barycentric quadrature (3.2) used in the method (3.3) is achieved by comparing the maps $B^{0}$ and $\hat{B}^{0}$ given by (3.10) and (3.11), respectively.

Lemma 5.8. Assume that $\mathcal{A}^{0}$ satisfies the hypothesis (4.3) for some $0<\tilde{\gamma} \leq 1$. Let $B^{0}$ and $\hat{B}^{0}$ be given by (3.10) and (3.11), respectively. Then, the error due to the quadrature (3.2) is bounded by

$$
\left|B^{0}\left(v^{H} ; w^{H}\right)-\hat{B}^{0}\left(v^{H} ; w^{H}\right)\right| \leq C H^{\tilde{\gamma}}\left(L_{0}+\left\|\nabla v^{H}\right\|_{L^{p}(\Omega)}\right)^{p-1}\left\|\nabla w^{H}\right\|_{L^{p}(\Omega)},
$$

for any $v^{H}, w^{H} \in S_{0}^{1}\left(\Omega, \mathcal{T}_{H}\right)$ and where $C$ is independent of $H$.

In particular, if $\mathcal{U} \in \mathcal{C}^{0}\left([0, T], W^{2, p^{*}}(\Omega)\right)$ for some $p^{*}$ such that $p^{*}>d / 2$ and $\mathcal{U}_{n}^{H}=\mathcal{I}_{H} \mathcal{U}\left(\cdot, t_{n}\right)$ where $\mathcal{I}_{H}$ denotes the nodal interpolant, then we have for $0 \leq n \leq N-1$

$$
\left|B^{0}\left(\mathcal{U}_{n+1}^{H} ; w^{H}\right)-\hat{B}^{0}\left(\mathcal{U}_{n+1}^{H} ; w^{H}\right)\right| \leq C H^{\tilde{\gamma}}\left(L_{0}+\|\mathcal{U}\|_{\mathcal{C}^{0}\left([0, T], W^{2, p^{*}}(\Omega)\right)}\right)^{p-1}\left\|\nabla w^{H}\right\|_{L^{p}(\Omega)},
$$

for any $w^{H} \in S_{0}^{1}\left(\Omega, \mathcal{T}_{H}\right)$ and where $C$ is independent of $H$.

Proof. Let $v^{H}, w^{H} \in S_{0}^{1}\left(\Omega, \mathcal{T}_{H}\right)$. As $\nabla v^{H}$ and $\nabla w^{H}$ are piecewise constant, we get from (3.10) and (3.11)

$$
B^{0}\left(v^{H} ; w^{H}\right)-\hat{B}^{0}\left(v^{H} ; w^{H}\right)=\sum_{K \in \mathcal{T}_{H}} \int_{K}\left[\mathcal{A}^{0}\left(x, \nabla v^{H}\left(x_{K}\right)\right)-\mathcal{A}^{0}\left(x_{K}, \nabla v^{H}\left(x_{K}\right)\right)\right] \cdot \nabla w^{H}\left(x_{K}\right) \mathrm{d} x .
$$

The Hölder continuity (4.3) of $\mathcal{A}^{0}(x, \xi)$ in $x,(3.12)$ and the bound (5.4a) then yield the results. 


\subsubsection{Abstract estimates for the HMM upscaling error}

The last term (5.1g) in the error propagation formula (5.1) quantifies the upscaling error. Using $r_{H M M}$ introduced in (4.1), we obtain

$$
\left|\hat{B}^{0}\left(v^{H} ; w^{H}\right)-B^{H}\left(v^{H} ; w^{H}\right)\right| \leq r_{H M M}\left(\nabla v^{H}\right)\left\|\nabla w^{H}\right\|_{L^{p}(\Omega)}, \quad v^{H}, w^{H} \in S_{0}^{1}\left(\Omega, \mathcal{T}_{H}\right) .
$$

Recall that $r_{H M M}\left(\nabla v^{H}\right) \leq r_{\text {mic }}\left(\nabla v^{H}\right)+r_{\text {mod }}\left(\nabla v^{H}\right)$ with $r_{\text {mic }}$ and $r_{\text {mod }}$ from (4.2). While structural assumptions about the spatial heterogeneities of $\mathcal{A}^{\varepsilon}$ are necessary to estimate the modeling error $r_{\text {mod }}$, an abstract error estimate for the micro error $r_{\text {mic }}$ holds for general maps $\mathcal{A}^{\varepsilon}$. Explicit bounds for $r_{\text {mod }}$ and $r_{\text {mic }}$ in the case of $p=2, \alpha=1, \beta=2$ will be derived in Section 5.3.2.

Lemma 5.9. Let $\mathcal{A}^{\varepsilon}$ satisfy $\left(\mathcal{A}_{0-2}\right)$. For both periodic coupling $W\left(K_{\delta}\right)=\mathcal{W}_{\mathrm{per}}^{1, p}\left(K_{\delta}\right)$ or Dirichlet coupling $W\left(K_{\delta}\right)=W_{0}^{1, p}\left(K_{\delta}\right)$ in the micro problems (3.6), the micro error $r_{\mathrm{mic}}\left(\nabla v^{H}\right)$ from (4.2a) is bounded by

$$
\begin{aligned}
r_{\text {mic }}\left(\nabla v^{H}\right) \leq C\left[L_{0}+\right. & \left.\kappa_{1}+\kappa_{2}+\left\|\nabla v^{H}\right\|_{L^{p}(\Omega)}\right]^{p-1-\gamma} \\
& \times\left(\sum_{K \in \mathcal{T}_{H}} \frac{|K|}{\left|K_{\delta}\right|} \inf _{z^{h} \in S^{1}\left(K_{\delta}, \mathcal{T}_{h}\right)}\left\|\nabla \bar{\chi}_{K}^{\nabla v^{H}\left(x_{K}\right)}-\nabla z^{h}\right\|_{L^{p}\left(K_{\delta}\right)}^{p}\right)^{\frac{\gamma}{p}},
\end{aligned}
$$

for any $v^{H} \in S_{0}^{1}\left(\Omega, \mathcal{T}_{H}\right)$, where $\bar{\chi}_{K}^{\xi}$ solves (3.8) and $C$ is independent of $H, h, \delta$ and $\varepsilon$.

Proof. For given $\xi \in \mathbb{R}^{d}$ and $K \in \mathcal{T}_{H}$, let $\bar{\chi}_{K}^{\xi}$ and $\chi_{K}^{\xi, h}$ be the solution of (3.8) and (3.7), respectively. Using $a_{K}^{\xi}$ defined in (3.13) we get from (3.17) and the micro problems (3.14) that for $z^{h} \in S^{1}\left(K_{\delta}, \mathcal{T}_{h}\right)$

$$
\lambda\left\|\nabla \bar{\chi}_{K}^{\xi}-\nabla \chi_{K}^{\xi, h}\right\|_{L^{p}\left(K_{\delta}\right)}^{\beta} \leq \mathcal{R}(\xi, K)^{\beta-p}\left(a_{K}^{\xi}\left(\bar{\chi}_{K}^{\xi} ; \bar{\chi}_{K}^{\xi}-z^{h}\right)-a_{K}^{\xi}\left(\chi_{K}^{\xi, h} ; \bar{\chi}_{K}^{\xi}-z^{h}\right)\right.
$$

with $\mathcal{R}(\xi, K)=\left(\kappa_{2}+2|\xi|\right)\left|K_{\delta}\right|^{1 / p}+\left\|\nabla \bar{\chi}_{K}^{\xi}\right\|_{L^{p}\left(K_{\delta}\right)}+\left\|\nabla \chi_{K}^{\xi, h}\right\|_{L^{p}\left(K_{\delta}\right)}$. Analogously to (3.26) we get

$$
\left\|\nabla \bar{\chi}_{K}^{\xi}-\nabla \chi_{K}^{\xi, h}\right\|_{L^{p}\left(K_{\delta}\right)} \leq C\left[\left(L_{0}+\kappa_{1}+\kappa_{2}+|\xi|\right)\left|K_{\delta}\right|^{\frac{1}{p}}\right]^{\frac{\beta-\alpha-1}{\beta-\alpha}}\left\|\nabla \bar{\chi}_{K}^{\xi}-\nabla z^{h}\right\|_{L^{p}\left(K_{\delta}\right)}^{\frac{1}{\beta-\alpha}} .
$$

Combining this estimate with $\left(\mathcal{A}_{1}\right)$ and $(3.12)$ we obtain for $\overline{\mathcal{A}}_{K}^{0}$ and $\mathcal{A}_{K}^{0, h}$ given in (3.9)

$$
\left|\overline{\mathcal{A}}_{K}^{0}(\xi)-\mathcal{A}_{K}^{0, h}(\xi)\right| \leq C\left[L_{0}+\kappa_{1}+\kappa_{2}+|\xi|\right]^{p-1-\gamma}\left(\frac{1}{\left|K_{\delta}\right|}\left\|\nabla \bar{\chi}_{K}^{\xi}-\nabla z^{h}\right\|_{L^{p}\left(K_{\delta}\right)}^{p}\right)^{\gamma},
$$

where $\gamma=\alpha /(\beta-\alpha)$. Setting $\xi=\nabla v^{H}\left(x_{K}\right)$ for $K \in \mathcal{T}_{H}$ and using (5.8) and (3.12), we obtain the result by noting that $z^{h}$ can be chosen arbitrarily in $S^{1}\left(K_{\delta}, \mathcal{T}_{h}\right)$.

\subsection{Proof of the general convergence result}

In this section, we prove Theorem 4.2. We start by proving the convergence of the micro error.

Lemma 5.10. Assume that $\mathcal{A}^{\varepsilon}$ satisfies $\left(\mathcal{A}_{0-2}\right)$. Let the time step size $\Delta t>0$, the macro mesh size $H>0$, the sampling domain size $\delta>0$ and the coupling conditions for (3.6) be given. Then, for any set $\left\{\mathcal{U}_{n}^{H}\right\}_{1 \leq n \leq N} \subset$ $S_{0}^{1}\left(\Omega, \mathcal{T}_{H}\right)$ with $\sum_{n=0}^{N-1} \Delta t\left\|\nabla \mathcal{U}_{n+1}^{H}\right\|_{L^{p}(\Omega)}^{p}$ bounded independently of the micro mesh size $h$, the micro error $r_{\text {mic }}$ from (4.2a) satisfies

$$
\lim _{h \rightarrow 0}\left(\sum_{n=0}^{N-1} \Delta t r_{\text {mic }}\left(\nabla \mathcal{U}_{n+1}^{H}\right)^{p^{\prime}}\right)^{\frac{1}{p^{\prime}}}=0
$$


Proof. Let $0<\eta<1$ be given. Let $\xi \in \mathbb{R}^{d}, K \in \mathcal{T}_{H}$ and $\bar{\chi}_{K}^{\xi} \in W\left(K_{\delta}\right)$ solving (3.8). First, we observe that for sufficiently small $h$ we have $\inf _{z^{h} \in S^{1}\left(K_{\delta}, \mathcal{T}_{h}\right)}\left\|\bar{\chi}_{K}^{\xi}-z^{h}\right\|_{W\left(K_{\delta}\right)}<\eta$. Indeed, as $C^{\infty}\left(K_{\delta}\right) \cap W\left(K_{\delta}\right)$ is dense in $W\left(K_{\delta}\right)$ (for both periodic and Dirichlet coupling), there exists $z_{\eta} \in C^{\infty}\left(K_{\delta}\right) \cap W\left(K_{\delta}\right)$ such that $\left\|\bar{\chi}_{K}^{\xi}-z_{\eta}\right\|_{W\left(K_{\delta}\right)}<\eta / 2$. Setting $z^{h}=\mathcal{I}_{h} z_{\eta}$ and applying the interpolation estimate (5.4a) to the nodal interpolant $\mathcal{I}_{h}$ on $K_{\delta}$ (for some $p^{*}$ satisfying $p \leq p^{*}$ and $p^{*}>d / 2$ ) we get

$$
\left\|\bar{\chi}_{K}^{\xi}-z^{h}\right\|_{W\left(K_{\delta}\right)} \leq\left\|\bar{\chi}_{K}^{\xi}-z_{\eta}\right\|_{W\left(K_{\delta}\right)}+\left\|z_{\eta}-z^{h}\right\|_{W\left(K_{\delta}\right)}<\frac{\eta}{2}+C h\left\|z_{\eta}\right\|_{W^{2, p^{*}}\left(K_{\delta}\right)},
$$

i.e., in particular, there exists $h_{0}(\eta)>0$ such that $\left\|\bar{\chi}_{K}^{\xi}-z^{h}\right\|_{W\left(K_{\delta}\right)}<\eta$ for all $h \leq h_{0}(\eta)$.

Using this result for $\xi=\nabla \mathcal{U}_{n+1}^{H}\left(x_{K}\right)$ and any $K \in \mathcal{T}_{H}, 0 \leq n \leq N-1$, i.e., a finite set of parameters independent of $h$, we obtain with Lemma 5.9 and (3.12) that

$$
\lim _{h \rightarrow 0}\left(\sum_{n=0}^{N-1} \Delta t r_{\text {mic }}\left(\nabla \mathcal{U}_{n+1}^{H}\right)^{p^{\prime}}\right)^{\frac{1}{p^{\prime}}} \leq C\left[L_{0}+\kappa_{1}+\kappa_{2}+\left(\sum_{n=0}^{N-1} \Delta t\left\|\nabla \mathcal{U}_{n+1}^{H}\right\|_{L^{p}(\Omega)}^{p}\right)^{\frac{1}{p}}\right]^{p-1-\gamma}\left|K_{\delta}\right|^{-\frac{\gamma}{p}} \eta^{\gamma},
$$

and thus, as $0<\eta<1$ can be chosen arbitrarily small, the micro error vanishes.

Proof of Theorem 4.2 .

Step 1. General error bound. Let $\mathcal{U} \in E$ with $\mathcal{U} \in \mathcal{C}^{0}\left([0, T], W^{2, p^{*}}(\Omega)\right), \partial_{t} \mathcal{U} \in \mathcal{C}^{0}\left([0, T], W^{1, p}(\Omega)\right) \cap$ $\mathcal{C}^{0}\left([0, T], W^{2, \tilde{p}}(\Omega)\right)$ and $\partial_{t}^{2} \mathcal{U} \in \mathcal{C}^{0}\left([0, T], L^{2}(\Omega)\right)$ where $\tilde{p}=\min \left\{p^{*}, 2\right\}$ and some $p^{*}$ with $p \leq p^{*}$ and $p^{*}>d / 2$. We then set $\theta_{n}^{H}=u_{n}^{H}-\mathcal{U}_{n}^{H}$ with $\mathcal{U}_{n}^{H}=\mathcal{I}_{H} \mathcal{U}\left(\cdot, t_{n}\right)$ for $0 \leq n \leq N$. Summing (3.21) from $n=0, \ldots, N-1$, using (3.12) and the error propagation formula (5.1) yields

$$
\begin{aligned}
\lambda_{c}^{\frac{p}{\beta}} \sum_{n=0}^{N-1} \Delta t\left\|\nabla \theta_{n+1}^{H}\right\|_{L^{p}(\Omega)}^{p} & \leq \mathcal{R}\left(u_{n}^{H}, \mathcal{U}_{n}^{H}\right)^{\frac{p(\beta-p)}{\beta}}\left(\sum_{n=0}^{N-1} \Delta t\left(B^{H}\left(u_{n+1}^{H} ; \theta_{n+1}^{H}\right)-B^{H}\left(\mathcal{U}_{n+1}^{H} ; \theta_{n+1}^{H}\right)\right)\right)^{\frac{p}{\beta}} \\
& =\mathcal{R}\left(u_{n}^{H}, \mathcal{U}_{n}^{H}\right)^{\frac{p(\beta-p)}{\beta}}\left(-\sum_{n=0}^{N-1} \Delta t \int_{\Omega} \bar{\partial}_{t} \theta_{n}^{H} \theta_{n+1}^{H} \mathrm{~d} x+\sum_{n=0}^{N-1} \mathcal{B}_{n}^{t o t}\right)^{\frac{p}{\beta}}
\end{aligned}
$$

where $\mathcal{B}_{n}^{\text {tot }}$ denotes the sum of the terms (5.1a)-(5.1g) with test function $\theta_{n+1}^{H}$ and $\mathcal{R}\left(u_{n}^{H}, \mathcal{U}_{n}^{H}\right)$ is given by

$$
\mathcal{R}\left(u_{n}^{H}, \mathcal{U}_{n}^{H}\right)=\left(L_{0}+\kappa_{1}+\kappa_{2}\right)(T|\Omega|)^{\frac{1}{p}}+\left(\sum_{n=0}^{N-1} \Delta t\left\|\nabla u_{n+1}^{H}\right\|_{L^{p}(\Omega)}^{p}\right)^{\frac{1}{p}}+\left(\sum_{n=0}^{N-1} \Delta t\left\|\nabla \mathcal{U}_{n+1}^{H}\right\|_{L^{p}(\Omega)}^{p}\right)^{\frac{1}{p}} .
$$

Using the bound (3.29) analogously for $\theta_{n}^{H}$, estimating the terms (5.1a)-(5.1g) by Lemmas 5.1, 5.2, 5.3, 5.5, 5.7, 5.8 and inequality (5.6) and using (3.12) we obtain

$$
\begin{aligned}
\lambda_{c}^{\frac{p}{\beta}} \sum_{n=0}^{N-1} \Delta & t\left\|\nabla \theta_{n+1}^{H}\right\|_{L^{p}(\Omega)}^{p} \leq \mathcal{R}\left(u_{n}^{H}, \mathcal{U}_{n}^{H}\right)^{\frac{p(\beta-p)}{\beta}}\left[-\frac{1}{2}\left\|\theta_{N}^{H}\right\|_{L^{2}(\Omega)}^{2}+\frac{1}{2}\left\|\theta_{0}^{H}\right\|_{L^{2}(\Omega)}^{2}\right. \\
& +C\left\{\left\|u^{0}-\mathcal{U}\right\|_{E}+\left[\tilde{\kappa}_{1}+\left\|u^{0}\right\|_{E}+\|\mathcal{U}\|_{E}\right]^{p-1-\gamma}\left\|u^{0}-\mathcal{U}\right\|_{E}^{\gamma}\right. \\
& +\left[\left\|\partial_{t} \mathcal{U}\right\|_{\mathcal{C}^{0}\left([0, T], W^{2, \tilde{p}}(\Omega)\right)}+\left\|\partial_{t}^{2} \mathcal{U}\right\|_{\mathcal{C}^{0}\left([0, T], L^{2}(\Omega)\right)}\right](\Delta t+H) \\
& +\left[L_{0}+\kappa_{1}+\|\mathcal{U}\|_{\mathcal{C}^{0}\left([0, T], W^{2, p^{*}}(\Omega)\right)}+\left\|\partial_{t} \mathcal{U}\right\|_{\mathcal{C}^{0}\left([0, T], W^{1, p}(\Omega)\right)}\right]^{p-1}\left(\Delta t^{\gamma}+H^{\gamma}+H^{\tilde{\gamma}}\right) \\
& \left.\left.+\left(\sum_{n=0}^{N-1} \Delta t r_{H M M}\left(\nabla \mathcal{U}_{n+1}^{H}\right)^{p^{\prime}}\right)^{\frac{1}{p^{\prime}}}\right\}\left(\sum_{n=0}^{N-1} \Delta t\left\|\nabla \theta_{n+1}^{H}\right\|_{L^{p}(\Omega)}^{p}\right)^{\frac{1}{p}}\right]^{\frac{p}{\beta}},
\end{aligned}
$$


For the rest of the proof, the term $-\frac{1}{2}\left\|\theta_{N}^{H}\right\|_{L^{2}(\Omega)}^{2} \leq 0$ in (5.11a) can be omitted.

Step 2. Density in $E$. Let $0<\eta<1$ be given. To show the convergence of (5.11) we choose $\mathcal{U} \in C^{\infty}(\bar{\Omega} \times[0, T])$ such that $\mathcal{U}(\cdot, t) \in C_{0}^{\infty}(\Omega)$ for any $t \in[0, T]$ and $\left\|u^{0}-\mathcal{U}\right\|_{E}<\eta / 2$. This is possible as the polynomials $\sum_{i} t^{i} v_{i}$ with $v_{i} \in W_{0}^{1, p}(\Omega)$ are dense in $E$ (see [52], Prop. 23.23), and $C_{0}^{\infty}(\Omega)$ is dense in $W_{0}^{1, p}(\Omega)$. Note that such $\mathcal{U}$ further satisfies $\|\mathcal{U}\|_{E}<\left\|u^{0}\right\|_{E}+1 / 2$.

We then set $\mathcal{U}_{n}^{H}=\mathcal{I}_{H} \mathcal{U}\left(\cdot, t_{n}\right)$ as above in (5.11). Using the interpolation estimate (5.4a) in space and a bound analogous to (5.3) in time we get that for $s \in\left[t_{n}, t_{n+1}\right]$ and $0 \leq n \leq N-1$

$$
\left\|\nabla \mathcal{U}(\cdot, s)-\nabla \mathcal{U}_{n+1}^{H}\right\|_{L^{p}(\Omega)} \leq C(\Delta t+H)\left(\|\mathcal{U}\|_{\mathcal{C}^{0}\left([0, T], W^{\left.2, p^{*}(\Omega)\right)}\right.}+\left\|\partial_{t} \nabla \mathcal{U}\right\|_{\mathcal{C}^{0}\left([0, T], L^{p}(\Omega)\right)}\right) .
$$

Hence, combining that with the properties of $\mathcal{U}$, there exists $D_{0}(\eta)>0$ such that for all $\Delta t, H \leq D_{0}(\eta)$

$$
\left(\sum_{n=0}^{N-1} \int_{t_{n}}^{t_{n+1}}\left\|\nabla u^{0}(\cdot, t)-\nabla \mathcal{U}_{n+1}^{H}\right\|_{L^{p}(\Omega)}^{p} \mathrm{~d} t\right)^{\frac{1}{p}}<\eta, \quad\left(\sum_{n=0}^{N-1} \Delta t\left\|\nabla \mathcal{U}_{n+1}^{H}\right\|_{L^{p}(\Omega)}^{p}\right)^{1 / p} \leq\left\|u^{0}\right\|_{E}+1 .
$$

Further, by possibly taking a smaller value for $D_{0}(\eta)$ we simultaneously get that for $H \leq D_{0}(\eta)$

$$
\max _{1 \leq n \leq N}\left\|u^{0}\left(\cdot, t_{n}\right)-\mathcal{U}_{n}^{H}\right\|_{L^{2}(\Omega)} \leq C_{E}\left\|u^{0}-\mathcal{U}\right\|_{E}+C H\|\mathcal{U}\|_{\mathcal{C}^{0}\left([0, T], W^{2, p^{*}}(\Omega)\right)}<C_{E} \frac{\eta}{2}+\frac{\eta}{2},
$$

where we used the embeddings $E \hookrightarrow \mathcal{C}^{0}\left([0, T], L^{2}(\Omega)\right.$ ) (with operator norm $\left.C_{E}\right), W^{1, p}(\Omega) \hookrightarrow L^{2}(\Omega)$ and the interpolation estimate (5.4a).

Step 3. Bound for $\mathcal{R}$. Next, we bound $\mathcal{R}\left(u_{n}^{H}, \mathcal{U}_{n}^{H}\right)$ from (5.10). From Theorem 3.8 and the convergence $\| g-$ $u_{0}^{H} \|_{L^{2}(\Omega)} \rightarrow 0$ for $H \rightarrow 0$ we have that there exists $H_{0}>0$ such that for all $H \leq H_{0}$ it holds

$$
\left(\sum_{n=0}^{N-1} \Delta t\left\|\nabla u_{n+1}^{H}\right\|_{L^{p}(\Omega)}^{p}\right)^{\frac{1}{p}} \leq C\left(\left(L_{0}+\kappa_{1}+\kappa_{2}\right)+\|f\|_{L^{p^{\prime}}(\Omega)}^{\frac{1}{p-1}}+\|g\|_{L^{2}(\Omega)}^{\frac{2}{p}}+1\right),
$$

i.e., independent of the initial approximation $u_{0}^{H}$. Combining that with (5.12) we get that $\mathcal{R}\left(u_{n}^{H}, \mathcal{U}_{n}^{H}\right)$ is bounded independently of $\mathcal{U}, \eta, \Delta t$ and $H$ for all $\Delta t, H \leq \min \left\{D_{0}(\eta), H_{0}\right\}$. Additionally, the same bound is valid for the last term in (5.11e) as $\left(\sum_{n=0}^{N-1} \Delta t\left\|\nabla \theta_{n+1}^{H}\right\|_{L^{p}(\Omega)}^{p}\right)^{1 / p} \leq \mathcal{R}\left(u_{n}^{H}, \mathcal{U}_{n}^{H}\right)$.

Step 4. Convergence of HMM error. By decomposing $r_{H M M}$ in (5.11e) into micro error $r_{\text {mic }}$ and modeling error $r_{\text {mod }}$, see (4.2), we obtain from Lemma 5.10 and the convergence of the modeling error assumed in Theorem 4.2 that $\lim _{r_{\text {mod }} \rightarrow 0} \lim _{h \rightarrow 0}\left(\sum_{n=0}^{N-1} \Delta t r_{H M M}\left(\nabla \mathcal{U}_{n+1}^{H}\right)^{p^{\prime}}\right)^{1 / p^{\prime}}=0$ for given $\Delta t$ and $H$.

Step 5. Convergence in $L^{p}\left(W^{1, p}\right)$ norm. First using the embedding $E \hookrightarrow \mathcal{C}^{0}\left([0, T], L^{2}(\Omega)\right)$ (again with operator norm $C_{E}$ ) and the interpolation estimate (5.4a) the initial error $\theta_{0}^{H}$ in (5.11a) can be bounded by

$$
\left\|\theta_{0}^{H}\right\|_{L^{2}(\Omega)} \leq\left\|g-u_{0}^{H}\right\|_{L^{2}(\Omega)}+C_{E}\left\|u^{0}-\mathcal{U}\right\|_{E}+C H\|\mathcal{U}\|_{\mathcal{C}^{0}\left([0, T], W^{2, p^{*}}(\Omega)\right)} .
$$

Combining then (5.11) with (5.14) and using the properties of $\mathcal{U}$ derived in Step 2 and 3, there exists $0<$ $D_{1}(\eta) \leq \min \left\{D_{0}(\eta), H_{0}\right\}$ such that for $\Delta t, H \leq D_{1}(\eta)$

$$
\lim _{r_{\bmod } \rightarrow 0} \lim _{h \rightarrow 0}\left(\sum_{n=0}^{N-1} \Delta t\left\|\nabla \theta_{n+1}^{H}\right\|_{L^{p}(\Omega)}^{p}\right)^{\frac{1}{p}} \leq C\left(\eta+\eta^{\gamma}\right)
$$

where $C$ is independent of $\eta, \mathcal{U}$ as well as $H, \Delta t, \delta$ and $h$. The convergence in the discrete $L^{p}\left(W^{1, p}\right)$ norm then follows from (5.15) and (5.12) as $\eta$ is arbitrarily small. 
Step 6. Convergence in $\mathcal{C}^{0}\left(L^{2}\right)$ norm. Let $1 \leq K \leq N$. Summing the error formula (5.1) with $w^{H}=\theta_{n+1}^{H}$ for $n=0, \ldots, K-1$, using (3.29) and the monotonicity of $B^{H}$ from Lemma 3.5 we get

$$
\frac{1}{2}\left\|\theta_{K}^{H}\right\|_{L^{2}(\Omega)}^{2}-\frac{1}{2}\left\|\theta_{0}^{H}\right\|_{L^{2}(\Omega)}^{2} \leq \sum_{n=0}^{K-1} \Delta t\left[\int_{\Omega} \bar{\partial}_{t} \theta_{n}^{H} \theta_{n+1}^{H} \mathrm{~d} x+B^{H}\left(u_{n+1}^{H} ; \theta_{n+1}^{H}\right)-B^{H}\left(\mathcal{U}_{n+1}^{H} ; \theta_{n+1}^{H}\right)\right],
$$

which can be bounded by $\sum_{n=0}^{K-1} \mathcal{B}_{n}^{\text {tot }}$ with $\mathcal{B}_{n}^{\text {tot }}$ used in (5.9). Then, analogously to Step 5, estimating $\mathcal{B}_{n}^{\text {tot }}$ like in (5.11) and combining that with (5.13) proves the convergence of $\max _{1 \leq n \leq N}\left\|u^{0}\left(\cdot, t_{n}\right)-u_{n}^{H}\right\|_{L^{2}(\Omega)}$.

\subsection{Proof of the explicit convergence rates for strongly monotone and Lipschitz maps}

In this section, the fully discrete a priori error estimates for $p=2$ and $\alpha=1, \beta=2$ in $\left(\mathcal{A}_{1-2}\right)$ are shown. The estimates of the temporal and macro spatial error from Theorem 4.4 are proved in two steps: the $L^{2}\left(H^{1}\right)$ estimate is proved below using the results of Sections 5.1.2 and 5.2 and then, in Section 5.3.1, the $\mathcal{C}^{0}\left(L^{2}\right)$ bound is derived using an elliptic projection. Finally, we show the estimates of micro and modeling error from Theorems 4.5 and 4.6 in Section 5.3.2.

Proof of $L^{2}\left(H^{1}\right)$ estimate from Theorem 4.4. Recall that $\theta_{n}^{H}=u_{n}^{H}-\mathcal{U}_{n}^{H}$ for $0 \leq n \leq N$. To derive the $L^{2}\left(H^{1}\right)$ estimate we set $\mathcal{U}_{n}^{H}=\mathcal{I}_{H} u^{0}\left(\cdot, t_{n}\right)$. Note, that when deriving the error propagation formula (5.1) for $\mathcal{U}=u^{0}$ with the regularity of $u^{0}$ assumed in Theorem 4.4 (with $\mu=1$ ), one can use

$$
\int_{\Omega} \partial_{t} u^{0}(x, t) w \mathrm{~d} x+B^{0}\left(u^{0}(\cdot, t) ; w\right)=\int_{\Omega} f w \mathrm{~d} x, \quad \forall w \in W_{0}^{1, p}(\Omega), \forall t \in(0, T],
$$

instead of the weak formulation in time. Hence, the error terms (5.1a) and (5.1b) vanish. Then, analogously to (5.11), we estimate (5.1c)-(5.1g) using Lemmas 5.3, 5.5, 5.7, 5.8 and (5.6). Observing that $\gamma=\alpha /(\beta-\alpha)=1$, $p / \beta=1$ and that (4.4b) for $\mu=1$ yields the Hölder continuity (4.3) with $\tilde{\gamma}=1$, we get

$$
\begin{aligned}
\frac{1}{2}\left\|\theta_{N}^{H}\right\|_{L^{2}(\Omega)}^{2} & +\lambda_{c} \sum_{n=0}^{N-1} \Delta t\left\|\nabla \theta_{n+1}^{H}\right\|_{L^{2}(\Omega)}^{2} \\
\leq & \frac{1}{2}\left\|\theta_{0}^{H}\right\|_{L^{2}(\Omega)}^{2}+C\left(\Delta t+H+\max _{1 \leq n \leq N} r_{H M M}\left(\nabla \mathcal{U}_{n}^{H}\right)\right)\left(\sum_{n=0}^{N-1} \Delta t\left\|\nabla \theta_{n+1}^{H}\right\|_{L^{2}(\Omega)}^{2}\right)^{\frac{1}{2}} \\
\leq & \frac{1}{2}\left\|\theta_{0}^{H}\right\|_{L^{2}(\Omega)}^{2}+C\left(\Delta t+H+\max _{1 \leq n \leq N} r_{H M M}\left(\nabla \mathcal{U}_{n}^{H}\right)\right)^{2}+\frac{\lambda_{c}}{2} \sum_{n=0}^{N-1} \Delta t\left\|\nabla \theta_{n+1}^{H}\right\|_{L^{2}(\Omega)}^{2},
\end{aligned}
$$

which combined with $(5.4 \mathrm{a})$ concludes the proof for $\mu=1$.

\subsubsection{Optimal bounds of macro error in spatial $L^{2}$ norm}

To obtain quadratic convergence of the macro spatial error in the $\mathcal{C}^{0}\left(L^{2}\right)$ norm we introduce an elliptic projection of the homogenized solution $u^{0}$. We derive its approximation properties and improve the bounds of (5.1d), (5.1e) and (5.1f) to get the sharp bounds of Theorem 4.4.

Remark 5.11. Let $\mathcal{A}: \Omega \times \mathbb{R}^{d} \rightarrow \mathbb{R}^{d}$ with $\mathcal{A}(x, \cdot) \in \mathcal{C}^{1}\left(\mathbb{R}^{d} ; \mathbb{R}^{d}\right)$ for a.e. $x \in \Omega$. Then, the map $\mathcal{A}$ satisfies hypotheses $\left(\mathcal{A}_{1-2}\right)$ with $p=2, \alpha=1, \beta=2$ if and only if $D_{\xi} \mathcal{A}(x, \xi)$ is uniformly elliptic and bounded

$$
D_{\xi} \mathcal{A}(x, \xi) \eta \cdot \eta \geq \lambda|\eta|^{2}, \quad\left|D_{\xi} \mathcal{A}(x, \xi) \eta\right| \leq L|\eta|, \quad \forall \xi, \eta \in \mathbb{R}^{d} \text {, a.e. } x \in \Omega .
$$

Further, for $\xi, \eta \in \mathbb{R}^{d}$ and a.e. $x \in \Omega$, we have the identity

$$
\mathcal{A}(x, \xi+\eta)=\mathcal{A}(x, \xi)+D_{\xi} \mathcal{A}(x, \xi) \eta+\int_{0}^{1} D_{\xi} \mathcal{A}(x, \xi+\tau \eta)-D_{\xi} \mathcal{A}(x, \xi) \mathrm{d} \tau \eta
$$


If additionally $D_{\xi} \mathcal{A}(x, \cdot)$ is Lipschitz continuous $\left(e . g ., \mathcal{A}(x, \cdot) \in W^{2, \infty}\left(\mathbb{R}^{d} ; \mathbb{R}^{d}\right)\right)$, we obtain from $(5.17)$

$$
\left|\int_{\Omega}\left[\mathcal{A}(x, \nabla v+\nabla w)-\mathcal{A}(x, \nabla v)-D_{\xi} \mathcal{A}(x, \nabla v) \cdot \nabla w\right] \cdot \nabla z \mathrm{~d} x\right| \leq L_{\mathcal{A}}\|w\|_{W^{1,4}(\Omega)}^{2}\|z\|_{H^{1}(\Omega)},
$$

for $v, w \in W^{1,4}(\Omega), z \in H^{1}(\Omega)$ and where $L_{\mathcal{A}}=\operatorname{ess}_{\sup _{x \in \Omega}} L_{x}$ with Lipschitz constant $L_{x}$ of $\mathcal{A}(x, \cdot)$.

Elliptic projection. Let $u^{0}(x, t) \in E$ be the exact solution of the homogenized problem (2.3). The elliptic projection $\tilde{u}^{H, 0}(\cdot, t)$ of $u^{0}(\cdot, t)$ is given by the variational problem: find $\tilde{u}^{H, 0}(\cdot, t) \in S_{0}^{1}\left(\Omega, \mathcal{T}_{H}\right)$ such that

$$
B_{\pi}\left(t ; \tilde{u}^{H, 0}(\cdot, t), w^{H}\right)=B_{\pi}\left(t ; u^{0}(\cdot, t), w^{H}\right), \quad \forall w^{H} \in S_{0}^{1}\left(\Omega, \mathcal{T}_{H}\right),
$$

where, for a.e. $t \in(0, T)$, the bilinear form $B_{\pi}$ is defined as, for $v, w \in H_{0}^{1}(\Omega)$,

$$
B_{\pi}(t ; v, w)=\int_{\Omega} \mathscr{A}^{0}(x, t) \nabla v \cdot \nabla w \mathrm{~d} x, \quad \text { with } \mathscr{A}^{0}(x, t)=D_{\xi} \mathcal{A}^{0}\left(x, \nabla u^{0}(x, t)\right),
$$

for a.e. $(x, t) \in \Omega \times(0, T)$. The existence and uniqueness of the elliptic projection $\tilde{u}^{H, 0}(\cdot, t)$ defined in $(5.19)$ is studied in Lemma 5.12. Note that for a linear problem we recover the tensor $\mathscr{A}^{0}(x, t)=a^{0}(x, t)$ and $\partial_{t} \mathscr{A}^{0}(x, t)=$ $\partial_{t} a^{0}(x, t)$ as considered in $[8,43,49]$.

Lemma 5.12. Let $p=2$ and assume that $\mathcal{A}^{0}$ satisfies $\left(\mathcal{A}_{1-2}\right)$ with $\alpha=1, \beta=2$ and $\mathcal{A}^{0}(x, \cdot) \in \mathcal{C}^{1}\left(\mathbb{R}^{d} ; \mathbb{R}^{d}\right)$ for a.e. $x \in \Omega$. If the homogenized solution $u^{0}$ satisfies $u^{0} \in L^{2}\left(0, T ; H_{0}^{1}(\Omega)\right)$, then, for a.e. $t \in(0, T)$, the bilinear form $B_{\pi}$ given by (5.20) is uniformly elliptic and bounded and there exists a unique solution $\tilde{u}^{H, 0}(\cdot, t)$ to $(5.19)$. Further, we have

$$
\left\|\nabla \tilde{u}^{H, 0}(\cdot, t)\right\|_{L^{2}(\Omega)} \leq \frac{L}{\lambda}\left\|\nabla u^{0}(\cdot, t)\right\|_{L^{2}(\Omega)}, \quad \text { a.e. } t \in(0, T)
$$

Proof. Due to Remark 5.11 and the regularity of $u^{0}$ the tensor $\mathscr{A}^{0}$ satisfies $\mathscr{A}_{i j}^{0} \in L^{\infty}\left(0, T ; L^{\infty}(\Omega)\right)$, for $1 \leq$ $i, j \leq d$. Using again Remark 5.11 we have for $v, w \in H_{0}^{1}(\Omega)$ that $\left|B_{\pi}(t ; v, w)\right| \leq L\|\nabla v\|_{L^{2}(\Omega)}\|\nabla w\|_{L^{2}(\Omega)}$, $\lambda\|\nabla v\|_{L^{2}(\Omega)}^{2} \leq B_{\pi}(t ; v, v)$ for a.e. $t \in(0, T)$. Thus, the Lax-Milgram theorem concludes the proof.

Lemma 5.13. Let $p=2$ and assume that $\mathcal{A}^{0}$ satisfies $\left(\mathcal{A}_{1-2}\right)$ with $\alpha=1, \beta=2$ and $\mathcal{A}^{0}(x, \cdot) \in \mathcal{C}^{1}\left(\mathbb{R}^{d} ; \mathbb{R}^{d}\right)$ for a.e. $x \in \Omega$. Let the homogenized solution $u^{0}$ and $\mathscr{A}^{0}$ defined in (5.20) satisfy

$$
u^{0}, \partial_{t} u^{0} \in \mathcal{C}^{0}\left([0, T], H_{0}^{1}(\Omega)\right), \quad \mathscr{A}_{i j}^{0}, \partial_{t} \mathscr{A}_{i j}^{0} \in \mathcal{C}^{0}\left([0, T], L^{\infty}(\Omega)\right), \quad \text { for } 1 \leq i, j \leq d .
$$

Then, the map $t \mapsto \tilde{u}^{H, 0}(\cdot, t) \in S_{0}^{1}\left(\Omega, \mathcal{T}_{H}\right)$, where $\tilde{u}^{H, 0}$ is the elliptic projection (5.19), is of class $\mathcal{C}^{1}$.

Proof. As (5.19) defines a linear elliptic projection, the proof is based on similar arguments as given in ([8], Eq. (5.3)). A full proof is available in ([36], Lem. 6.5.4).

Lemma 5.14. Let $p=2$ and assume that $\mathcal{A}^{0}$ satisfies $\left(\mathcal{A}_{1-2}\right)$ with $\alpha=1, \beta=2$ and $\mathcal{A}^{0}(x, \cdot) \in \mathcal{C}^{1}\left(\mathbb{R}^{d} ; \mathbb{R}^{d}\right)$ for a.e. $x \in \Omega$. Let $u^{0}$ be the solution of the homogenized problem (2.3), $\tilde{u}^{H, 0}$ its elliptic projection (5.19) and $\mathscr{A}^{0}$ the tensor given by (5.20). Let $k \in\{1,2\}$ and assume

$$
u^{0}, \partial_{t} u^{0} \in \mathcal{C}^{0}\left([0, T], H^{2}(\Omega)\right), \quad \mathscr{A}_{i j}^{0}, \partial_{t} \mathscr{A}_{i j}^{0} \in \mathcal{C}^{0}\left([0, T], W^{k-1, \infty}(\Omega)\right), \quad \text { for } 1 \leq i, j \leq d .
$$

Then, for any $t \in[0, T]$, we have the error estimates

$$
\begin{aligned}
\left\|\tilde{u}^{H, 0}(\cdot, t)-u^{0}(\cdot, t)\right\|_{H^{1}(\Omega)} & \leq C H, \\
\left\|\tilde{u}^{H, 0}(\cdot, t)-u^{0}(\cdot, t)\right\|_{L^{2}(\Omega)} & \leq C H^{k},
\end{aligned}
$$

where $C$ is independent of $H$. 
Proof. Due to the linearity of the variational problem (5.19), the proof follows along the lines of the proof of ([8], Lem. 5.1). For a detailed proof we refer to ([36], Lem. 6.5).

To prove the optimal convergence in the $\mathcal{C}^{0}\left(L^{2}\right)$ norm, we further need an estimate for $\| \tilde{u}^{H, 0}(\cdot, t)-$ $u^{0}(\cdot, t) \|_{W^{1, \infty}(\Omega)}$.

Lemma 5.15. Let $p=2$, assume that $\mathcal{A}^{0}$ satisfies $\left(\mathcal{A}_{1-2}\right)$ with $\alpha=1, \beta=2$ and $\mathcal{A}^{0}(x, \cdot) \in \mathcal{C}^{1}\left(\mathbb{R}^{d} ; \mathbb{R}^{d}\right)$ for a.e. $x \in \Omega$. Let $u^{0}$ be the solution of the homogenized problem (2.3), $\tilde{u}^{H, 0}$ its elliptic projection $(5.19), \mathscr{A}^{0}(x, t)$ be given by $(5.20)$ and $u^{0, *}(\cdot, t) \in H_{0}^{1}(\Omega)$ be solving the dual problem $B_{\pi}\left(t ; w, u^{0, *}(\cdot, t)\right)=B_{\pi}\left(t ; u^{0}(\cdot, t), w\right)$ for all $w \in H_{0}^{1}(\Omega)$. Assume

$$
u^{0} \in \mathcal{C}^{0}\left([0, T], W^{2, \infty}(\Omega)\right), \quad \mathscr{A}_{i j}^{0} \in \mathcal{C}^{0}\left([0, T], W^{1, \infty}(\Omega)\right), \quad 1 \leq i, j \leq d,
$$

and the "elliptic regularity", for $t \in[0, T]$ and $1<q<\sigma$ with some $\sigma>d$,

$$
\left\|u^{0}(\cdot, t)\right\|_{W^{2, q}(\Omega)}+\left\|u^{0, *}(\cdot, t)\right\|_{W^{2, q}(\Omega)} \leq C\left\|\operatorname{div}\left(\mathscr{A}^{0}(\cdot, t) \nabla u^{0}(\cdot, t)\right)\right\|_{L^{q}(\Omega)} .
$$

If $\left\{\mathcal{T}_{H}\right\}_{H>0}$ is a family of quasi-uniform meshes (e.g., see [16], condition (3.2.28)), then there exists an $H_{0}>0$ such that for every $t \in[0, T]$ and $H<H_{0}$

$$
\left\|\tilde{u}^{H, 0}(\cdot, t)\right\|_{W^{1, \infty}(\Omega)} \leq C\left\|u^{0}(\cdot, t)\right\|_{W^{1, \infty}(\Omega)},\left\|u^{0}(\cdot, t)-\tilde{u}^{H, 0}(\cdot, t)\right\|_{W^{1, \infty}(\Omega)} \leq C H\left\|u^{0}(\cdot, t)\right\|_{W^{2, \infty}(\Omega)},
$$

where $C$ is independent of $H$.

Proof. Recall that the elliptic projection $\tilde{u}^{H, 0}$ is the finite element solution to a linear elliptic problem, see (5.19). The maximum norm error estimates provided by ([14], Thm. 8.1.11 and Cor. 8.1.12) thus apply.

Optimal estimates of macro spatial error using elliptic projection. By adapting Lemmas 5.5 and 5.7 for $\mathcal{U}=u^{0}$ and $\mathcal{U}^{H}$ given by the elliptic projection $\tilde{u}^{H, 0}$ from (5.19) we get the improved bounds (with respect to the macro mesh size $H$ ) given below in Lemmas 5.16 and 5.17.

Lemma 5.16. Let $p=2$, assume that $\mathcal{A}^{0}$ satisfies $\left(\mathcal{A}_{0-2}\right)$ with $\alpha=1, \beta=2$ and $\mathcal{A}^{0}(x, \cdot) \in \mathcal{C}^{1}\left(\mathbb{R}^{d} ; \mathbb{R}^{d}\right)$ for a.e. $x \in \Omega$. Let $u^{0}$ be the solution of the homogenized problem (1.1) and $\tilde{u}^{H, 0}$ be its elliptic projection (5.19). If $u^{0}, \partial_{t} u^{0} \in \mathcal{C}^{0}\left([0, T], H^{2}(\Omega)\right)$ and $\mathscr{A}^{0}$ from (5.20) satisfies (4.5b), then for $\mathcal{U}_{n}^{H}=\tilde{u}_{n}^{H, 0}$ we get

$$
\left|\int_{\Omega}\left[\bar{\partial}_{t} u^{0}\left(x, t_{n}\right)-\bar{\partial}_{t} \mathcal{U}_{n}^{H}\right] w^{H} \mathrm{~d} x\right| \leq C H^{2}\left(\left\|u^{0}\right\|_{\mathcal{C}^{0}\left([0, T], H^{2}(\Omega)\right)}+\left\|\partial_{t} u^{0}\right\|_{\mathcal{C}^{0}\left([0, T], H^{2}(\Omega)\right)}\right)\left\|w^{H}\right\|_{L^{2}(\Omega)},
$$

for $0 \leq n \leq N-1$ and every $w^{H} \in S_{0}^{1}\left(\Omega, \mathcal{T}_{H}\right)$ with a constant $C$ independent of $\Delta t$ and $H$.

Proof. As $\tilde{u}^{H, 0}, \partial_{t} \tilde{u}^{H, 0} \in \mathcal{C}^{0}\left([0, T], S_{0}^{1}\left(\Omega, \mathcal{T}_{H}\right)\right)$, see Lemma 5.13, the bound (5.5) holds analogously for $\mathcal{U}^{H}(x, s)$ given by the elliptic projection (5.19) and using Lemma 5.14 concludes the proof.

While in term (5.1d) optimal quadratic convergence $H^{2}$ for $p=2$ can be obtained even for $\mathcal{U}_{n}^{H}=\mathcal{I}_{H} u^{0}\left(x, t_{n}\right)$, see Remark 5.6, the optimal convergence rate for term (5.1e) is only obtained for $\mathcal{U}_{n}^{H}=\tilde{u}_{n}^{H, 0}$ (due to its particular definition (5.19)).

Lemma 5.17. Let $p=2$ and assume that $\mathcal{A}^{0}$ satisfies $\left(\mathcal{A}_{1-2}\right)$ with $\alpha=1, \beta=2$. Let $u^{0}$ be the solution of the homogenized problem (2.3), $\tilde{u}^{H, 0}$ its elliptic projection (5.19) and $B^{0}$ be given by (3.10). Assume hypotheses (4.5a) for $u^{0}$ and $\mathcal{A}^{0}$, quasi-uniformity and elliptic regularity $(4.5 \mathrm{c})$ as well as regularity $\mathscr{A}_{i j}^{0} \in \mathcal{C}^{0}\left([0, T], W^{1, \infty}(\Omega)\right)$ (for $1 \leq i, j \leq d$ ) with $\mathscr{A}^{0}$ given in (5.20). Then, for $\mathcal{U}_{n+1}^{H}=\tilde{u}_{n+1}^{H, 0}, w^{H} \in S_{0}^{1}\left(\Omega, \mathcal{T}_{H}\right)$ and $0 \leq n \leq N-1$, there exists an $H_{0}>0$ such that for all $H<H_{0}$ we have

$$
\left|B^{0}\left(u^{0}\left(\cdot, t_{n+1}\right) ; w^{H}\right)-B^{0}\left(\mathcal{U}_{n+1}^{H} ; w^{H}\right)\right| \leq C L_{\mathcal{A}^{0}} H^{2}\left\|u^{0}\right\|_{\mathcal{C}^{0}\left([0, T], W^{2, \infty}(\Omega)\right)}^{2}\left\|\nabla w^{H}\right\|_{L^{2}(\Omega)},
$$

where $L_{\mathcal{A}^{0}}=\operatorname{ess}_{\sup _{x \in \Omega}}\left\|D_{\xi} \mathcal{A}^{0}(x, \cdot)\right\|_{W^{1, \infty}\left(\mathbb{R}^{d} ; \mathbb{R}^{d \times d}\right)}$ and $C$ is independent of $\Delta t$ and $H$. 
Proof. Using the Taylor formula (5.17) and the definition of the elliptic projection (5.19) we derive

$$
\begin{aligned}
B^{0}\left(u^{0}\left(\cdot, t_{n+1}\right) ; w^{H}\right)-B^{0}\left(\tilde{u}_{n+1}^{H, 0} ; w^{H}\right)=\int_{\Omega}\left[\mathcal{A}^{0}\left(x, \nabla u^{0}\left(x, t_{n+1}\right)\right)-\mathcal{A}^{0}\left(x, \nabla \tilde{u}_{n+1}^{H, 0}\right)\right] \cdot \nabla w^{H} \mathrm{~d} x \\
=\underbrace{\int_{\Omega} D_{\xi} \mathcal{A}^{0}\left(x, \nabla u^{0}\left(x, t_{n+1}\right)\right)\left(\nabla \tilde{u}_{n+1}^{H, 0}-\nabla u^{0}\left(x, t_{n+1}\right)\right) \cdot \nabla w^{H} d x}_{=B_{\pi}\left(t_{n+1} ; \tilde{u}_{n+1}^{H, 0}-u^{0}\left(\cdot, t_{n+1}\right), w^{H}\right)=0} \\
\quad+\int_{\Omega} \int_{0}^{1}\left\{D_{\xi} \mathcal{A}^{0}\left(x, \nabla u^{0}\left(x, t_{n+1}\right)+\tau\left[\nabla \tilde{u}_{n+1}^{H, 0}-\nabla u^{0}\left(x, t_{n+1}\right)\right]\right)\right. \\
\left.-D_{\xi} \mathcal{A}^{0}\left(x, \nabla u^{0}\left(x, t_{n+1}\right)\right)\right\} \mathrm{d} \tau\left[\nabla \tilde{u}_{n+1}^{H, 0}-\nabla u^{0}\left(x, t_{n+1}\right)\right] \cdot \nabla w^{H} \mathrm{~d} x \\
\leq L_{\mathcal{A}^{0}}\left\|u^{0}\left(\cdot, t_{n+1}\right)-\tilde{u}_{n+1}^{H, 0}\right\|_{W^{1,4}(\Omega)}^{2}\left\|\nabla w^{H}\right\|_{L^{2}(\Omega)},
\end{aligned}
$$

where we used the estimate (5.18). The maximum norm bounds of Lemma 5.15 conclude the proof.

Quadrature error for smooth $\mathcal{A}^{0}$. Finally, a quadratic convergence $H^{2}$ can as well be obtained for the quadrature error (5.1f) when higher regularity of $\mathcal{A}^{0}$ is available.

Lemma 5.18. Let $p=2$. Assume that $\mathcal{A}^{0}$ satisfies the hypothesis (4.4b) for $\mu=2$. Let $B^{0}$ and $\hat{B}^{0}$ be given by (3.10) and (3.11), respectively. Then, the error due to the quadrature (3.2) is bounded by

$$
\left|B^{0}\left(v^{H} ; w^{H}\right)-\hat{B}^{0}\left(v^{H} ; w^{H}\right)\right| \leq C H^{2}\left(L_{0}+\left\|\nabla v^{H}\right\|_{L^{2}(\Omega)}\right)\left\|\nabla w^{H}\right\|_{L^{2}(\Omega)},
$$

for any $v^{H}, w^{H} \in S_{0}^{1}\left(\Omega, \mathcal{T}_{H}\right)$ and where $C$ is independent of $H$.

Proof. An application of ([17], Thm. 6) yields

$$
\left|B^{0}\left(v^{H} ; w^{H}\right)-\hat{B}^{0}\left(v^{H} ; w^{H}\right)\right| \leq C H^{2}\left\|\mathcal{A}^{0}\left(x, \nabla v^{H}\right)\right\|_{\bar{H}^{2}(\Omega)}\left\|\nabla w^{H}\right\|_{L^{2}(\Omega)},
$$

where $\|\cdot\|_{H^{2}(\Omega)}^{2}=\sum_{K \in \mathcal{T}_{H}}\|\cdot\|_{H^{2}(K)}^{2}$ denotes a broken Sobolev norm. Let the $k$ th coordinate function of $\mathcal{A}^{0}$ be denoted by $\mathcal{A}_{(k)}^{0}$, for $1 \leq k \leq d$. Then, for $1 \leq i, j, k \leq d$ and a.e. $x \in \Omega$, the (weak) derivatives of $\mathcal{A}^{0}\left(x, \nabla v^{H}\right)$ are given by

$$
\partial_{x_{i}}\left[\mathcal{A}_{(k)}^{0}\left(x, \nabla v^{H}(x)\right)\right]=\partial_{x_{i}} \mathcal{A}_{(k)}^{0}\left(x, \nabla v^{H}(x)\right), \partial_{x_{j} x_{i}}\left[\mathcal{A}_{(k)}^{0}\left(x, \nabla v^{H}(x)\right)\right]=\partial_{x_{j} x_{i}} \mathcal{A}_{(k)}^{0}\left(x, \nabla v^{H}(x)\right),
$$

as $\nabla v^{H}$ is piecewise constant. We conclude the proof by observing that for any $K \in \mathcal{T}_{H}$ we have $\left\|\mathcal{A}^{0}\left(x, \nabla v^{H}\left(x_{K}\right)\right)\right\|_{H^{2}(K)} \leq C\left(L_{0}+\left|\nabla v^{H}\left(x_{K}\right)\right|\right) \sqrt{|K|}$ due to (4.4b).

Remark 5.19. For a linear problem $\mathcal{A}^{0}(x, \xi)=a^{0}(x) \xi$, with $a^{0} \in\left(L^{\infty}(\Omega)\right)^{d \times d}$, the regularity assumption of (4.4b) with $\mu=2$ becomes $a^{0} \in W^{2, \infty}(\Omega)$, which is used for FEM based on numerical integration for linear problems, see [43]. Then, the bounds of $(4.4 \mathrm{~b})$ are valid for $L_{0}=0$.

With the Lemma 5.18 at hand, the term (5.1f) can be estimated immediately.

Corollary 5.20. Let $p=2$, assume that $\mathcal{A}^{0}$ satisfies $\left(\mathcal{A}_{0-2}\right)$ with $\alpha=1, \beta=2$ and $\mathcal{A}^{0}(x, \cdot) \in \mathcal{C}^{1}\left(\mathbb{R}^{d} ; \mathbb{R}^{d}\right)$ for a.e. $x \in \Omega$. Let $u^{0}$ be the solution of the homogenized problem (2.3), $\tilde{u}^{H, 0}$ its elliptic projection (5.19) and consider the maps $B^{0}$ and $\hat{B}^{0}$ given by (3.10) and (3.11). If $\mathcal{A}^{0}$ satisfies the hypothesis (4.4b) for $\mu=2$ and $u^{0} \in \mathcal{C}^{0}\left([0, T], H^{1}(\Omega)\right)$, then we have for $\mathcal{U}_{n+1}^{H}=\tilde{u}_{n+1}^{H, 0}$

$$
\left|B^{0}\left(\mathcal{U}_{n+1}^{H} ; w^{H}\right)-\hat{B}^{0}\left(\mathcal{U}_{n+1}^{H} ; w^{H}\right)\right| \leq C H^{2}\left(L_{0}+\left\|u^{0}\right\|_{\mathcal{C}^{0}\left([0, T], H^{1}(\Omega)\right)}\right)\left\|\nabla w^{H}\right\|_{L^{2}(\Omega)},
$$

for $0 \leq n \leq N-1$ and every $w^{H} \in S_{0}^{1}\left(\Omega, \mathcal{T}_{H}\right)$ with a constant $C$ independent of $\Delta t$ and $H$. 
Proof of $\mathcal{C}^{0}\left(L^{2}\right)$ estimate from Theorem 4.4. Recall that $\theta_{n}^{H}=u_{n}^{H}-\mathcal{U}_{n}^{H}$ for $0 \leq n \leq N$. For $\mu=2$, we set $\mathcal{U}_{n}^{H}=\tilde{u}_{n}^{H, 0}$. By estimating the terms (5.1d), (5.1e) and (5.1f) using Lemmas 5.16, 5.17 and Corollary 5.20 (instead of Lems. 5.5, 5.7, 5.8), we obtain the estimate (5.16) with a term of order $H^{2}$ instead of $H$. Then the result is obtained by combining that with Lemma 5.14 .

\subsubsection{Explicit estimates for the HMM upscaling error}

In this section, for $p=2$ and $\alpha=1, \beta=2$ in $\left(\mathcal{A}_{1-2}\right)$, we prove explicit estimates for the upscaling error decomposed into micro and modeling error, which are studied in separate paragraphs.

Micro error. In a first part, we bound the micro error $r_{\text {mic }}$ defined in (4.2a) which is due to the finite element approximation of the micro problems (3.6). Under classical regularity assumptions on $\bar{\chi}_{K}^{\xi}$ solving (3.8), see (H1), we derive a robust linear convergence rate, see Lemma 5.21. Introducing an auxiliary adjoint problem (5.25) and assuming regularity $\left(\mathbf{H} \mathbf{1}^{*}\right)$ of its solution, a robust quadratic convergence can be shown in Lemma 5.23.

We start by deriving a first estimate for the micro error $r_{\text {mic }}$.

Lemma 5.21. Let $p=2$, assume that $\mathcal{A}^{\varepsilon}$ satisfies $\left(\mathcal{A}_{0-2}\right)$ with $\alpha=1, \beta=2$ and that $(\mathbf{H 1})$ holds. For any $v^{H} \in S_{0}^{1}\left(\Omega, \mathcal{T}_{H}\right)$ and either periodic coupling $W\left(K_{\delta}\right)=\mathcal{W}_{\text {per }}^{1,2}\left(K_{\delta}\right)$ or Dirichlet coupling $W\left(K_{\delta}\right)=H_{0}^{1}\left(K_{\delta}\right)$ for the micro problems (3.6), the micro error $r_{\text {mic }}\left(\nabla v^{H}\right)$, see (4.2a), can be estimated by

$$
r_{\text {mic }}\left(\nabla v^{H}\right) \leq C \frac{h}{\varepsilon}\left(L_{0}+\left\|\nabla v^{H}\right\|_{L^{2}(\Omega)}\right),
$$

where $C$ is independent of $H, h, \delta$ and $\varepsilon$.

Proof. Let $\xi \in \mathbb{R}^{d}, K \in \mathcal{T}_{H}$ and $\bar{\chi}_{K}^{\xi} \in W\left(K_{\delta}\right)$ and $\chi_{K}^{\xi, h} \in S^{1}\left(K_{\delta}, \mathcal{T}_{h}\right)$ be the solution of (3.8) and (3.7), respectively, with the same coupling condition. Observing that $\beta-\alpha=1$, choosing $z^{h}=\mathcal{I}_{h} \bar{\chi}_{K}^{\xi}$ (the nodal interpolant on $K_{\delta}$ ) and using the interpolation estimate (5.4a) and (H1) we get from (5.7) that

$$
\left\|\nabla \bar{\chi}_{K}^{\xi}-\nabla \chi_{K}^{\xi, h}\right\|_{L^{2}\left(K_{\delta}\right)} \leq C \frac{h}{\varepsilon}\left(L_{0}+|\xi|\right) \sqrt{\left|K_{\delta}\right|} .
$$

Choosing then $z^{h}=\chi_{K}^{\xi, h}$ with $\xi=\nabla v^{H}\left(x_{K}\right)$ in Lemma 5.9 on every $K \in \mathcal{T}_{H}$ and observing that $\gamma=\alpha /(\beta-\alpha)=$ 1 , we conclude the proof by applying (5.24).

In $[1,2,9]$ a convergence of the order $(h / \varepsilon)^{2}$ has been shown for linear micro problems (3.6), i.e., for data $\mathcal{A}^{\varepsilon}(x, \xi)=a^{\varepsilon}(x) \xi$. Thus, the estimate of Lemma 5.21 is in general non-optimal. We note that an adjoint micro problem was used to prove the quadratic convergence for non-symmetric tensors $a^{\varepsilon}(x)$ (see [9], Lem. 4.6) for a short proof. In this view, we introduce the following linear auxiliary micro problems: for $\xi \in \mathbb{R}^{d}, 1 \leq j \leq d$ and $K \in \mathcal{T}_{H}$, find $\bar{X}_{K}^{\xi, j} \in W\left(K_{\delta}\right)$ such that

$$
\int_{K_{\delta}}\left(D_{\xi} \mathcal{A}^{\varepsilon}\left(x, \xi+\nabla \bar{\chi}_{K}^{\xi}\right)\right)^{T}\left(e_{j}+\nabla \bar{X}_{K}^{\xi, j}\right) \cdot \nabla z \mathrm{~d} x=0, \quad \forall z \in W\left(K_{\delta}\right),
$$

where $K_{\delta}$ is the sampling domain associated to $K$ and $\bar{\chi}_{K}^{\xi}$ solves the cell problem (3.8). We note that problem (5.25) admits a unique solution if $\mathcal{A}^{\varepsilon}$ satisfies $\left(\mathcal{A}_{0-2}\right)$ and $\mathcal{A}^{\varepsilon}(x, \cdot) \in \mathcal{C}^{1}\left(\mathbb{R}^{d} ; \mathbb{R}^{d}\right)$, as then the Jacobian $D_{\xi} \mathcal{A}^{\varepsilon}$ is uniformly bounded and elliptic, see Remark 5.11.

Remark 5.22. We note, that for a linear map $\mathcal{A}^{\varepsilon}(x, \xi)=a^{\varepsilon}(x) \xi$ the derivative $D_{\xi} \mathcal{A}^{\varepsilon}$ is simply given by $D_{\xi} \mathcal{A}^{\varepsilon}(x, \xi)=a^{\varepsilon}(x)$. Thus, the auxiliary micro problem (5.25) reduces to

$$
\int_{K_{\delta}} a^{\varepsilon}(x)^{T}\left(e_{j}+\nabla \bar{X}_{K}^{j}\right) \cdot \nabla z \mathrm{~d} x=0, \quad \forall z \in W\left(K_{\delta}\right),
$$

which is independent of $\xi$ and the corrector $\bar{\chi}_{K}^{\xi}$. Indeed, we recover the adjoint micro problem used to analyze linear homogenization problems, e.g., see [9]. 
Lemma 5.23. Let $p=2$, assume that $\mathcal{A}^{\varepsilon}$ satisfies $\left(\mathcal{A}_{0-2}\right)$ with $\alpha=1, \beta=2, \mathcal{A}^{\varepsilon}(x, \cdot) \in W^{2, \infty}\left(\mathbb{R}^{d} ; \mathbb{R}^{d}\right)$ and (H1), (H1*) hold. For any $v^{H} \in S_{0}^{1}\left(\Omega, \mathcal{T}_{H}\right)$ and either periodic coupling $W\left(K_{\delta}\right)=\mathcal{W}_{\text {per }}^{1,2}\left(K_{\delta}\right)$ or Dirichlet coupling $W\left(K_{\delta}\right)=H_{0}^{1}\left(K_{\delta}\right)$ for the micro problems (3.6), the micro error $r_{\text {mic }}\left(\nabla v^{H}\right)$, see (4.2a), can be estimated by

$$
r_{\text {mic }}\left(\nabla v^{H}\right) \leq C\left(\frac{h}{\varepsilon}\right)^{2}\left(L_{0}+L_{0}^{2}+\left\|\nabla v^{H}\right\|_{L^{2}(\Omega)}+\left\|\nabla v^{H}\right\|_{L^{4}(\Omega)}^{2}\right),
$$

where $C$ is independent of $H, h, \delta$ and $\varepsilon$.

Proof. Like in Lemma 5.9, we estimate the difference $\mathcal{A}_{K}^{0, h}(\xi)-\overline{\mathcal{A}}_{K}^{0}(\xi)$ for $\xi \in \mathbb{R}^{d}$ and $K \in \mathcal{T}_{H}$ (with associated sampling domain $K_{\delta}$ ), where $\overline{\mathcal{A}}_{K}^{0}(\xi)$ and $\mathcal{A}_{K}^{0, h}(\xi)$ are given by (3.9). They are based on the solutions $\bar{\chi}_{K}^{\xi}$ and $\chi_{K}^{\xi, h}$ to the micro problems (3.8) and (3.7), respectively, solved with the same coupling condition. Let $1 \leq j \leq d$, then

$$
\begin{aligned}
\overline{\mathcal{A}}_{K}^{0}(\xi) \cdot e_{j}-\mathcal{A}_{K}^{0, h}(\xi) \cdot e_{j} & =\frac{1}{\left|K_{\delta}\right|} \int_{K_{\delta}}\left[\mathcal{A}^{\varepsilon}\left(x, \xi+\nabla \bar{\chi}_{K}^{\xi}\right)-\mathcal{A}^{\varepsilon}\left(x, \xi+\nabla \chi_{K}^{\xi, h}\right)\right] \cdot e_{j} \mathrm{~d} x \\
& =\frac{1}{\left|K_{\delta}\right|} \int_{K_{\delta}}\left[\mathcal{A}^{\varepsilon}\left(x, \xi+\nabla \bar{\chi}_{K}^{\xi}\right)-\mathcal{A}^{\varepsilon}\left(x, \xi+\nabla \chi_{K}^{\xi, h}\right)\right] \cdot\left(e_{j}+\nabla \mathcal{I}_{h} \bar{X}_{K}^{\xi, j}\right) \mathrm{d} x,
\end{aligned}
$$

where the Galerkin orthogonality for monotone FEM is used and $\mathcal{I}_{h} \bar{X}_{K}^{\xi, j} \in S^{1}\left(K_{\delta}, \mathcal{T}_{h}\right)$ is the nodal interpolant of $\bar{X}_{K}^{\xi, j}$ on $K_{\delta}$. Further, we apply the Taylor formula (5.17) and use that $\bar{X}_{K}^{\xi, j}$ solves $(5.25)$

$$
\begin{aligned}
{\left[\mathcal{A}_{K}^{0, h}(\xi)-\overline{\mathcal{A}}_{K}^{0}(\xi)\right] \cdot e_{j}=} & \frac{1}{\left|K_{\delta}\right|} \int_{K_{\delta}} D_{\xi} \mathcal{A}^{\varepsilon}\left(x, \xi+\nabla \bar{\chi}_{K}^{\xi}\right)\left(\nabla \chi_{K}^{\xi, h}-\nabla \bar{\chi}_{K}^{\xi}\right) \cdot\left(e_{j}+\nabla \mathcal{I}_{h} \bar{X}_{K}^{\xi, j}\right) \mathrm{d} x \\
& +\frac{1}{\left|K_{\delta}\right|} \int_{K_{\delta}} \int_{0}^{1} D_{\xi} \mathcal{A}^{\varepsilon}\left(x, \xi+\nabla \bar{\chi}_{K}^{\xi}+\tau\left(\nabla \chi_{K}^{\xi, h}-\nabla \bar{\chi}_{K}^{\xi}\right)\right)-D_{\xi} \mathcal{A}^{\varepsilon}\left(x, \xi+\nabla \bar{\chi}_{K}^{\xi}\right) \mathrm{d} \tau \\
& \times\left(\nabla \chi_{K}^{\xi, h}-\nabla \bar{\chi}_{K}^{\xi}\right) \cdot\left(e_{j}+\nabla \mathcal{I}_{h} \bar{X}_{K}^{\xi, j}\right) \mathrm{d} x \\
= & \frac{1}{\left|K_{\delta}\right|} \int_{K_{\delta}} D_{\xi} \mathcal{A}^{\varepsilon}\left(x, \xi+\nabla \bar{\chi}_{K}^{\xi}\right)\left(\nabla \chi_{K}^{\xi, h}-\nabla \bar{\chi}_{K}^{\xi}\right) \cdot\left(\nabla \mathcal{I}_{h} \bar{X}_{K}^{\xi, j}-\nabla \bar{X}_{K}^{\xi, j}\right) \mathrm{d} x \\
& +\frac{1}{\left|K_{\delta}\right|} \int_{K_{\delta}} \int_{0}^{1} D_{\xi} \mathcal{A}^{\varepsilon}\left(x, \xi+\nabla \bar{\chi}_{K}^{\xi}+\tau\left(\nabla \chi_{K}^{\xi, h}-\nabla \bar{\chi}_{K}^{\xi}\right)\right)-D_{\xi} \mathcal{A}^{\varepsilon}\left(x, \xi+\nabla \bar{\chi}_{K}^{\xi}\right) \mathrm{d} \tau \\
& \times\left(\nabla \chi_{K}^{\xi, h}-\nabla \bar{\chi}_{K}^{\xi}\right) \cdot\left(e_{j}+\nabla \mathcal{I}_{h} \bar{X}_{K}^{\xi, j}\right) \mathrm{d} x .
\end{aligned}
$$

Then, the uniform boundedness and the Lipschitz continuity of $D_{\xi} \mathcal{A}^{\varepsilon}(x, \cdot)$ yield

$$
\begin{aligned}
\left|\overline{\mathcal{A}}_{K}^{0}(\xi) \cdot e_{j}-\mathcal{A}_{K}^{0, h}(\xi) \cdot e_{j}\right| \leq & \frac{L}{\left|K_{\delta}\right|}\left\|\nabla \chi_{K}^{\xi, h}-\nabla \bar{\chi}_{K}^{\xi}\right\|_{L^{2}\left(K_{\delta}\right)}\left\|\nabla \mathcal{I}_{h} \bar{X}_{K}^{\xi, j}-\nabla \bar{X}_{K}^{\xi, j}\right\|_{L^{2}\left(K_{\delta}\right)} \\
& +\frac{C}{\left|K_{\delta}\right|}\left\|\nabla \chi_{K}^{\xi, h}-\nabla \bar{\chi}_{K}^{\xi}\right\|_{L^{2}\left(K_{\delta}\right)}^{2}\left(1+\left|\mathcal{I}_{h} \bar{X}_{K}^{\xi, j}\right|_{W^{1, \infty}\left(K_{\delta}\right)}\right) \\
\leq & C\left(\frac{h}{\varepsilon}\right)^{2}\left(L_{0}+L_{0}^{2}+|\xi|+|\xi|^{2}\right)\left(1+\left|\mathcal{I}_{h} \bar{X}_{K}^{\xi, j}\right|_{W^{1, \infty}\left(K_{\delta}\right)}\right),
\end{aligned}
$$

where we applied estimate (5.24) (using assumption (H1)) and the standard $H^{1}$ interpolation error estimate (5.4a) for the nodal interpolation operator $\mathcal{I}_{h}$ on $K_{\delta}$ (using assumption (i) from $\left(\mathbf{H} 1^{*}\right)$ ). Further, the bound (5.4b) for $\mathcal{I}_{h}$ and hypothesis (ii) from $\left(\mathbf{H 1}^{*}\right)$ yield $\left|\mathcal{I}_{h} \bar{X}_{K}^{\xi, j}\right|_{W^{1, \infty}\left(K_{\delta}\right)} \leq C$. Then, the result follows from the definition (4.2a) of $r_{\text {mic }}$. 
Proof of Theorem 4.5. We combine the results of Theorem 4.4 with the estimates of Lemmas 5.21 and 5.23, with $v^{H}=\mathcal{U}_{n}^{H}$ for $1 \leq n \leq N$, for linear and quadratic micro convergence, respectively. We note that $\left\|\nabla \mathcal{U}_{n}^{H}\right\|_{L^{2}(\Omega)}$ and $\left\|\nabla \mathcal{U}_{n}^{H}\right\|_{L^{4}(\Omega)}$ are bounded for both $\mathcal{U}_{n}^{H}=\mathcal{I}_{H} u^{0}\left(\cdot, t_{n}\right)$ the nodal interpolant of the homogenized solution $u^{0}$ and $\mathcal{U}_{n}^{H}=\tilde{u}_{n}^{H, 0}$ the elliptic projection (5.19). In particular, we have $\left\|\nabla \mathcal{I}_{H} u^{0}\left(\cdot, t_{n}\right)\right\|_{L^{4}(\Omega)} \leq C\left\|u^{0}\left(\cdot, t_{n}\right)\right\|_{H^{2}(\Omega)}$, from classical interpolation results (see [16], Thm. 3.1.6), and Lemma 5.15 yields $\left\|\nabla \tilde{u}_{n}^{H, 0}\right\|_{L^{4}(\Omega)} \leq C\left\|u^{0}\left(\cdot, t_{n}\right)\right\|_{W^{1, \infty}(\Omega)}$.

Modeling error. In the second part, for locally periodic maps $\mathcal{A}^{\varepsilon}$, we prove explicit bounds for the modeling error $r_{\text {mod }}$ defined in (4.2b) including the influence of the boundary conditions chosen for $W\left(K_{\delta}\right)$ in $(3.5)$, the sampling domain size $\delta$ and the absence of collocation of $\mathcal{A}(x, x / \varepsilon, \xi)$ in the slow variable $x$.

Periodic homogenization. As we are considering locally periodic maps $\mathcal{A}^{\varepsilon}$ independent of the time variable $t$, we can use the representation of $\mathcal{A}^{0}$ derived in the case of monotone elliptic problems $-\operatorname{div}\left(\mathcal{A}^{\varepsilon}\left(x, \nabla u^{\varepsilon}\right)\right)=f$ in $\Omega$ (see [44], Thm. 8.1). Assume that $\mathcal{A}^{\varepsilon}(x, \xi)=\mathcal{A}(x, x / \varepsilon, \xi)$ where $\mathcal{A}(x, y, \xi)$ is $Y$-periodic in $y$, i.e., $\mathcal{A}^{\varepsilon}$ is locally periodic. Then (see [33], Sect. 3), the homogenized map $\mathcal{A}^{0}$ is explicitly given by

$$
\mathcal{A}^{0}(x, \xi)=\int_{Y} \mathcal{A}\left(x, y, \xi+\nabla \chi^{\xi}(x, y)\right) \mathrm{d} y,
$$

where $x \in \Omega, \xi \in \mathbb{R}^{d}$ and $\chi^{\xi}(x, \cdot) \in \mathcal{W}_{\text {per }}^{1,2}(Y)$ solves the cell problem: find $\chi^{\xi}(x, \cdot) \in \mathcal{W}_{\text {per }}^{1,2}(Y)$ such that

$$
\int_{Y} \mathcal{A}\left(x, y, \xi+\nabla \chi^{\xi}(x, y)\right) \cdot \nabla z \mathrm{~d} y=0, \quad \forall z \in \mathcal{W}_{\text {per }}^{1,2}(Y)
$$

Collocation in the slow variable. If the decomposition $\mathcal{A}(x, x / \varepsilon, \xi)$ between macro and micro scale is explicitly known, we can collocate the map $\mathcal{A}^{\varepsilon}$ in the slow variable $x$ at the quadrature nodes $x_{K}$. Then, for $\xi \in \mathbb{R}^{d}$ and $K \in \mathcal{T}_{H}$, the collocated micro problem reads as: find $\tilde{\chi}_{K}^{\xi} \in W\left(K_{\delta}\right)$ such that

$$
\int_{K_{\delta}} \mathcal{A}\left(x_{K}, \frac{x}{\varepsilon}, \xi+\nabla \tilde{\chi}_{K}^{\xi}\right) \cdot \nabla z \mathrm{~d} x=0, \quad \forall z \in W\left(K_{\delta}\right)
$$

and, analogously to (3.9), the homogenized map $\tilde{\mathcal{A}}_{K}^{0}$ can be defined

$$
\tilde{\mathcal{A}}_{K}^{0}(\xi)=\frac{1}{\left|K_{\delta}\right|} \int_{K_{\delta}} \mathcal{A}\left(x_{K}, \frac{x}{\varepsilon}, \xi+\nabla \tilde{\chi}_{K}^{\xi}\right) \mathrm{d} x .
$$

If a locally periodic map $\mathcal{A}^{\varepsilon}$ is not collocated in the slow variable, a modeling error of order $\mathcal{O}(\delta)$ is introduced. In particular, if $\mathcal{A}^{\varepsilon}(x)$ satisfies $(\mathbf{H 2})$, one can show that

$$
\left|\tilde{\mathcal{A}}_{K}^{0}(\xi)-\overline{\mathcal{A}}_{K}^{0}(\xi)\right| \leq C \delta\left(L_{0}+|\xi|\right),
$$

where the homogenized maps $\overline{\mathcal{A}}_{K}^{0}$ and $\tilde{\mathcal{A}}_{K}^{0}$ are given in (3.9) and (5.30), respectively. To obtain estimate (5.31), we first prove (similar to the upper bound of Lem. 3.4) the bound $\left\|\nabla \tilde{\chi}_{K}^{\xi}\right\|_{L^{2}\left(K_{\delta}\right)}+\left\|\nabla \bar{\chi}_{K}^{\xi}\right\|_{L^{2}\left(K_{\delta}\right)} \leq C\left(L_{0}+\right.$ $|\xi|) \sqrt{\left|K_{\delta}\right|}$ for the solution $\tilde{\chi}_{K}^{\xi}$ and $\bar{\chi}_{K}^{\xi}$ to (5.29) and (3.8), respectively, and we then show (using the Lipschitz continuity assumed in (H2)) that $\left\|\nabla \tilde{\chi}_{K}^{\xi}-\nabla \bar{\chi}_{K}^{\xi}\right\|_{L^{2}\left(K_{\delta}\right)} \leq C \delta\left(L_{0}+|\xi|\right) \sqrt{\left|K_{\delta}\right|}$.

Periodic boundary conditions. We next show that periodic coupling with a sampling domain size $\delta$ taken as an integer multiple of $\varepsilon$ is optimal for locally periodic data.

Let $\xi \in \mathbb{R}^{d}$ and $K \in \mathcal{T}_{H}$ and let $\chi^{\xi}\left(x_{K}, \cdot\right)$ and $\tilde{\chi}_{K}^{\xi}$ be the solution to (5.28) and (5.29), respectively. We observe that $\chi^{\xi}\left(x_{K}, x / \varepsilon\right)=\tilde{\chi}_{K}^{\xi}(x)$ on $K_{\delta}$ (if $\delta>\varepsilon, \chi^{\xi}\left(x_{K}, \cdot\right)$ is periodically extended) and thus

$$
\mathcal{A}^{0}\left(x_{K}, \xi\right)=\tilde{\mathcal{A}}_{K}^{0}(\xi), \quad \xi \in \mathbb{R}^{d}, K \in \mathcal{T}_{H},
$$

for the maps $\mathcal{A}^{0}\left(x_{K}, \cdot\right)$ and $\tilde{\mathcal{A}}_{K}^{0}$ defined in (5.27) and (5.30), respectively.

Dirichlet boundary conditions. In contrast to the optimal periodic coupling with $\delta / \varepsilon \in \mathbb{N}$, using Dirichlet coupling with $\delta \geq \varepsilon$ leads to resonance errors due to the artificial boundary conditions. 
Lemma 5.24. Let $p=2$ and assume that $\mathcal{A}^{\varepsilon}$ satisfies $\left(\mathcal{A}_{0-2}\right)$ with $\alpha=1, \beta=2$ and $(\mathbf{H 2})$. Let $\xi \in \mathbb{R}^{d}$, $K \in \mathcal{T}_{H}$ and the maps $\tilde{\mathcal{A}}_{K}^{0}$ and $\mathcal{A}^{0}\left(x_{K}, \cdot\right)$ be given by (5.30) and (5.27), respectively. Further, assume that the exact corrector $\chi^{\xi}\left(x_{K}, \cdot\right)$ solving the cell problem (5.28) satisfies $\chi^{\xi}\left(x_{K}, \cdot\right) \in W^{1, \infty}(Y)$. Then, for Dirichlet coupling $W\left(K_{\delta}\right)=H_{0}^{1}\left(K_{\delta}\right)$ and a sampling domain size $\delta>\varepsilon$ it holds

$$
\left|\mathcal{A}^{0}\left(x_{K}, \xi\right)-\tilde{\mathcal{A}}_{K}^{0}(\xi)\right| \leq C\left(\frac{\varepsilon}{\delta}\right)^{1 / 2}\left(L_{0}+|\xi|+\left\|\chi^{\xi}\left(x_{K}, y\right)\right\|_{W^{1, \infty}(Y)}\right),
$$

where $C$ is independent of $\xi, \delta$ and $\varepsilon$.

Proof. We use the techniques used to analyze the resonance error for linear homogenization problems (see [24], Thm. 1.2). Let $n \in \mathbb{N}$ be given by $n=\lfloor\delta / \varepsilon\rfloor($ if $\delta / \varepsilon \notin \mathbb{N}$ ), or $n=\delta / \varepsilon-1$ (if $\delta / \varepsilon \in \mathbb{N} \backslash\{0\}$ ). Further, we define $K_{\Gamma}=K_{\delta} \backslash K_{n \varepsilon}$ and we observe that $\left|K_{\Gamma}\right| \leq C \varepsilon \delta^{d-1}$. Then we decompose the difference $\tilde{\mathcal{A}}_{K}^{0}-\mathcal{A}^{0}\left(x_{K}, \xi\right)$ into two terms according to

$$
\begin{aligned}
\tilde{\mathcal{A}}_{K}^{0}(\xi) & -\mathcal{A}^{0}\left(x_{K}, \xi\right)=\frac{1}{\left|K_{\delta}\right|} \int_{K_{\delta}} \mathcal{A}\left(x_{K}, \frac{x}{\varepsilon}, \xi+\nabla \tilde{\chi}_{K}^{\xi}\right)-\mathcal{A}\left(x_{K}, \frac{x}{\varepsilon}, \xi+\nabla \chi^{\xi}\left(x_{K}, \frac{x}{\varepsilon}\right)\right) \mathrm{d} x \\
& +\frac{1}{\left|K_{\delta}\right|} \int_{K_{\delta}} \mathcal{A}\left(x_{K}, \frac{x}{\varepsilon}, \xi+\nabla \chi^{\xi}\left(x_{K}, \frac{x}{\varepsilon}\right)\right) \mathrm{d} x-\frac{1}{\left|K_{n \varepsilon}\right|} \int_{K_{n \varepsilon}} \mathcal{A}\left(x_{K}, \frac{x}{\varepsilon}, \xi+\nabla \chi^{\xi}\left(x_{K}, \frac{x}{\varepsilon}\right)\right) \mathrm{d} x
\end{aligned}
$$

where $\chi^{\xi}\left(x_{K}, y\right)$ is extended periodically to $\mathbb{R}^{d}$ and the first and second line is denoted by $I_{1}$ and $I_{2}$, respectively. First, we estimate $I_{2}$ similarly as for the linear case

$$
\begin{aligned}
I_{2} & =\frac{1}{\left|K_{\delta}\right|} \int_{K_{\Gamma}} \mathcal{A}\left(x_{K}, \frac{x}{\varepsilon}, \xi+\nabla \chi^{\xi}\left(x_{K}, \frac{x}{\varepsilon}\right)\right) \mathrm{d} x+\left(\frac{1}{\left|K_{\delta}\right|}-\frac{1}{\left|K_{n \varepsilon}\right|}\right) \int_{K_{n \varepsilon}} \mathcal{A}\left(x_{K}, \frac{x}{\varepsilon}, \xi+\nabla \chi^{\xi}\left(x_{K}, \frac{x}{\varepsilon}\right)\right) \mathrm{d} x \\
& \leq C\left(\frac{\left|K_{\Gamma}\right|}{\left|K_{\delta}\right|}+\frac{\left|K_{\Gamma}\right|}{\left|K_{\delta}\right|\left|K_{n \varepsilon}\right|}\left|K_{n \varepsilon}\right|\right)\left(L_{0}+|\xi|+\left|\chi^{\xi}\left(x_{K}, y\right)\right|_{W^{1, \infty}(Y)}\right) \\
& \leq C \frac{\varepsilon}{\delta}\left(L_{0}+|\xi|+\left|\chi^{\xi}\left(x_{K}, y\right)\right|_{W^{1, \infty}(Y)}\right),
\end{aligned}
$$

using the estimate (3.15) for $\mathcal{A}$ and the assumption $\chi^{\xi}\left(x_{K}, \cdot\right) \in W^{1, \infty}(Y)$. To estimate the term $I_{1}$ we define the function $\theta^{\xi}(x)=\tilde{\chi}_{K}^{\xi}(x)-\varepsilon \chi^{\xi}\left(x_{K}, x / \varepsilon\right)$ on $K_{\delta}$ (using the periodic extension of $\chi^{\xi}\left(x_{K}, \cdot\right)$ ). As $\left.\tilde{\chi}_{K}^{\xi}\right|_{\partial K_{\delta}}=0$ (in the sense of traces), we decompose $\theta^{\xi}$ into

$$
\theta^{\xi}(x)=\theta_{0}^{\xi}(x)-\varepsilon \chi^{\xi}\left(x_{K}, \frac{x}{\varepsilon}\right)\left(1-\rho_{\varepsilon}(x)\right), \quad x \in K_{\delta},
$$

where $\theta_{0}^{\xi} \in H_{0}^{1}\left(K_{\delta}\right)$ and $\rho_{\varepsilon}: K_{\delta} \rightarrow \mathbb{R}$ is a smooth cut-off function satisfying $\rho_{\varepsilon} \equiv 1$ in $K_{n \varepsilon},\left.\rho_{\varepsilon}\right|_{\partial K_{\delta}} \equiv 0$ and $\left|\nabla \rho_{\varepsilon}\right| \leq C \varepsilon^{-1}$ in $K_{\Gamma}$ (where $C$ is independent of $\delta$ and $\varepsilon$ ). Using the strong monotonicity $\left(\mathcal{A}_{2}\right)$ of $\mathcal{A}$ and the decomposition (5.34) of $\theta^{\xi}$ we obtain

$$
\begin{aligned}
& \lambda\left\|\nabla \tilde{\chi}_{K}^{\xi}(x)-\nabla \chi^{\xi}\left(x_{K}, \frac{x}{\varepsilon}\right)\right\|_{L^{2}\left(K_{\delta}\right)}^{2} \\
& \leq \int_{K_{\delta}}\left[\mathcal{A}\left(x_{K}, \frac{x}{\varepsilon}, \xi+\nabla \tilde{\chi}_{K}^{\xi}(x)\right)-\mathcal{A}\left(x_{K}, \frac{x}{\varepsilon}, \xi+\nabla \chi^{\xi}\left(x_{K}, \frac{x}{\varepsilon}\right)\right)\right] \cdot\left(\nabla \tilde{\chi}_{K}^{\xi}(x)-\nabla \chi^{\xi}\left(x_{K}, \frac{x}{\varepsilon}\right)\right) \mathrm{d} x \\
&=\int_{K_{\delta}} \mathcal{A}\left(x_{K}, \frac{x}{\varepsilon}, \xi+\nabla \tilde{\chi}_{K}^{\xi}(x)\right) \cdot \nabla \theta_{0}^{\xi} \mathrm{d} x-\int_{K_{\delta}} \mathcal{A}\left(x_{K}, \frac{x}{\varepsilon}, \xi+\nabla \chi^{\xi}\left(x_{K}, \frac{x}{\varepsilon}\right)\right) \cdot \nabla \theta_{0}^{\xi} \mathrm{d} x \\
& \quad+\int_{K_{\delta}}\left[\mathcal{A}\left(x_{K}, \frac{x}{\varepsilon}, \xi+\nabla \tilde{\chi}_{K}^{\xi}(x)\right)-\mathcal{A}\left(x_{K}, \frac{x}{\varepsilon}, \xi+\nabla \chi^{\xi}\left(x_{K}, \frac{x}{\varepsilon}\right)\right)\right] \cdot \nabla\left[\varepsilon \chi^{\xi}\left(x_{K}, \frac{x}{\varepsilon}\right)\left(1-\rho_{\varepsilon}(x)\right)\right] \mathrm{d} x \\
&=: J_{1}-J_{2}+J_{3} .
\end{aligned}
$$


First, as $\tilde{\chi}_{K}^{\xi}$ solves the cell problem (5.29) in the space $W\left(K_{\delta}\right)=H_{0}^{1}\left(K_{\delta}\right)$ we have $J_{1}=0$. To show that the second term $J_{2}$ vanishes as well we define $\theta_{\text {per }}^{\xi} \in \mathcal{W}_{\text {per }}^{1,2}\left(K_{(n+1) \varepsilon}\right)$ by

$$
\theta_{\mathrm{per}}^{\xi}(x)=\theta_{0}^{\xi}(x)-c_{\theta}^{\xi}, \quad\left(\text { if } x \in K_{\delta}\right), \quad \theta_{\mathrm{per}}^{\xi}(x)=-c_{\theta}^{\xi}, \quad\left(\text { if } x \in K_{(n+1) \varepsilon} \backslash K_{\delta}\right),
$$

with $c_{\theta}^{\xi}=\frac{1}{\left|K_{(n+1) \varepsilon}\right|} \int_{K_{\delta}} \theta_{0}^{\xi}(x) \mathrm{d} x$. Thus, we observe that $\nabla \theta_{\mathrm{per}}^{\xi}=\nabla \theta^{\xi}$ on $K_{\delta}$ and $\nabla \theta_{\mathrm{per}}^{\xi}=0$ on $K_{(n+1) \varepsilon} \backslash K_{\delta}$. Hence, when scaling and periodically extending (5.28) we get

$$
J_{2}=\int_{K_{(n+1) \varepsilon}} \mathcal{A}\left(x_{K}, \frac{x}{\varepsilon}, \xi+\nabla \chi^{\xi}\left(x_{K}, \frac{x}{\varepsilon}\right)\right) \cdot \nabla \theta_{\mathrm{per}}^{\xi}(x) \mathrm{d} x=0 .
$$

Further, using the Lipschitz continuity $\left(\mathcal{A}_{1}\right)$ of $\mathcal{A}$, we estimate the term $J_{3}$ as

$$
\begin{aligned}
\left|J_{3}\right| & \leq L\left\|\nabla \tilde{\chi}_{K}^{\xi}(x)-\nabla \chi^{\xi}\left(x_{K}, \frac{x}{\varepsilon}\right)\right\|_{L^{2}\left(K_{\delta}\right)}\left\|\nabla \chi^{\xi}\left(x_{K}, \frac{x}{\varepsilon}\right)\left(1-\rho_{\varepsilon}(x)\right)-\varepsilon \nabla \rho_{\varepsilon}(x) \chi^{\xi}\left(x_{K}, \frac{x}{\varepsilon}\right)\right\|_{L^{2}\left(K_{\Gamma}\right)} \\
& \leq C \sqrt{\left|K_{\Gamma}\right|}\left\|\nabla \tilde{\chi}_{K}^{\xi}(x)-\nabla \chi^{\xi}\left(x_{K}, \frac{x}{\varepsilon}\right)\right\|_{L^{2}\left(K_{\delta}\right)}\left\|\chi^{\xi}\left(x_{K}, y\right)\right\|_{W^{1, \infty}(Y)},
\end{aligned}
$$

where we used the properties of $\rho_{\varepsilon}$, in particular, $1-\rho_{\varepsilon}(x) \equiv 0$ on $K_{n \varepsilon}$ and $\nabla \rho_{\varepsilon} \leq C \varepsilon^{-1}$. Combining that $J_{1}=J_{2}=0$ and the estimate (5.36) of $J_{3}$ with the inequality (5.35) leads to

$$
\left\|\nabla \tilde{\chi}_{K}^{\xi}(x)-\nabla \chi^{\xi}\left(x_{K}, \frac{x}{\varepsilon}\right)\right\|_{L^{2}\left(K_{\delta}\right)} \leq C \sqrt{\left|K_{\Gamma}\right|}\left\|\chi^{\xi}\left(x_{K}, y\right)\right\|_{W^{1, \infty}(Y)} .
$$

Thus, $I_{1}$ can be estimated by the previous estimate and the Lipschitz continuity $\left(\mathcal{A}_{1}\right)$

$$
\left|I_{1}\right| \leq \frac{L}{\sqrt{\left|K_{\delta}\right|}}\left\|\nabla \tilde{\chi}_{K}^{\xi}(x)-\nabla \chi^{\xi}\left(x_{K}, \frac{x}{\varepsilon}\right)\right\|_{L^{2}\left(K_{\delta}\right)} \leq C\left(\frac{\varepsilon}{\delta}\right)^{1 / 2}\left\|\chi^{\xi}\left(x_{K}, y\right)\right\|_{W^{1, \infty}(Y)} .
$$

Combining the estimates (5.37) and (5.33) for $I_{1}$ and $I_{2}$, respectively, concludes the proof.

Proof of Theorem 4.6. The Theorem 4.6 is proved by combining the estimates from (5.31) (collocation error), (5.32) (periodic coupling) and Lemma 5.24 (Dirichlet coupling).

\section{IMPLEMENTATION AND NUMERICAL RESUltS}

In this section, we comment on the implementation of the multiscale method (3.3), illustrate the applicability to a test problem from material sciences and, for $p=2$ and $\alpha=1, \beta=2$ in $\left(\mathcal{A}_{1-2}\right)$, we give numerical studies of the convergence rates as well as the modeling error.

\subsection{Implementation}

In this section, we briefly discuss an implementation of the multiscale method (3.3). As the macroscopic equation (3.3) and the micro problems (3.6) are both nonlinear and coupled together, some care is needed. We thus describe how $u_{n+1}^{H} \in S_{0}^{1}\left(\Omega, \mathcal{T}_{H}\right)$ solving (3.3) is obtained for given $u_{n}^{H} \in S_{0}^{1}\left(\Omega, \mathcal{T}_{H}\right)$ and $n \in \mathbb{N}$.

At the macro level, the unknown $u_{n+1}^{H}$ is approximated by a sequence $\left\{u_{n+1}^{H,(j)}\right\}_{j \in \mathbb{N}}$ obtained by a Newton iteration for the macro equation (3.3) with the initial guess $u_{n+1}^{H,(0)}=u_{n}^{H}$. As the macro equation involves the nonlinear map $B^{H}$ given in (3.4), a set of constrained micro problems (3.6) has to be solved (at each macro iteration) and the Fréchet derivative of $B^{H}\left(v^{H} ; w^{H}\right)$ with respect to $v^{H}$ has to be computed. We follow the ideas from [31].

Newton's method for micro problems. Let $v^{H} \in S_{0}^{1}\left(\Omega, \mathcal{T}_{H}\right)$ be a macro function and $K \in \mathcal{T}_{H}$ with associated sampling domain $K_{\delta}$. The solution $v_{K}^{h}$ to the micro problem (3.6) is then computed by a Newton's 
method at microscopic level. In particular, for a given initial guess $v_{K}^{h,(0)}$, the micro solution $v_{K}^{h}$ is approximated by the sequence $\left\{v_{K}^{h,(j)}\right\}_{j \in \mathbb{N}}$ with $v_{K}^{h,(j+1)}-v^{H} \in S^{1}\left(K_{\delta}, \mathcal{T}_{h}\right)$ solving

$$
\mathcal{N}_{K}^{h}\left(v_{K}^{h,(j)} ; v_{K}^{h,(j+1)}-v_{K}^{h,(j)}, w^{h}\right)=-B_{K}^{h}\left(v_{K}^{h,(j)} ; w^{h}\right), \quad \forall w^{h} \in S^{1}\left(K_{\delta}, \mathcal{T}_{h}\right), j \in \mathbb{N},
$$

where the linear map $B_{K}^{h}\left(z^{H}+q^{h} ; \cdot\right)$ and the bilinear map $\mathcal{N}_{K}^{h}\left(z^{H}+q^{h} ; \cdot, \cdot\right)$ are given by

$$
\begin{aligned}
B_{K}^{h}\left(z^{H}+q^{h} ; w^{h}\right) & =\int_{K_{\delta}} \mathcal{A}^{\varepsilon}\left(x, \nabla z^{H}+\nabla q^{h}\right) \cdot \nabla w^{h} \mathrm{~d} x, \\
\mathcal{N}_{K}^{h}\left(z^{H}+q^{h} ; v^{h}, w^{h}\right) & =\int_{K_{\delta}} D_{\xi} \mathcal{A}^{\varepsilon}\left(x, \nabla z^{H}+\nabla q^{h}\right) \nabla v^{h} \cdot \nabla w^{h} \mathrm{~d} x,
\end{aligned}
$$

for $z^{H} \in S_{0}^{1}\left(\Omega, \mathcal{T}_{H}\right)$ and $q^{h}, v^{h}, w^{h} \in S^{1}\left(K_{\delta}, \mathcal{T}_{h}\right)$.

Further, the local contribution to the Fréchet derivative of $B^{H}$ is computed via an auxiliary micro problem, see [31]. For $z^{H} \in S_{0}^{1}\left(\Omega, \mathcal{T}_{H}\right)$ and $z_{K}^{h}$ its associated micro solution to (3.6), the auxiliary micro function $v_{K}^{h, z^{H}}$ solves: find $v_{K}^{h, z^{H}}-v^{H} \in S^{1}\left(K_{\delta}, \mathcal{T}_{h}\right)$ such that

$$
\mathcal{N}_{K}^{h}\left(z_{K}^{h} ; v_{K}^{h, z^{H}}, w^{h}\right)=0, \quad \forall w^{h} \in S^{1}\left(K_{\delta}, \mathcal{T}_{h}\right),
$$

where $\mathcal{N}_{K}^{h}$ is defined in (6.3). As the auxiliary micro problem (6.4) is linear it only leads to additional computational cost comparable to one iteration of the micro Newton's method (6.1).

Newton's method for macro scheme. For $j \in \mathbb{N}$, the $(j+1)$ th iterate $u_{n+1}^{H,(j+1)}$ of the macro Newton's method to approximate $u_{n+1}^{H}$ solves

$$
\begin{aligned}
\int_{\Omega} \frac{u_{n+1}^{H,(j+1)}-u_{n}^{H}}{\Delta t} w^{H} \mathrm{~d} x+\mathcal{N}^{H}\left(u_{n+1}^{H,(j)} ; u_{n+1}^{H,(j+1)}-u_{n+1}^{H,(j)}, w^{H}\right) \\
=\int_{\Omega} f w^{H} \mathrm{~d} x-B^{H}\left(u_{n+1}^{H,(j)} ; w^{H}\right), \quad \forall w^{H} \in S_{0}^{1}\left(\Omega, \mathcal{T}_{H}\right),
\end{aligned}
$$

where $B^{H}$ is given by (3.4) and $\mathcal{N}^{H}$ is defined for $v^{H}, w^{H}, z^{H} \in S_{0}^{1}\left(\Omega, \mathcal{T}_{H}\right)$ by

$$
\mathcal{N}^{H}\left(z^{H} ; v^{H}, w^{H}\right)=\sum_{K \in \mathcal{T}_{H}} \frac{|K|}{\left|K_{\delta}\right|} \int_{K_{\delta}} D_{\xi} \mathcal{A}^{\varepsilon}\left(x, \nabla z_{K}^{h}\right) \nabla v_{K}^{h, z^{H}} \mathrm{~d} x \cdot \nabla w^{H}\left(x_{K}\right),
$$

where $z_{K}^{h}$ is the micro solution to (3.6) associated to $z^{H}$ and $v_{K}^{h, z^{H}}$ is the solution to the auxiliary micro problem (6.4) constrained by $v^{H}$.

Remark 6.1. In practice, convergence up to machine precision is not needed for the micro Newton iterations. Instead, the stopping criterion for the micro Newton cycles should be adapted to the accuracy which is expected for the next macro iterate.

Further, if $p=2$ and $\alpha=1, \beta=2$ in $\left(\mathcal{A}_{0-2}\right)$, it holds that $D_{\xi} \mathcal{A}^{\varepsilon}$ is uniformly bounded and elliptic, see Remark 5.11. Thus, the problems (6.1) and (6.4) on the micro scale as well as the macro Newton iteration (6.5) admit a unique solution.

\subsection{Simulation of a laminated iron core}

In this section, we use the multiscale method (3.3) for a problem inspired by laminated iron cores. We refer to $[40,41]$ for multiscale simulations of the magnetostatics and magnetodynamics of such iron cores. 


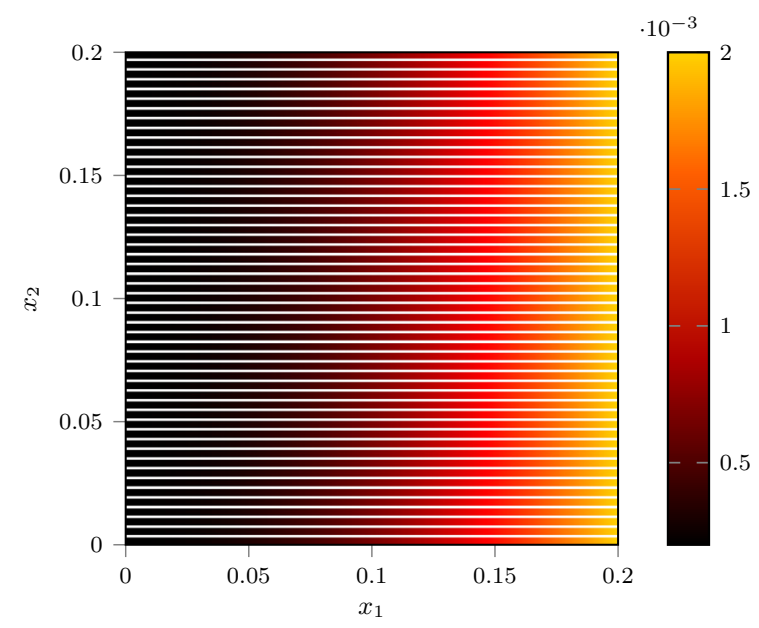

(a) $\mu^{\varepsilon}(x, \xi)$ for $\xi=(1 / 5,1 / 5)$. Insulation layers depicted as white areas.

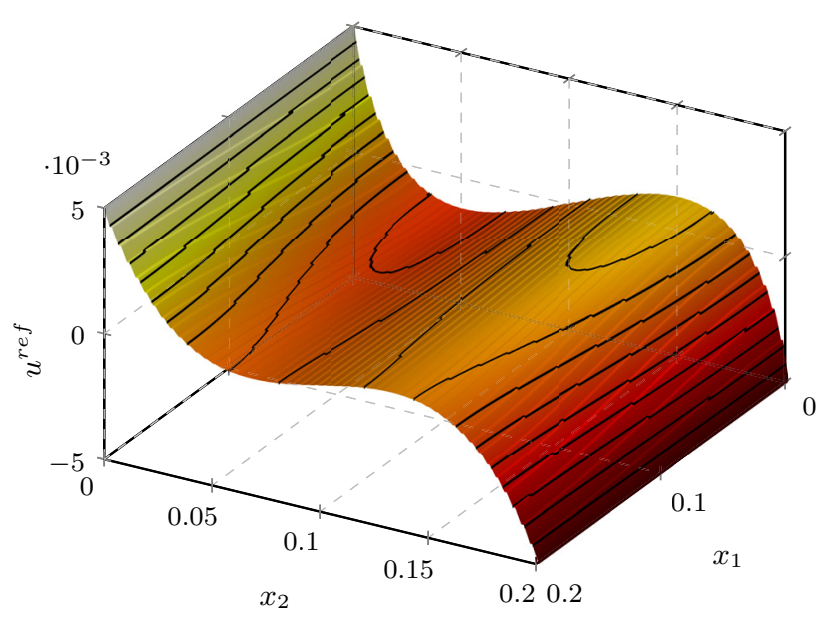

(b) Finescale solution at $T=2.10^{6}$ spatial degrees of freedom, 160 time steps.

FiguRE 1. Test problem with layered material of Section 6.2. Finescale solution obtained by standard FEM combined with implicit Euler method.

Setting. Let $\Omega=(0,0.2)^{2}$ and $T=2$. We consider a layered material modeled by the locally periodic map $\mathcal{A}^{\varepsilon}(x, \xi)=\mu^{\varepsilon}(x, \xi) \xi=\mu(x, x / \varepsilon, \xi) \xi$, where for $x=\left(x_{1}, x_{2}\right)^{T}, y=\left(y_{1}, y_{2}\right)^{T}$

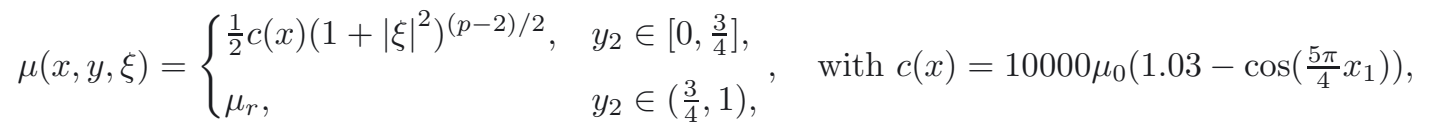

$p=1.03, \mu_{0}=4 \pi \times 10^{-7}, \mu_{r}=0.05$ and take $\varepsilon=1 / 5 \cdot(50+3 / 4)^{-1} \approx 0.0039$, see Figure 1a. Thus, the magnetic law is linear in the 50 insulation layers and nonlinear in the 51 lamination layers. The map $\mathcal{A}^{\varepsilon}$ is discontinuous in space and satisfies $\left(\mathcal{A}_{0-2}\right)$ for $p=1.03, \alpha=0.03, \beta=2$ (see [12], Rem. 2.1). We then solve (1.1) with instead of zero Dirichlet conditions - Dirichlet data $u_{D}\left(x_{1}, x_{2}, t\right)$ on $\Gamma_{D}=[0,0.2] \times\left\{x_{2}=0,0.2\right\}$, zero Neumann conditions on $\Gamma_{N}=\partial \Omega \backslash \Gamma_{D}$ and initial data $g\left(x_{1}, x_{2}\right)$, where

$$
u_{D}\left(x_{1}, 0.2, t\right)=\frac{1}{100}\left(\cos \left(\frac{\pi}{4} t\right)-\frac{1}{2}\right), u_{D}\left(x_{1}, 0, t\right)=-u_{D}\left(x_{1}, 0.2, t\right), \quad g\left(x_{1}, x_{2}\right)=\frac{1}{100}\left(5 x_{2}-\frac{1}{2}\right) .
$$

Reference solution. We compare the results obtained by the multiscale method (3.3) to a reference solution $u^{\text {ref }}$ calculated by standard FEM (on a mesh resolving the small scale $\varepsilon$ ) combined with the implicit Euler integrator (at 160 equidistant time steps), see Figure $1 \mathrm{~b}$ for $u^{\text {ref }}$ at final time $T=2$.

Numerical results. We use the multiscale method (3.3) on macro and micro meshes with $N_{\text {mac }}=32$ and $N_{\text {mic }} \in \mathbb{N} \backslash\{0\}$ elements, respectively, in each spatial dimension and $N=160$ equidistant time steps. For the upscaling, we collocate the data (6.6) in the slow variable $x$ and employ Dirichlet coupling $W\left(K_{\delta}\right)=H_{0}^{1}\left(K_{\delta}\right)$. As we use different values for $\delta \geq \varepsilon$, we adapt $N_{\text {mic }}$ such that $h \sim \delta / N_{\text {mic }}$ is constant.

In Table 1 we compare the FE-HMM solutions for $\delta=2^{k} \varepsilon, 0 \leq k \leq 5$, to $u^{\text {ref }}$ by calculating the error in the spatial $L^{2}$ norm (using $e_{\mathcal{C}^{0}\left(L^{2}\right)}$ defined in (6.11a)) and comparing the energy norms using $e_{\text {energy }}$ via

$$
\begin{array}{r}
e_{\text {energy }}=\left(\max _{0 \leq n \leq N}\left|\left\|u^{\mathrm{ref}}\left(\cdot, t_{n}\right)\right\|_{E}-\left\|u_{n}^{H}\right\|_{E}\right|\right)\left(\max _{0 \leq n \leq N}\left\|u^{\mathrm{ref}}\left(\cdot, t_{n}\right)\right\|_{E}\right)^{-1}, \quad \text { with } \\
\left\|u^{\mathrm{ref}}(\cdot, t)\right\|_{E}^{2}=\frac{1}{2}\left\|u^{\mathrm{ref}}(\cdot, t)\right\|_{L^{2}(\Omega)}^{2}+\int_{0}^{t} \int_{\Omega} \mathcal{A}^{\varepsilon}\left(x, \nabla u^{\mathrm{ref}}(x, \tau)\right) \cdot \nabla u^{\mathrm{ref}}(x, \tau) \mathrm{d} x \mathrm{~d} \tau, \\
\left\|u_{n}^{H}\right\|_{E}^{2}=\frac{1}{2}\left\|u_{n}^{H}\right\|_{L^{2}(\Omega)}^{2}+\sum_{k=0}^{\prime n} \Delta t \sum_{K \in \mathcal{T}_{H}}|K| \mathcal{A}_{K}^{0, h}\left(\nabla u_{k}^{H}\left(x_{K}\right)\right) \cdot \nabla u_{k}^{H}\left(x_{K}\right),
\end{array}
$$


TABLE 1. Comparison of the FE-HMM solutions to the standard FEM finescale solution $u^{\text {ref }}$ for the test problem of Section 6.2. Study of the influence of the size $\delta$ of the sampling domains $K_{\delta}$ for Dirichlet coupling. Error measured by $e_{\mathcal{C}^{0}\left(L^{2}\right)}$ and $e_{\text {energy }}$, see (6.11a) and (6.7), respectively.

\begin{tabular}{ccccccc}
\hline & $\delta=\varepsilon$ & $\delta=2 \varepsilon$ & $\delta=4 \varepsilon$ & $\delta=8 \varepsilon$ & $\delta=16 \varepsilon$ & $\delta=32 \varepsilon$ \\
\hline$e_{\mathcal{C}^{0}\left(L^{2}\right)}$ & 0.4832 & 0.3224 & 0.1971 & 0.1150 & 0.0682 & 0.0435 \\
\hline$e_{\text {energy }}$ & 0.2411 & 0.1274 & 0.0657 & 0.0334 & 0.0169 & 0.0083 \\
\hline
\end{tabular}

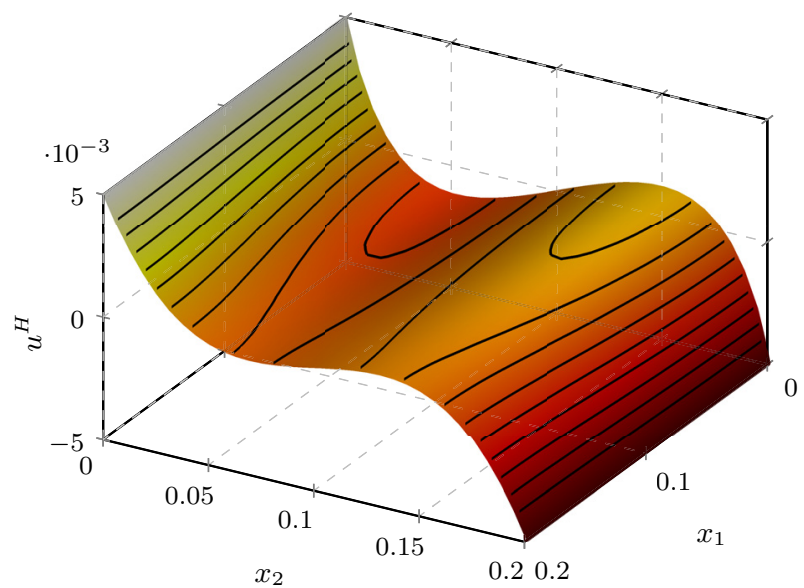

(a) FE-HMM with Dirichlet coupling, $\delta=32 \varepsilon$ and $N_{\text {mic }}=$ 1024.

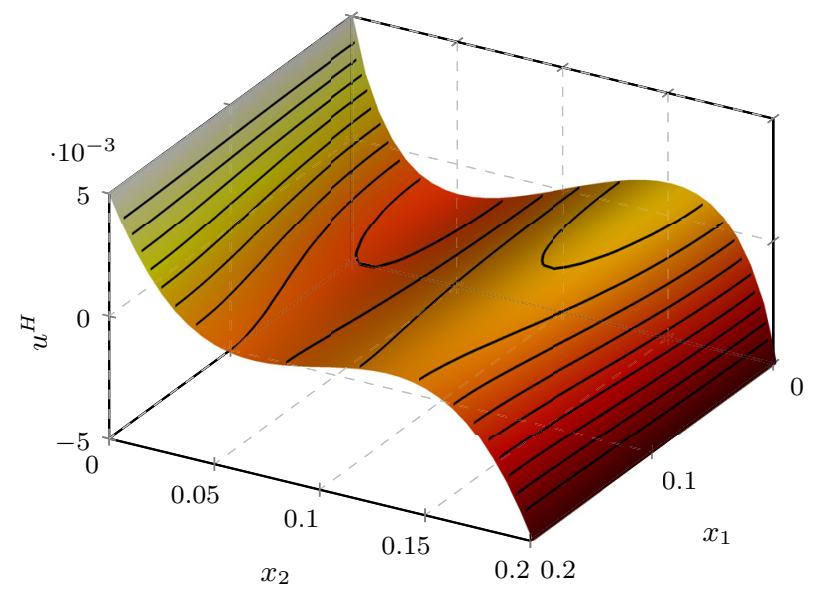

(b) FE-HMM with periodic coupling, $\delta=\varepsilon$ and $N_{\text {mic }}=32$.

Figure 2. Test problem with layered material of Section 6.2. FE-HMM solutions at final time $T=2$ computed with multiscale method (3.3) using Dirichlet or periodic coupling. Simulations with $N=160$ time steps, $N_{\text {mac }}=32$ macro elements per spatial dimension and constant micro error.

where $\sum^{\prime}$ indicates the trapezoidal rule in time. Note that for linear homogenization problems $e_{\mathcal{C}^{0}\left(L^{2}\right)}$ is known to be of order $\mathcal{O}(\varepsilon)$ and that the energy of $u^{\varepsilon}$ converges to the energy of $u^{0}$, while the error in the spatial $H^{1}$ norm is at least of order $\mathcal{O}(1)$. We observe that $e_{\mathcal{C}^{0}\left(L^{2}\right)}$ and $e_{\text {energy }}$ decrease monotonically when $\delta$ is increased $\left(e_{\text {energy }}\right.$ decays as $\left.\mathcal{O}\left(\delta^{-1}\right)\right)$, i.e., a sufficiently large $\delta$ is needed for reliable results.

Finally, we compare Dirichlet coupling with $\delta=32 \varepsilon$ to periodic coupling with parameter $\delta=\varepsilon$, see Figure 2 . While both solutions accurately capture the effective behavior of $u^{\text {ref }}$, the computation with periodic coupling and $\delta=\varepsilon$ needs much less micro degrees of freedom as the sampling domain is smaller.

Setting with non-periodic, random data. Let us next illustrate that the method (3.3) is applicable beyond the setting of (locally) periodic maps $\mathcal{A}^{\varepsilon}$. Therefore, we replace $c(x)$ in $(6.6)$ by the realization of a log-normal stochastic field (based on a normal distribution with zero mean and variance $\sigma^{2}=0.5$ ) with local correlation lengths $\varepsilon_{x_{1}}=0.002$ and $\varepsilon_{x_{2}}=0.004$ obtained by averaging via a moving ellipse (e.g., see [7], Sect. 4.2). Such data can be used, e.g., to model impurities in the ferromagnetic material.

First, analogously to Figure 1b, we compute a finescale reference solution $u^{\text {ref }}$ plotted in Figure 3a. Second, we apply the multiscale method (3.3) with parameters $N=160, N_{\text {mac }}=32, N_{\text {mic }}=256$ and Dirichlet coupling with $\delta=8 \varepsilon \approx 0.0315$, see Figure 3b. Comparing Figures $3 \mathrm{a}$ and $3 \mathrm{~b}$, we observe that the FE-HMM solution indeed reliably predicts the effective behavior of $u^{\text {ref }}$.

\subsection{Convergence rates}

We next validate the convergence rates (for $p=2$ and $\alpha=1, \beta=2$ in $\left(\mathcal{A}_{1-2}\right)$ ) stated in Section 4.2. 


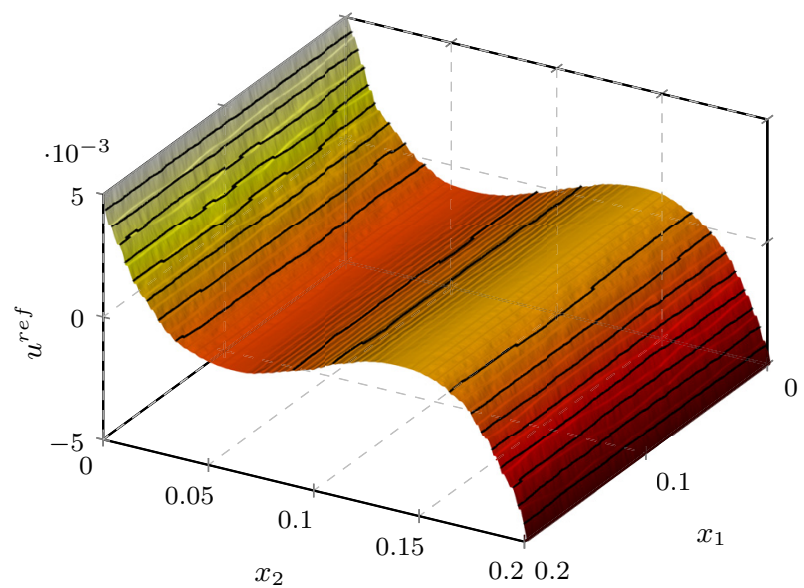

(a) Finescale solution $u^{\text {ref }} \cdot 10^{6}$ spatial degrees of freedom, 160 time steps.

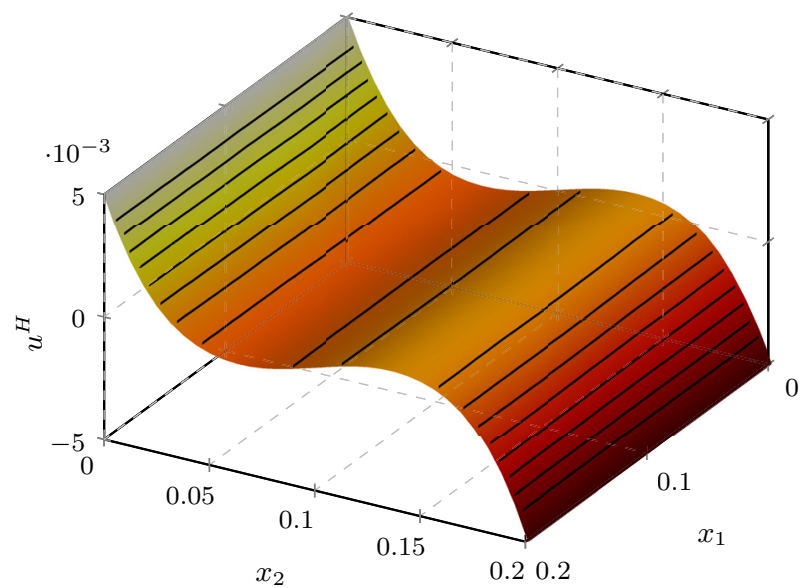

(b) FE-HMM solution. $N_{\text {mac }}=32, N_{\text {mic }}=256, N=160$ and Dirichlet coupling with $\delta=8 \varepsilon$.

FiguRE 3. Test problem with layered random material of Section 6.2. Reference solution (left) and FE-HMM solution computed with multiscale method (3.3) (right) at final time $T=2$.

Setting. For $\Omega=(0,1)^{2}$ and $T=2$, we consider (1.1) with maps $\mathcal{A}^{\varepsilon}$ and source $f$ chosen such that

$$
u^{0}(x, t)=\Phi(t)\left(x_{1}^{2}-x_{1}\right)\left(x_{2}^{2}-x_{2}\right), \quad \Phi(t)=21 \cdot\left(10 \cos \left(\frac{\pi}{2} t\right)+11\right)^{-1},
$$

is the homogenized solution $u^{0}$. Similar to Hoang [33], we construct a locally periodic map $\mathcal{A}^{\varepsilon}$ using the ansatz $\mathcal{A}^{\varepsilon}(t ; x, \xi)=\mathcal{A}_{p}(x / \varepsilon, \xi)+c(t ; x, x / \varepsilon)$ where $\mathcal{A}_{p}(\cdot, \xi)$ and $c(t ; x, \cdot)$ are $Y$-periodic. We then take

$$
\mathcal{A}_{p}(y, \xi)=\left[1+\sin \left(2 \pi\left(y_{1}+y_{2}\right)\right)+\left(\frac{9}{8}+\sin \left(2 \pi y_{1}+\frac{\pi}{3}\right)\right)\left(\frac{9}{8}+\cos \left(2 \pi y_{2}\right)\right)\left(1+|\xi|^{2}\right)^{-1 / 2}\right] \xi,
$$

which satisfies assumptions $\left(\mathcal{A}_{0-2}\right)$, and derive (using Maple) that

$$
f(x, t)=\Phi^{\prime}(t)\left(x_{1}^{2}-x_{1}\right)\left(x_{2}^{2}-x_{2}\right), \quad c(t ; x, y)=-\mathcal{A}_{p}\left(y,\left[e_{1}(t ; x, y), e_{2}(t ; x, y)\right]^{T}\right),
$$

where $\Phi^{\prime}(t)$ is the derivative of $\Phi(t)$ from (6.8) and $e_{i}(t ; x, y)$, for $i=1,2$, is given by

$$
e_{i}(t ; x, y)=\Phi(t)\left[\left(2 x_{i}-1\right)\left(x_{3-i}^{2}-x_{3-i}\right)+\left(x_{1}+x_{2}\right) \cos \left(2 \pi y_{i}\right) \sin \left(2 \pi y_{3-i}\right)\right] .
$$

Note that $\operatorname{div}(c(t ; x, x / \varepsilon))$ cannot be integrated into the source $f$, as it oscillates and grows as $\mathcal{O}\left(\varepsilon^{-1}\right)$. Further, the results of Section 4.2 can be extended to data $\mathcal{A}^{\varepsilon}, f$ (like (6.9)-(6.10)) smoothly varying in time.

Error measure. To measure the difference $u^{0}-u_{n}^{H}$, we use the relative error measures

$$
\begin{aligned}
& e_{\mathcal{C}^{0}\left(L^{2}\right)}=\max _{0 \leq n \leq N}\left(\sum_{K \in \mathcal{T}_{H}}\left\|u^{0}\left(\cdot, t_{n}\right)-u_{n}^{H}\right\|_{L^{2}(K)}^{2}\right)^{1 / 2}\left\|u^{0}\right\|_{\mathcal{C}^{0}\left([0, T], L^{2}(\Omega)\right)}^{-1}, \\
& e_{L^{2}\left(H^{1}\right)}=\left(\sum_{n=0}^{\prime} N t \sum_{K \in \mathcal{T}_{H}}\left\|\nabla u^{0}\left(\cdot, t_{n}\right)-\nabla u_{n}^{H}\right\|_{L^{2}(K)}^{2}\right)^{1 / 2}\left\|u^{0}\right\|_{L^{2}\left(0, T ; H_{0}^{1}(\Omega)\right)}^{-1},
\end{aligned}
$$

where $\sum^{\prime}$ indicates the trapezoidal rule in time and a high-order quadrature rule is used for $\|\cdot\|_{L^{2}(K)}$.

Numerical results. We use (3.3) on uniform triangular meshes on $\Omega$ and $K_{\delta}$ with $N_{\text {mac }}$ and $N_{\text {mic }}$ elements in each direction, respectively. Further, we choose periodic coupling with $\delta=\varepsilon=10^{-4}$ and collocate $\mathcal{A}(t ; x, x / \varepsilon, \xi)$ in $x$. Thus, the modeling error vanishes and we expect the convergence rates (4.7). 


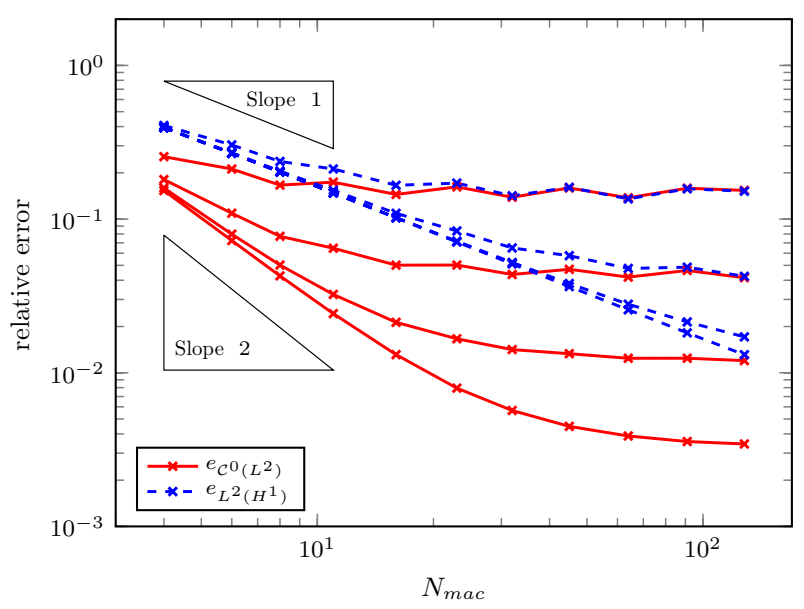

(a) Space discretization errors. The different lines correspond to a constant micro mesh $N_{\text {mic }}=4,8,16,32$. Number of time steps $N=2000$. Macro meshes with $N_{\text {mac }}=$ $4,6,8,11,16,23,32,45,64,91,128$.

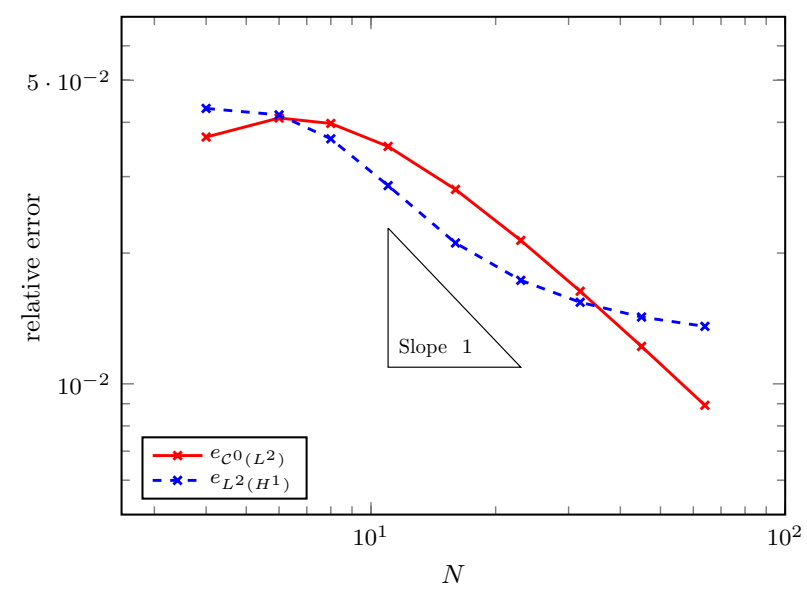

(b) Time discretization error. Macro and micro space discretization with constant meshes $N_{\text {mac }}=N_{\text {mic }}=128$. Number of time steps $N=4,6,8,11,16,23,32,45,64$.

Figure 4. Test problem of Section 6.3. Relative error measures $e_{\mathcal{C}^{0}\left(L^{2}\right)}$ (solid line) and $e_{L^{2}\left(H^{1}\right)}$ (dashed line), see (6.11), as a function of $N_{\text {mac }}$ (in part (a)) and $N$ (in part (b)), respectively.

To confirm the spatial convergence rates of Theorem 4.5, we plot in Figure 4a the measures $e_{\mathcal{C}^{0}\left(L^{2}\right)}$ and $e_{L^{2}\left(H^{1}\right)}$ versus $N_{\text {mac }} \sim 1 / H$ for a small $\Delta t=10^{-3}$. Indeed, for fixed $N_{\text {mic }}=4,8,16,32$, we get quadratic and linear convergence of $e_{\mathcal{C}^{0}\left(L^{2}\right)}$ and $e_{L^{2}\left(H^{1}\right)}$, respectively, for small $N_{\mathrm{mac}}$ and saturation levels for large $N_{\mathrm{mac}}$, which decrease by a factor around 4 when doubling $N_{\text {mic }}$ (quadratic micro convergence).

In Figure $4 \mathrm{~b}$ we plot the measures $e_{\mathcal{C}^{0}\left(L^{2}\right)}$ and $e_{L^{2}\left(H^{1}\right)}$ versus the number of time steps $N \sim 1 / \Delta t$ for fine spatial meshes with $N_{\text {mac }}=N_{\text {mic }}=128$. While $e_{L^{2}\left(H^{1}\right)}$ already saturates for $N>16$ (due to a large spatial macro error), we observe that $e_{\mathcal{C}^{0}\left(L^{2}\right)}$ converges linearly in $\Delta t$ as predicted by Theorem 4.5 .

\subsection{Influence of the sampling domain size $\delta$}

For $p=2, \alpha=1, \beta=2$ in $\left(\mathcal{A}_{1-2}\right)$, we next study the modeling error for Dirichlet and periodic coupling.

As in practice, the value of $\varepsilon$ is often not known, a common strategy is to use Dirichlet coupling (with $\delta$ larger than some available upper bound of $\varepsilon$ ) combined with oversampling techniques, e.g., see [30]. Interestingly, experimental studies however show that periodic coupling still performs well for general $\delta>\varepsilon$ (usually better than Dirichlet coupling), see $[5,7,51]$.

Setting. We modify the data of Section 6.3 by replacing $\Phi(t)$ and $\mathcal{A}_{p}$ in (6.8) and (6.9), respectively, by

$$
\Phi(x, t)=\cos \left(\frac{\pi}{2} t x_{2}\right), \quad \mathcal{A}_{p}(x, y, \xi)=\left[1+\left(2+\sin \left(2 \pi\left(y_{1}+y_{2}\right)\right)\right)\left(1+|\xi|^{2}\right)^{-1 / 2}\right] \xi,
$$

and compute then $c(t ; x, y)$ and $f(x, t)$ in (6.10) like in Section 6.3. This modification of the data allows a qualitatively better illustration of the effects.

Numerical results. We take $N=40$ time steps, $N_{\text {mac }}=32$ macro elements in each direction in $\Omega$, collocate $\mathcal{A}(x, x / \varepsilon, \xi)$ in $x$ and choose $\varepsilon=10^{-4}$. To keep the micro error constant for different $\delta$ we adapt the micro meshes such that $h \sim \delta / N_{\text {mic }}$ is constant (starting with $N_{\text {mac }}=N_{\text {mic }}$ for $\delta=\varepsilon$ ).

For Dirichlet coupling, we plot $e_{\mathcal{C}^{0}\left(L^{2}\right)}$ and $e_{L^{2}\left(H^{1}\right)}$ from (6.11) in Figure 5a versus $\delta / \varepsilon$ for $\delta_{i}=(10+i) / 10 \cdot \varepsilon$, $i=1, \ldots, 40$. We get an overall decrease with local peaks at $\delta / \varepsilon \in \mathbb{N}$ (resonance values). The envelopes for $e_{\mathcal{C}^{0}\left(L^{2}\right)}$ suggest a decay of $\mathcal{O}(\varepsilon / \delta)$ (like for linear problems, see [24]) rather than $\mathcal{O}(\sqrt{\varepsilon / \delta})$ as predicted in Theorem 4.6. The test problem however is a quasi $1 \mathrm{D}$ homogenization problem. 


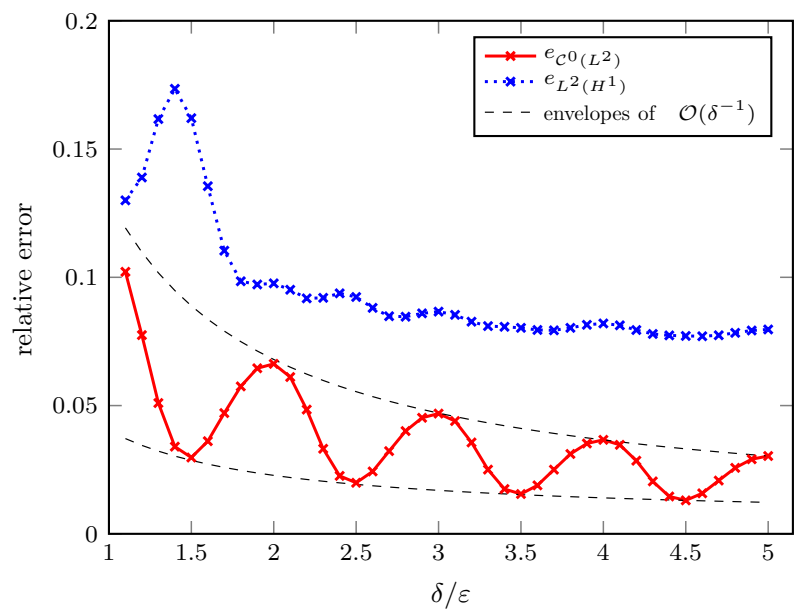

(a) Dirichlet coupling $W\left(K_{\delta}\right)=H_{0}^{1}\left(K_{\delta}\right)$ and $1.1 \leq \delta / \varepsilon \leq 5$.

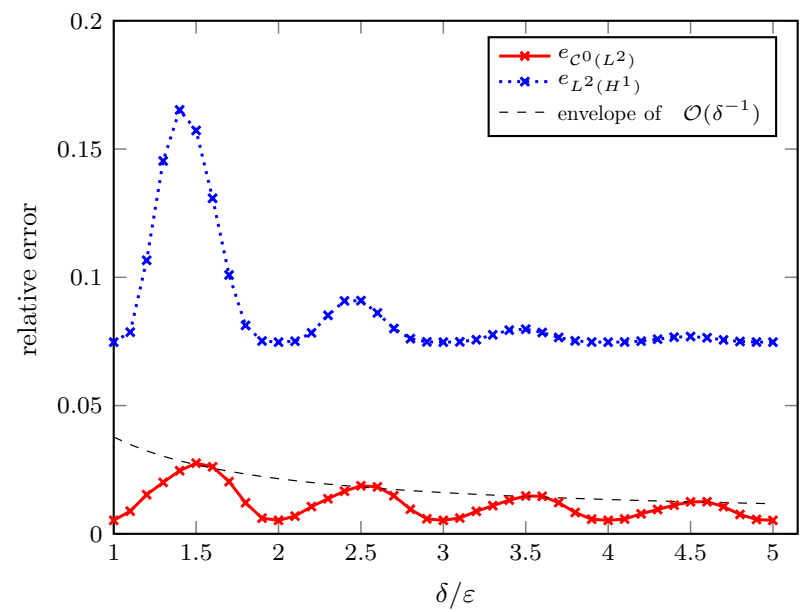

(b) Periodic coupling $W\left(K_{\delta}\right)=\mathcal{W}_{\text {per }}^{1,2}\left(K_{\delta}\right)$ and $1 \leq \delta / \varepsilon \leq 5$.

Figure 5. Test problem of Section 6.4. Relative error measures $e_{\mathcal{C}^{0}\left(L^{2}\right)}$ (solid line) and $e_{L^{2}\left(H^{1}\right)}$ (dotted line), see (6.11), as a function of the sampling domain size $\delta$. Constant number of time steps $N=40$ and $N_{\text {mac }}=32$ macro elements per spatial dimension. Microscopic mesh size $h$ chosen such that $h / \varepsilon=H$, i.e., remains constant for different sampling domain sizes $\delta$.

For periodic coupling and $\delta_{i}=(10+i) / 10 \cdot \varepsilon, i=0, \ldots, 40$, we discover in Figure $5 \mathrm{~b}$ a similar oscillating behavior coupled to a global decrease (again $\mathcal{O}\left(\delta^{-1}\right)$ for $e_{\mathcal{C}^{0}\left(L^{2}\right)}$ ). Optimal accuracy is obtained for $\delta / \varepsilon \in \mathbb{N}$ (see Thm. 4.6) and the local maxima at $\delta=(k+1 / 2) \varepsilon$ for $k \in \mathbb{N}$.

Finally, a fair comparison of Dirichlet and periodic coupling only makes sense if the overall error is essentially dictated by the modeling error (this can be checked using periodic coupling and $\delta / \varepsilon \in \mathbb{N}$, as then $r_{\bmod }=0$ ). While this is the case for $e_{\mathcal{C}^{0}\left(L^{2}\right)}$, the temporal and spatial errors, which are identical in all experiments, dominate for $e_{L^{2}\left(H^{1}\right)}$. Comparing then the measure $e_{\mathcal{C}^{0}\left(L^{2}\right)}$ reveals that periodic coupling is more accurate for all $\delta_{i}$. Similar results are known for linear homogenization problems, see [51].

\section{Conclusion}

We have proposed a multiscale method to solve nonlinear monotone parabolic homogenization problems by combining the implicit Euler integrator (in time) with a numerical homogenization procedure (based on the heterogeneous multiscale method) coupling macro and micro finite element simulations (in space).

First, we have proved in the general $L^{p}\left(W^{1, p}\right)$ setting, that the multiscale approximations converge towards the exact homogenized solution, for which only minimal regularity is assumed, if the effective model is wellapproximated by the upscaling strategy and the mesh sizes of the macro and micro spatial discretizations as well the time step size tend to zero. Second, in the $L^{2}\left(H^{1}\right)$ setting, we derived optimal a priori error estimates for the contributions of time and space discretization on macro and micro scale without any structural assumptions on the microscopic heterogeneities. Further, if we assume local periodicity of the data $\mathcal{A}^{\varepsilon}$, the modeling error has been explicitly estimated as well. We note that the error analysis can be generalized without any difficulties to different boundary conditions in (1.1) as well as maps $\mathcal{A}^{\varepsilon}$ and source terms $f$ smoothly varying in time.

Finally, we have shown that the computational cost of the multiscale method is independent of the small characteristic size of the micro structure. Thus, the method is well-suited for practical engineering problems if the quantity of interest involves the homogenized solution. However, the implementation of the proposed multiscale 
method still involves systems of nonlinear equations, see Section 6.1. As a remedy, a linearized variant of the FE-HMM is available in [10], see Section 1 for discussion.

Acknowledgements. This work was supported in part by the Swiss National Science Foundation under Grant 200021_134716/1.

\section{REFERENCES}

[1] A. Abdulle, On a priori error analysis of fully discrete heterogeneous multiscale FEM. Multiscale Model. Simul. 4 (2005) $447-459$.

[2] A. Abdulle, The finite element heterogeneous multiscale method: a computational strategy for multiscale PDEs, in Multiple scales problems in biomathematics, mechanics, physics and numerics. Vol. 31 of GAKUTO Internat. Ser. Math. Sci. Appl. Gakkōtosho, Tokyo (2009) 133-181.

[3] A. Abdulle, A priori and a posteriori error analysis for numerical homogenization: a unified framework. Ser. Contemp. Appl. Math. CAM 16 (2011) 280-305.

[4] A. Abdulle, W.E, B. Engquist and E. Vanden-Eijnden, The heterogeneous multiscale method. Acta Numer. 21 (2012) 1-87.

[5] A. Abdulle, M.J. Grote and C. Stohrer, Finite element heterogeneous multiscale method for the wave equation: long-time effects. Multiscale Model. Simul. 12 (2014) 1230-1257.

[6] A. Abdulle and M.E. Huber, Error estimates for finite element approximations of nonlinear monotone elliptic problems with application to numerical homogenization. Methods Partial Differ. Equ. 32 (2016) 955-969.

[7] A. Abdulle and A. Nonnenmacher, A short and versatile finite element multiscale code for homogenization problems. Comput. Methods Appl. Mech. Engrg. 198 (2009) 2839-2859.

[8] A. Abdulle and G. Vilmart, Coupling heterogeneous multiscale FEM with Runge-Kutta methods for parabolic homogenization problems: a fully discrete space-time analysis. Math. Models Methods Appl. Sci. 22 (2012) 1250002.

[9] A. Abdulle and G. Vilmart, Analysis of the finite element heterogeneous multiscale method for quasilinear elliptic homogenization problems. Math. Comp. 83 (2014) 513-536.

[10] A. Abdulle, M.E. Huber and G. Vilmart, Linearized numerical homogenization method for nonlinear monotone parabolic multiscale problems. Multiscale Model. Simul. 13 (2015) 916-952.

[11] J.W. Barrett and W.B. Liu, Finite element approximation of the parabolic p-Laplacian. SIAM J. Numer. Anal. 31 (1994) $413-428$.

[12] J.W. Barrett and W.B. Liu, Quasi-norm error bounds for the finite element approximation of a non-Newtonian flow. Numer. Math. 68 (1994) 437-456.

[13] A. Bensoussan, J.-L. Lions and G. Papanicolaou, Asymptotic analysis for periodic structures. North-Holland Publishing Co., Amsterdam (1978).

[14] S.C. Brenner and L.R. Scott, The mathematical theory of finite element methods. Vol. 15 of Texts Appl. Math., 3rd edn. Springer, New York (2008).

[15] V. Chiadò Piat, G. Dal Maso and A. Defranceschi, G-convergence of monotone operators. Ann. Inst. Henri Poincaré, Anal. Non Linéaire 7 (1990) 123-160.

[16] P.G. Ciarlet, The finite element method for elliptic problems. Vol. 4 of Stud. Math. Appl. North-Holland (1978).

[17] P.G. Ciarlet and P.A. Raviart, The combined effect of curved boundaries and numerical integration in isoparametric finite element methods, in The mathematical foundations of the finite element method with applications to partial differential equations (1972) 409-474.

[18] G. Dal Maso and A. Defranceschi, Correctors for the homogenization of monotone operators. Differ. Integral Equ. 3 (1990) $1151-1166$.

[19] J.E. Dendy Jr., Galerkin's method for some highly nonlinear problems. SIAM J. Numer. Anal. 14 (1977) $327-347$.

[20] L. Diening, C. Ebmeyer and M. Růžička, Optimal convergence for the implicit space-time discretization of parabolic systems with $p$-structure. SIAM J. Numer. Anal. 45 (2007) 457-472.

[21] M. Dobrowolski, $L^{\infty}$-convergence of linear finite element approximation to nonlinear parabolic problems. SIAM J. Numer. Anal. 17 (1980) 663-674.

[22] R. Du and P. Ming, Heterogeneous multiscale finite element method with novel numerical integration schemes. Commun. Math. Sci. 8 (2010) 863-885.

[23] W. E and B. Engquist, The heterogeneous multiscale methods. Commun. Math. Sci. 1 (2003) 87-132.

[24] W. E, P. Ming and P. Zhang, Analysis of the heterogeneous multiscale method for elliptic homogenization problems. J. Amer. Math. Soc. 18 (2005) 121-156.

[25] Y. Efendiev and A. Pankov, Numerical homogenization and correctors for nonlinear elliptic equations. SIAM J. Appl. Math. 65 (2004) 43-68.

[26] Y. Efendiev and A. Pankov, Numerical homogenization of nonlinear random parabolic operators. Multiscale Model. Simul. 2 (2004) 237-268.

[27] Y. Efendiev, T.Y. Hou and V. Ginting, Multiscale finite element methods for nonlinear problems and their applications. Commun. Math. Sci. 2 (2004) 553-589. 
[28] J. Frehse and R. Rannacher, Asymptotic $L^{\infty}$-error estimates for linear finite element approximations of quasilinear boundary value problems. SIAM J. Numer. Anal. 15 (1978) 418-431.

[29] A. Gloria, An analytical framework for the numerical homogenization of monotone elliptic operators and quasiconvex energies. Multiscale Model. Simul. 5 (2006) 996-1043.

[30] A. Gloria, Reduction of the resonance error. Part 1: Approximation of homogenized coefficients. Math. Models Methods Appl. Sci. 21 (2011) 1601-1630.

[31] P. Henning and M. Ohlberger, A Newton-scheme framework for multiscale methods for nonlinear elliptic homogenization problems, in Proc. of the ALGORITMY 2012, 19th Conference on Scientific Computing. Edited by Vysoké Tatry, Podbanské (2012) 65-74.

[32] P. Henning and M. Ohlberger, Error control and adaptivity for heterogeneous multiscale approximations of nonlinear monotone problems. Discrete Contin. Dyn. Syst. Ser. S 8 (2015) 119-150.

[33] V.H. Hoang, Sparse finite element method for periodic multiscale nonlinear monotone problems. Multiscale Model. Simul. 7 (2008) 1042-1072.

[34] V.H. Hoang and C. Schwab, High-dimensional finite elements for elliptic problems with multiple scales. Multiscale Model. Simul. 3 (2005) 168-194.

[35] P. Houston, J. Robson and E. Süli, Discontinuous Galerkin finite element approximation of quasilinear elliptic boundary value problems. I. The scalar case. IMA J. Numer. Anal. 25 (2005) 726-749.

[36] M.E. Huber, Numerical homogenization methods for advection-diffusion and nonlinear monotone problems with multiple scales. Ph.D. thesis, École Polytechnique Fédérale de Lausanne (2015).

[37] V.V. Jikov, S.M. Kozlov and O.A. Oleinik, Homogenization of differential operators and integral functionals. Springer-Verlag, Berlin, Heidelberg (1994).

[38] O.A. Ladyzhenskaya and N.N. Ural'tseva, Linear and quasilinear elliptic equations. Translated from the Russian by Scripta Technica. Inc. Translation editor: Leon Ehrenpreis. Academic Press, New York-London (1968).

[39] P. Ming and P. Zhang, Analysis of the heterogeneous multiscale method for parabolic homogenization problems. Math. Comp. 76 (2007) 153-177.

[40] I. Niyonzima, R.V. Sabariego, P. Dular and C. Geuzaine, Finite element computational homogenization of nonlinear multiscale materials in magnetostatics. IEEE Trans. Magn. 48 (2012) 587-590.

[41] I. Niyonzima, R.V. Sabariego, P. Dular, F. Henrotte and C. Geuzaine, Computational homogenization for laminated ferromagnetic cores in magnetodynamics. IEEE Trans. Magn. 49 (2013) 2049-2052.

[42] A. Pankov, G-convergence and homogenization of nonlinear partial differential operators. Vol. 422 of Math. Appl. Kluwer Academic Publishers, Dordrecht (1997).

[43] P.A. Raviart, The use of numerical integration in finite element methods for solving parabolic equations, in Topics in numerical analysis. Proc. of the Royal Irish Academy, Conference on Numerical Analysis (1972). Edited by J.J.H. Miller. Academic Press (1973) 233-264.

[44] N. Svanstedt, G-convergence of parabolic operators. Nonlinear Anal. 36 (1999) 807-842.

[45] N. Svanstedt, N. Wellander and J. Wyller, A numerical algorithm for nonlinear parabolic equations with highly oscillating coefficients. Numer. Methods Partial Differ. Equ. 12 (1996) 423-440.

[46] L. Tartar, Cours Peccot. Collège de France (1977).

[47] L. Tartar, The general theory of homogenization. A personalized introduction. Vol. 7 of Lect. Notes Unione Matematica Italiana. Springer-Verlag, Berlin, UMI, Bologna (2009).

[48] V. Thomée, Galerkin finite element methods for parabolic problems. Vol. 25 of Springer Ser. Comput. Math. 2nd edn. SpringerVerlag, Berlin (2006).

[49] M.F. Wheeler, A priori $L^{2}$ error estimates for Galerkin approximations to parabolic partial differential equations. SIAM J. Numer. Anal. 10 (1973) 723-759.

[50] J. Xu, Two-grid discretization techniques for linear and nonlinear PDEs. SIAM J. Numer. Anal. 33 (1996) $1759-1777$.

[51] X. Yue and W. E, The local microscale problem in the multiscale modeling of strongly heterogeneous media: effects of boundary conditions and cell size. J. Comput. Phys. 222 (2007) 556-572.

[52] E. Zeidler, Nonlinear functional analysis and its applications. II/A. Linear monotone operators, Translated from the German by the author and Leo F. Boron. Springer-Verlag, New York (1990).

[53] E. Zeidler, Nonlinear functional analysis and its applications. II/B. Nonlinear monotone operators, Translated from the German by the author and Leo F. Boron. Springer-Verlag, New York (1990). 